\title{
A Hi-GAL study of the high-mass star-forming region G29.96-0.02 ${ }^{\star}$
}

\author{
M. T. Beltrán ${ }^{1}$, L. Olmi ${ }^{1,2}$, R. Cesaroni ${ }^{1}$, E. Schisano ${ }^{3}$, D. Elia ${ }^{3}$, S. Molinari ${ }^{3}$, A. M. Di Giorgio ${ }^{3}$, J. M. Kirk ${ }^{4}$, \\ J. C. Mottram ${ }^{5}$, M. Pestalozzi ${ }^{3}$, L. Testi ${ }^{1,6}$, and M. A. Thompson ${ }^{7}$ \\ 1 INAF - Osservatorio Astrofisico di Arcetri, Largo E. Fermi 5, 50125 Firenze, Italy \\ e-mail: mbeltran@arcetri.astro.it \\ 2 University of Puerto Rico, Río Piedras Campus, Physics Dept., Box 23343, UPR station, San Juan, Puerto Rico, USA \\ 3 INAF - Istituto di Astrofisica e Planetologia Spaziali, via del Fosso del Cavaliere 100, 00133 Roma, Italy \\ 4 Jeremiah Horrocks Institute, University of Central Lancashire, Preston PR1 2HE, UK \\ 5 Leiden Observatory, Leiden University, PO Box 9513, 2300 RA Leiden, The Netherlands \\ ${ }^{6}$ ESO, Karl Schwarzschild str. 2, 85748 Garching, Germany \\ 7 Centre for Astrophysics Research, STRI, University of Hertfordshire, College Lane, Hatfield, AL10 9AB, UK
}

Received 11 January 2013 / Accepted 18 February 2013

\section{ABSTRACT}

\begin{abstract}
Context. G29.96-0.02 is a high-mass star-forming cloud observed at 70, 160, 250, 350, and $500 \mu \mathrm{m}$ as part of the Herschel survey of the Galactic plane (Hi-GAL) during the science demonstration phase.

Aims. We wish to conduct a far-infrared study of the sources associated with this star-forming region by estimating their physical properties and evolutionary stage, and investigating the clump mass function, the star formation efficiency and rate in the cloud.

Methods. We have identified the Hi-GAL sources associated with the cloud, searched for possible counterparts at centimeter and infrared wavelengths, fitted their spectral energy distribution and estimated their physical parameters.

Results. A total of 198 sources have been detected in all 5 Hi-GAL bands, 117 of which are associated with $24 \mu \mathrm{m}$ emission and 87 of which are not associated with $24 \mu \mathrm{m}$ emission. We called the former sources $24 \mu \mathrm{m}$-bright and the latter ones $24 \mu \mathrm{m}$-dark. The [70-160] color of the $24 \mu \mathrm{m}$-dark sources is smaller than that of the $24 \mu \mathrm{m}$-bright ones. The $24 \mu \mathrm{m}$-dark sources have lower $L_{\mathrm{bol}}$ and $L_{\mathrm{bol}} / M_{\mathrm{env}}$ than the $24 \mu \mathrm{m}$-bright ones for similar $M_{\mathrm{env}}$, which suggests that they are in an earlier evolutionary phase. The G29-SFR cloud is associated with 10 NVSS sources and with extended centimeter continuum emission well correlated with the $70 \mu \mathrm{m}$ emission. Most of the NVSS sources appear to be early B or late O-type stars. The most massive and luminous Hi-GAL sources in the cloud are located close to the G29-UC region, which suggests that there is a privileged area for massive star formation toward the center of the G29-SFR cloud. Almost all the Hi-GAL sources have masses well above the Jeans mass but only $5 \%$ have masses above the virial mass, which indicates that most of the sources are stable against gravitational collapse. The sources with $M_{\text {env }}>M_{\text {virial }}$ and that should be undergoing collapse and forming stars are preferentially located at $\lesssim 4^{\prime}$ of the G29-UC region, which is the most luminous source in the cloud. The overall SFE of the G29-SFR cloud ranges from 0.7 to 5\%, and the SFR ranges from 0.001 to $0.008 M_{\odot} \mathrm{yr}^{-1}$, consistent with the values estimated for Galactic HII regions. The mass spectrum of the sources with masses above $300 M_{\odot}$, well above the completeness limit, can be well-fitted with a power law of slope $\alpha=2.15 \pm 0.30$, consistent with the values obtained for the whole $l=30^{\circ}$, associated with high-mass star formation, and $l=59^{\circ}$, associated with low- to intermediate-mass star formation, Hi-GAL SDP fields.
\end{abstract}

Key words. ISM: individual objects: G29.96-0.02 - HII regions - stars: formation

\section{Introduction}

The G29.96-0.02 star-forming region (hereafter G29-SFR), located at a distance of $6.2 \mathrm{kpc}$ (Russeil et al. 2011), is a wellstudied high-mass star-forming cloud which falls in one of the two Science Demonstration Phase (SDP) fields observed by the ESA Herschel Space Observatory (Pilbratt et al. 2010) for the Herschel Infrared GALactic plane survey (Hi-GAL: Molinari et al. 2010). Hi-GAL is a Herschel key project aimed at mapping the Galactic plane in five photometric bands (70, 160, 250,350 , and $500 \mu \mathrm{m}$ ). Figure 1 shows the cloud as seen in different wavelengths, from 3.6 to $500 \mu \mathrm{m}$, by Spitzer and Herschel.

This cloud is dominated by IRAS $18434-0242$, the brightest source from 24 to $500 \mu \mathrm{m}$ (Fig. 1; Kirk et al. 2010), and one of the brightest radio and infrared sources in the Galaxy. This source is associated with a cometary UC HII region (hereafter G29-UC: Cesaroni et al. 1994; De Buizer et al. 2002) and with

* Tables 1-3 are available in electronic form at http: //WwW . aanda.org a hot molecular core (hereafter G29-HMC) located right in front of the cometary arc (Wood \& Churchwell 1989; Cesaroni et al. 1994, 1998). The G29-HMC core, which has been mapped in several tracers (Cesaroni et al. 1998; Pratap et al. 1999; Maxia et al. 2001; Olmi et al. 2003; Beuther et al. 2007; Beltrán et al. 2011), shows a velocity gradient approximately along the eastwest direction, which has been interpreted as rotation of a huge and massive toroid (4000 $\mathrm{AU}$ of radius and $88 M_{\odot}$ at a distance of $6.2 \mathrm{kpc}$ : Beltrán et al. 2011).

The G29-SFR cloud also contains a filament seen in absorption in the Spitzer images (Fig. 1) and in emission in the SCUBA Massive Pre-/Proto-cluster core Survey (SCAMPS: Thompson et al. 2005) at about $2^{\prime}$ east of the G29-UC region (see Spitzer image at $8 \mu \mathrm{m}$ in Fig. 1). This infrared dark cloud (IRDC) has been extensively studied at high-angular resolution in dust continuum emission and $\mathrm{NH}_{2} \mathrm{D}$ by Pillai et al. (2011), who have resolved, with an angular resolution better than $5^{\prime \prime}$, the dust and line emission of the filament into multiple massive cores with low temperatures, $<20 \mathrm{~K}$, and a high degree of deuteration. These findings support the idea that this massive IRDC is 


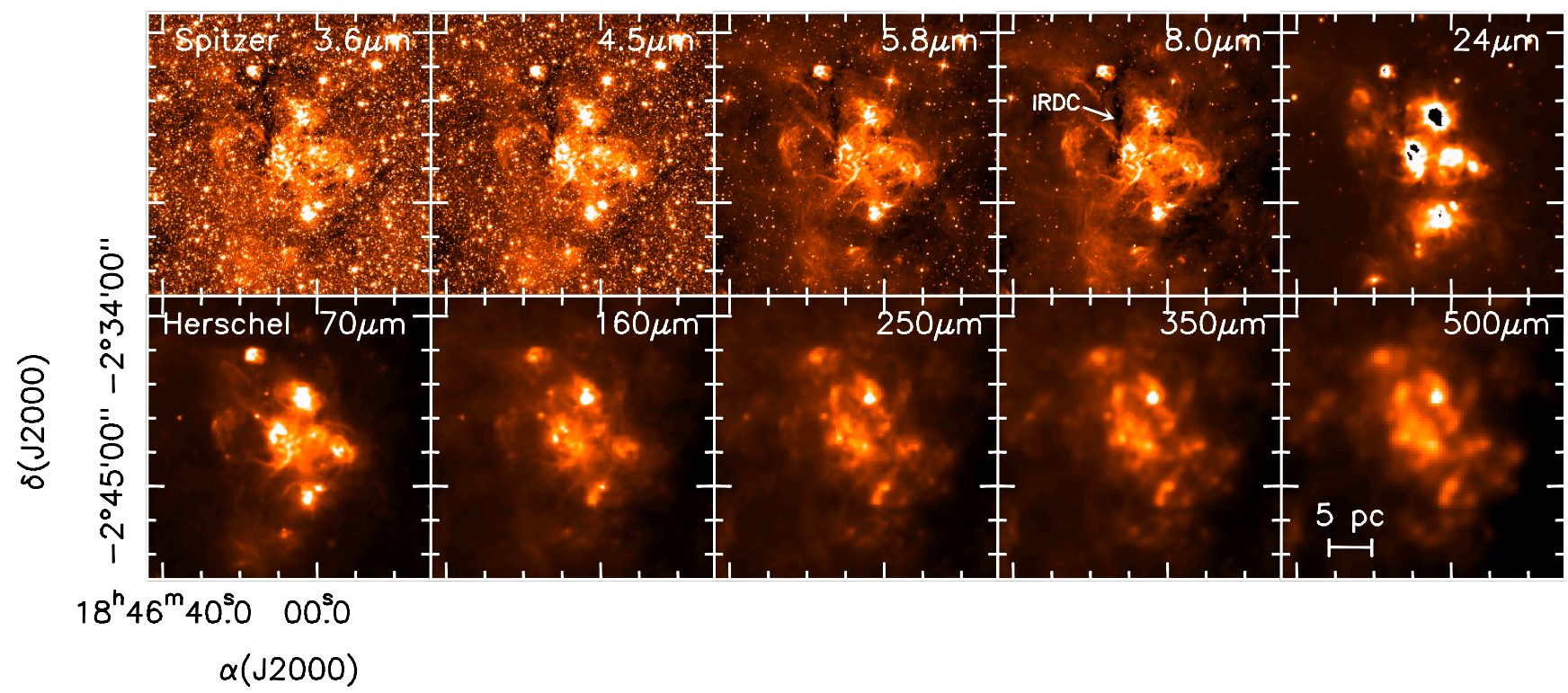

Fig. 1. Spitzer 3.6, 4.5, 5.8, 8.0, and $24 \mu \mathrm{m}$ and Herschel 70, 160, 250, 350, and $500 \mu \mathrm{m}$ images in linear scale of the G29-SFR cloud. The white arrow in the $8.0 \mu \mathrm{m}$ image points to the filamentary IRDC (Pillai et al. 2011).

in a very early stage of evolution, and could be in a pre-cluster phase. Only the brightest millimeter continuum core shows signs of high-mass star-formation activity, as indicated by the point source already visible at $24 \mu \mathrm{m}$ that is driving a molecular outflow. That no active star formation has been detected in other parts of this IRDC (Pillai et al. 2011) supports the idea of this extincted filament being in a very early evolutionary phase.

As just seen, the G29-SFR cloud represents an ideal laboratory to study star formation because young stellar objects in different evolutionary stages and different masses are embedded in it. In this paper, we present a far-infrared (FIR) study of this cloud using the Hi-GAL data in the 2 PACS and 3 SPIRE photometric bands, centered at 70, 160, 250, 350, and $500 \mu \mathrm{m}$. Our goal is to identify the FIR sources associated with this highmass star-forming region and estimate their physical properties (mass, temperature, luminosity, and density) together with the clump mass function (CMF) of the cloud. Combining the data with Spitzer and radio continuum observations, we will investigate the evolutionary stage of the sources and their distribution in the cloud, and the physical parameters of the associated HII regions. Finally, we will derive the star formation efficiency and star formation rate in this cloud. This work complements the other wide-field studies carried out as part of the Hi-GAL SDP (e.g. Bally et al. 2010; Battersby et al. 2011; Olmi et al. 2013).

\section{Source selection}

The first step to identify the Hi-GAL sources associated with the G29-SFR cloud is to define the limits of the molecular cloud. To study the distribution of the gas in the region we have used the ${ }^{13} \mathrm{CO}(1-0)$ data of the Boston University-Five College Radio Astronomy Observatory Galactic Ring Survey (GRS: Jackson et al. 2006). Toward the direction of the G29-UC region, the ${ }^{13} \mathrm{CO}(1-0)$ emission shows relatively narrow components at $\sim 8$, 49 , and $68 \mathrm{~km} \mathrm{~s}^{-1}$, and a much broader component from $\sim 90$ to $110 \mathrm{~km} \mathrm{~s}^{-1}$. Taking into account that the systemic velocity of high-density tracers, such as $\mathrm{NH}_{3}$ or $\mathrm{CH}_{3} \mathrm{CN}$, observed toward the G29-HMC core is $\sim 98-99 \mathrm{~km} \mathrm{~s}^{-1}$ (Cesaroni et al. 1998; Beltrán et al. 2011), we selected the latter broad velocity component to determine the distribution of the gas in the cloud. The ${ }^{13} \mathrm{CO}(1-0)$ emission has been averaged over the 95-105 $\mathrm{km} \mathrm{s}^{-1}$ velocity interval and compared with the HiGAL $250 \mu \mathrm{m}$ emission. As one can see in Fig. 2, the gas and dust emission are very well correlated. The G29-SFR cloud has been defined as the region contained approximately within the contour at $10-15 \%$ of the ${ }^{13} \mathrm{CO}$ peak emission $(5 \mathrm{~K})$ and that at $7 \%$ of the $250 \mu \mathrm{m}$ peak emission (36311 MJy/sr). Only the Hi-GAL sources falling inside this region have been assigned to the G29-SFR cloud.

\subsection{Source extraction}

The source extraction and brightness estimation techniques applied to the Hi-GAL maps in this work are similar to the methods used during analysis of the BLAST05 (Chapin et al. 2008) and BLAST06 data (Netterfield et al. 2009; Olmi et al. 2009). However, important modifications have been applied to adapt the technique to the SPIRE/PACS maps. The method used here defines in a consistent manner the region of emission of the same volume of gas/dust at different wavelengths, thus differing from the source grouping and band-merging procedures described by Molinari et al. (2011) and Elia et al. (2010). Candidate sources are identified by finding peaks after a Mexican hat wavelet type convolution is applied to all five SPIRE/PACS maps. Initial candidate lists from 70, 160 and $250 \mu \mathrm{m}$ are then found and fluxes at all three bands extracted by fitting a compact Gaussian profile to the source. Sources are not identified at 350 and $500 \mu \mathrm{m}$ due to the greater source-source and source-background confusion resulting from the lower resolution, and also because these two SPIRE wavebands are in general more distant from the peak of the source spectral energy distribution (SED). Each temporary source list at 70, 160 and $250 \mu \mathrm{m}$ is then purged of overlapping sources and then all three lists are merged. After selecting the sources based on their integrated flux and allowed angular diameter, a final source catalog is generated. In the next stage, Gaussian profiles are fitted again to all SPIRE/PACS maps, including the 350 and $500 \mu \mathrm{m}$ wavebands, using the size and location parameters determined at the shorter wavelengths during the previous steps (the size of the Gaussian is convolved to account for the differing beam sizes). Since the volume of emission is 


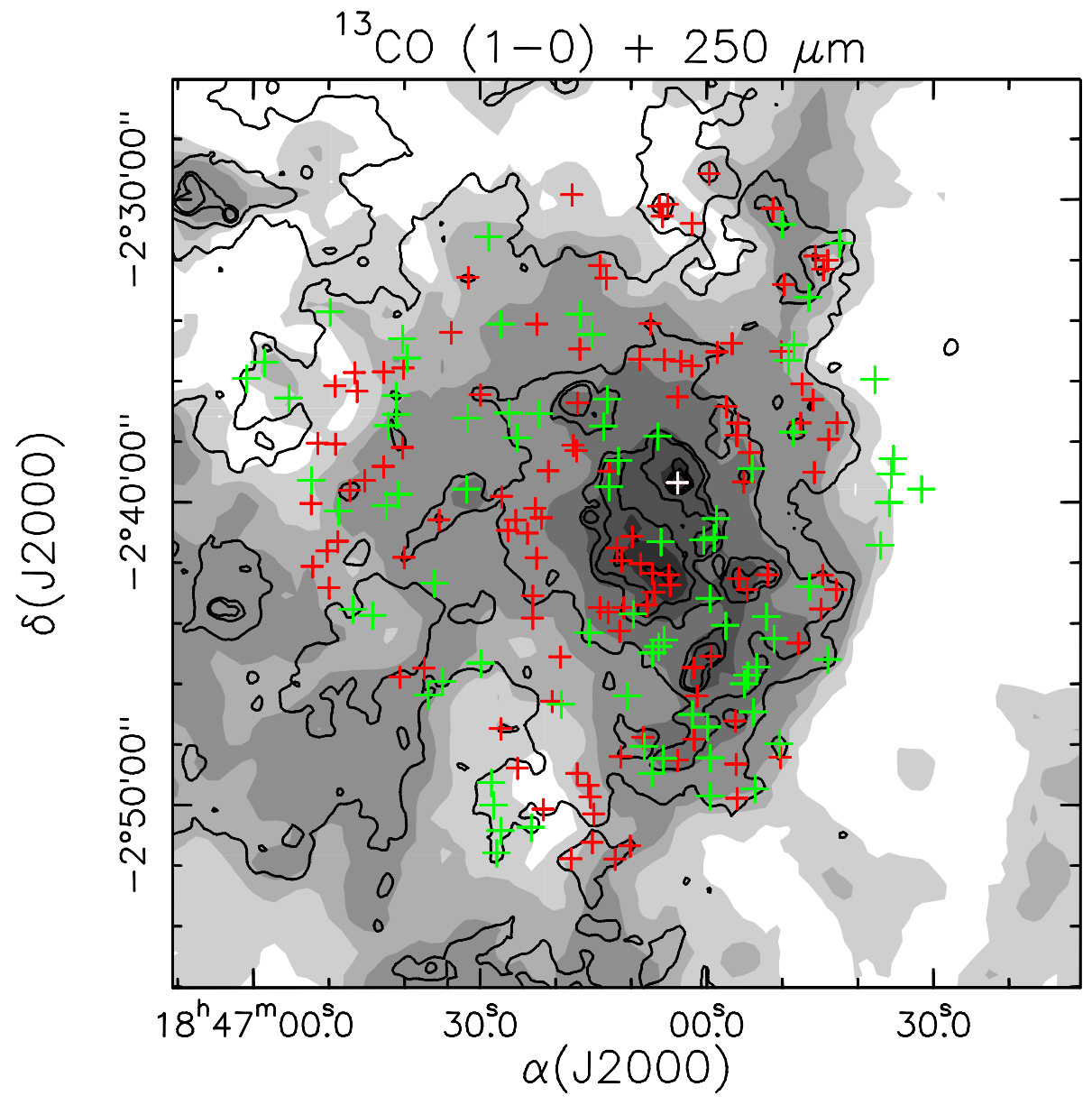

Fig. 2. Hi-GAL $250 \mu \mathrm{m}$ emission (contours) of the G29-SFR cloud overlaid on the ${ }^{13} \mathrm{CO}(1-0)$ emission (grayscale) from GRS averaged over the $95-105 \mathrm{~km} \mathrm{~s}^{-1}$ velocity interval. Contour levels are $7,9,14,18,24,36,48,72$, and $96 \%$ of the peak of the $250 \mu \mathrm{m}$ emission, $36311 \mathrm{MJy} / \mathrm{sr}$. Grayscale levels are 10, 15, $20,40,60,80$, and $100 \%$ of the peak of the ${ }^{13} \mathrm{CO}$ emission, $5 \mathrm{~K}$. The crosses indicate the positions of the Hi-GAL sources associated with the cloud detected in the 5 Hi-GAL photometric bands. Red and green crosses indicate, respectively, sources associated and nonassociated with Spitzer $24 \mu \mathrm{m}$ emission. The white cross marks the position of the Hi-GAL source associated with the G29-UC region and G29-HMC core (see Sect. 3.2), which is also associated with $24 \mu \mathrm{m}$ emission. basically defined using the $250 \mu \mathrm{m}$ band, this method does not fully exploit the higher angular resolution available at the shortest wavelengths. The interested reader can find more details in Olmi et al. (2013).

The total number of Hi-GAL sources associated with the G29-SFR cloud is 198. The position of the sources in equatorial and galactic coordinates, their fluxes in the 5 photometric Hi-GAL bands, and their possible association with MIPSGAL $24 \mu \mathrm{m}$ sources are given in Table 1 .

\section{Analysis}

\subsection{Spectral energy distribution fitting}

To estimate the dust temperature $T$, the mass $M_{\mathrm{env}}$, and the luminosity $L_{\text {bol }}$, of the sources associated with the G29-SFR cloud, we fitted their observed SED with a modified blackbody of the form $B_{v}(T)\left(1-\mathrm{e}^{-\tau_{v}}\right) \Omega_{\mathrm{S}}$, where $B_{v}(T)$ is the Planck function at a frequency $v$ for a dust temperature $T, \tau_{v}$ is the dust optical depth taken as $\tau_{v} \propto v^{\beta}$, where $\beta$ is the dust emissivity index, and $\Omega_{\mathrm{S}}$ is the source solid angle. The source size $\theta$, which is not deconvolved, was estimated at $160 \mu \mathrm{m}$ by the source extraction process (Olmi et al. 2013). The masses were calculated assuming a dust mass absorption coefficient of $0.5 \mathrm{~cm}^{2} / \mathrm{g}$ at $1.3 \mathrm{~mm}$ (Kramer et al. 2003) and a gas-to-dust ratio of 100 . To check whether the SED fitting improved, we searched for counterparts of the Hi-GAL sources at shorter wavelengths in the MIPSGAL $24 \mu \mathrm{m}$ catalog (Shenoy et al. 2012). The method used to associate Hi-GAL and MIPSGAL sources, which was based on both a positional and a color criteria, is described by Olmi et al. (2013). For the remaining Hi-GAL sources or those sources saturated at $24 \mu \mathrm{m}$, we searched for a counterpart in the Wide-field Infrared Survey Explorer (WISE) catalog at $22 \mu \mathrm{m}$ (Wright et al. 2010). To associate a WISE source to a Hi-GAL source, we arbitrarily chose the closest WISE source located at $<12^{\prime \prime}$, the WISE angular resolution at $22 \mu \mathrm{m}$ (Wright et al. 2010). Finally, for the remaining Hi-GAL sources or those sources saturated at $22 \mu \mathrm{m}$, we searched for a counterpart in the Midcourse Space Experiment (MSX) catalog at $21 \mu \mathrm{m}$ (Price et al. 2001). In this case, we arbitrarily associated the closest MSX source located at $<18^{\prime \prime} .3$, the MSX angular resolution at $21 \mu \mathrm{m}$ (Price et al. 2001). We found 103 MIPSGAL sources not saturated at $24 \mu \mathrm{m}, 11 \mathrm{WISE}$ sources not saturated at $22 \mu \mathrm{m}$, and 6 MSX sources associated with the Hi-GAL ones. The SED fitting was performed using the $5 \mathrm{Hi}-\mathrm{GAL}$ bands for 157 sources. For these 157 sources having a counterpart at shorter wavelengths, including the additional point in the SED did not improve the fitting. For 13 sources, only the $160,250,350$, and $500 \mu \mathrm{m} \mathrm{Hi-GAL}$ bands were used. For these sources, the flux at $160 \mu \mathrm{m}, S_{160 \mu \mathrm{m}}$, was $\leq S_{250 \mu \mathrm{m}}$ and including the $S_{70 \mu \mathrm{m}}$ in the SED clearly worsen the fit. This indicates that the $70 \mu \mathrm{m}$ emission is likely tracing a different source component, more associated with the central stellar object, than that traced by the emission at 160 to $500 \mu \mathrm{m}$, more associated with the extended envelope surrounding the central source. The $5 \mathrm{Hi}-\mathrm{GAL}$ bands plus the $21 \mu \mathrm{m}$ band of MSX were used in the SED fitting for 4 sources. In these cases, $S_{70 \mu \mathrm{m}}>S_{160 \mu \mathrm{m}}$ and including the flux at $21 \mu \mathrm{m}$, which is smaller than that at $70 \mu \mathrm{m}$, clearly improved the fitting. For 6 sources, the 5 Hi-GAL bands plus the $22 \mu \mathrm{m}$ band of the WISE were used in the fitting. For these sources, $S_{70 \mu \mathrm{m}}>S_{160 \mu \mathrm{m}}$ and $S_{70 \mu \mathrm{m}}>S_{22 \mu \mathrm{m}}$, and again, including the flux at a shorter wavelength improved the fitting. 


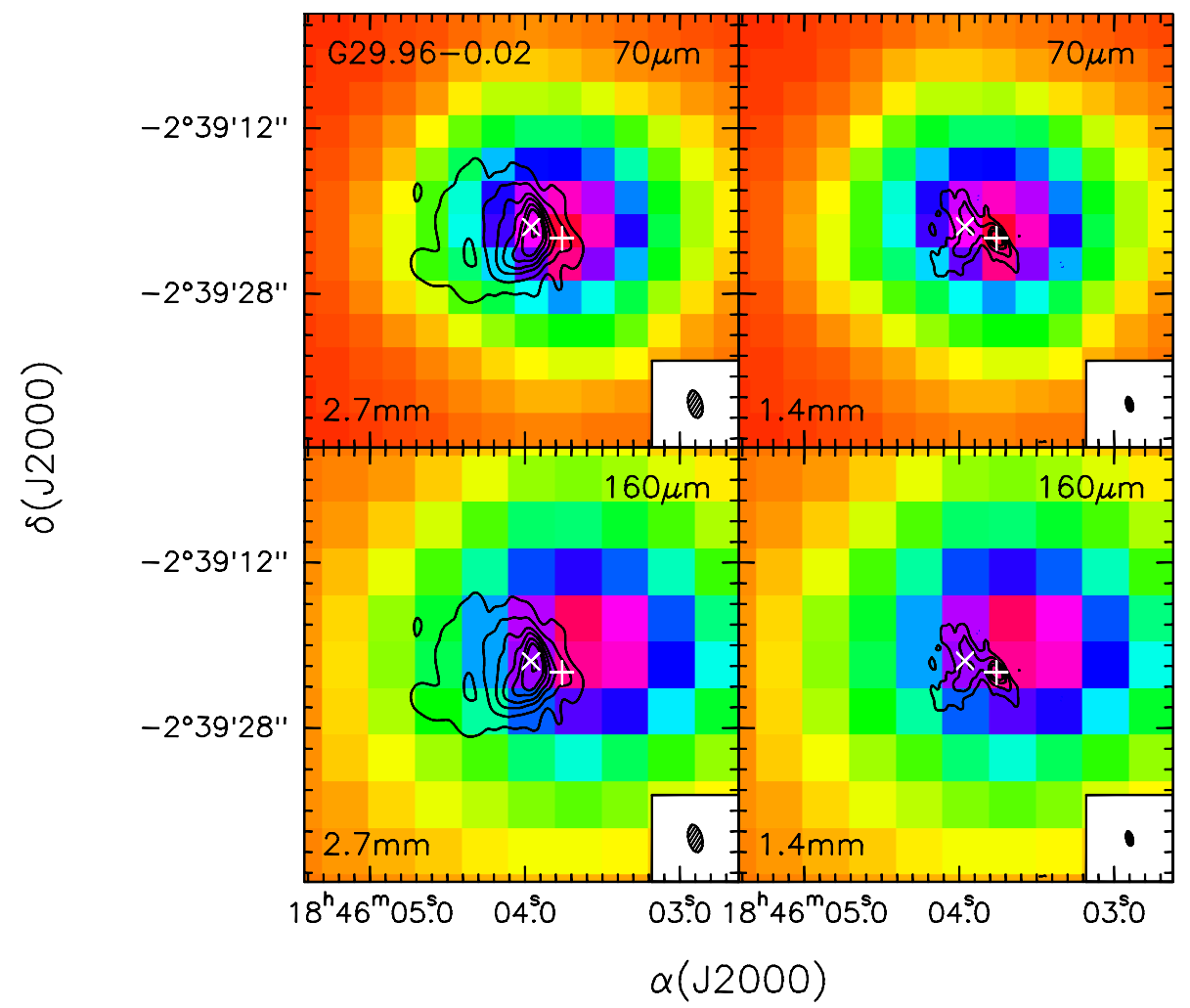

Fig. 3. Overlay of the $2.7 \mathrm{~mm}$ (left panels) and the $1.4 \mathrm{~mm}$ (right panels) continuum emission (contours) obtained with the PdBI (Beltrán et al. 2011) on the Hi-GAL $70 \mu \mathrm{m}$ (upper panels) and $160 \mu \mathrm{m}$ (lower panels) emission (grayscale) toward the position of the G29$\mathrm{UC}$ region and the G29-HMC core. The white $\mathrm{X}$ marks the position of the G29-UC region (Wood \& Churchwell 1989) and the white cross the position of the $1.4 \mathrm{~mm}$ continuum emission peak associated with the G29-HMC core (Beltrán et al. 2011). The contour levels are 3, $9,18,27,39,51$, and 75 times $3 \mathrm{mJy}^{-1}$ at $2.7 \mathrm{~mm}^{2}$ and $6.7 \mathrm{mJy}^{-1}$ beam ${ }^{-1}$ at $1.4 \mathrm{~mm}$. The PdBI synthesized beam is shown in the lower righthand corner.
Finally, for 18 sources, the $5 \mathrm{Hi}-\mathrm{GAL}$ bands plus the $24 \mu \mathrm{m}$ MIPSGAL band were used. For these sources, $S_{70 \mu \mathrm{m}}>S_{160 \mu \mathrm{m}}$ and $S_{70 \mu \mathrm{m}}>S_{24 \mu \mathrm{m}}$, and as in the previous cases, including the flux at a shorter wavelength improved the fitting. The MSX flux at $21 \mu \mathrm{m}$, the WISE flux at $22 \mu \mathrm{m}$, and the MIPSGAL flux at $24 \mu \mathrm{m}$ used for the SED fitting of these 28 sources is given in Table 2. Table 3 shows the values of $\theta$, obtained from the source extraction process, of $\beta, T, M_{\mathrm{env}}$, and $L_{\mathrm{bol}}$, obtained from the SED fitting, and of the surface density $\Sigma$, for the 198 sources. The surface density was calculated following the expression $\Sigma=M_{\mathrm{env}} /\left(\pi \times R^{2}\right)$, where the radius of the sources $R$ was obtained from their sizes, $\theta$, and following the expression $R=\theta / 2 \times d$, where $d$ is the distance to the G29-SFR cloud.

\subsection{The Hi-GAL source associated with the G29-UC region and G29-HMC core}

Figure 3 shows an overlay of the continuum emission at 2.7 and $1.4 \mathrm{~mm}$ obtained with the IRAM-Plateau de Bure interferometer (PdBI) (Beltrán et al. 2011) on the Hi-GAL maps at 70 and $160 \mu \mathrm{m}$ toward the position of the G29-UC region and G29-HMC core. The Hi-GAL source in our catalog is \#242. As seen in Table 1, this is the brightest source in all 5 Hi-GAL bands. At 2.7 and $1.4 \mathrm{~mm}$, the G29-UC region is outlined by continuum emission showing a cometary arc shape, while the G29-HMC core emission is visible westwards in front of the arc. The emission of the G29-HMC core is better resolved at $1.4 \mathrm{~mm}$, where it shows a flattened structure. The peak of the $1.4 \mathrm{~mm}$ continuum emission (Beltrán et al. 2011), indicated with a white cross in Fig. 3, coincides with the G29-HMC core. As one can see in this figure, at $70 \mu \mathrm{m}$, the emission seems to be mainly associated with the G29-HMC core. In fact, the peak of the $70 \mu \mathrm{m}$ emission coincides with that of the $1.4 \mathrm{~mm}$ continuum emission. At $160 \mu \mathrm{m}$, the emission also seems to be more associated with the HMC than with the UC HII region, although in this case the

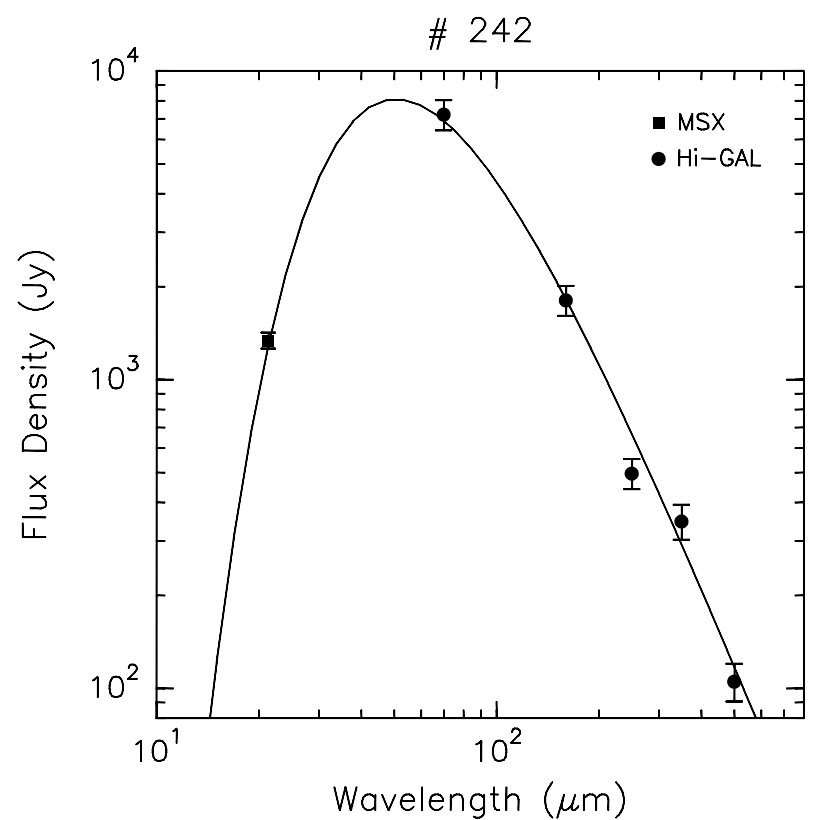

Fig. 4. SED of the Hi-GAL source \#242, associated with the G29-UC region and G29-HMC core. The black circles and black square show the Hi-GAL and MSX at $21 \mu \mathrm{m}$ data, respectively, with error bars. The black solid line represents the best-fit modified blackbody.

peak of the Hi-GAL emission is located toward the north of the G29-HMC core. The angular resolution of the Hi-GAL emission at 250,350 , and $500 \mu \mathrm{m}$ is not enough to properly study with which component, the HMC or the UC HII region, this submillimeter emission is associated.

From the SED fitting (Fig. 4), we derived a mass of $\sim 2880 M_{\odot}$ for source \#242, for a dust temperature of $77 \mathrm{~K}$, the highest of the sources in the G29-SFR cloud, a size of $\sim 19^{\prime \prime}$, and 
Table 4. Mean (median) physical parameters of the Hi-GAL sources.

\begin{tabular}{lccc}
\hline \hline & All sources & $24 \mu$ m-dark & $24 \mu$ m-bright \\
\hline Radius $(\mathrm{pc})$ & $0.36(0.36)$ & $0.34(0.35)$ & $0.37(0.38)$ \\
Mass $\left(M_{\odot}\right)$ & $379(115)$ & $435(172)$ & $340(86)$ \\
Surface density $\left(\mathrm{g} \mathrm{cm}^{-2}\right)$ & $0.24(0.06)$ & $0.27(0.1)$ & $0.22(0.04)$ \\
Temperature $(\mathrm{K})$ & $29(25)$ & $22(22)$ & $33(30)$ \\
Luminosity $\left(L_{\odot}\right)$ & $6.2 \times 10^{3}(470)$ & $706(247)$ & $1 \times 10^{4}(713)$ \\
Luminosity-to-mass ratio $\left(L_{\odot} / M_{\odot}\right)$ & $23(5)$ & $6(2)$ & $34(10)$ \\
\hline
\end{tabular}

a dust emissivity index of 0.8 . The surface density is $2.3 \mathrm{~g} \mathrm{~cm}^{-2}$, well above the theoretical threshold of $1 \mathrm{~g} \mathrm{~cm}^{-2}$ (Krumholz \& McKee 2008) necessary for high-mass star formation to occur. The luminosity of this source is $\sim 8 \times 10^{5} L_{\odot}$ and is the highest in the whole cloud. Kirk et al. (2010) constructed the SED of this source by using the SPIRE Fourier transform spectrometer data from 190 to $670 \mu \mathrm{m}$ and archival data from 2.4 to $1.3 \mathrm{~mm}$ (see their Fig. 1). From the SED fitting, these authors obtained a temperature of $\sim 80 \mathrm{~K}$, in agreement with our value, and a dust emissivity index of $\sim 1.7$, twice the one that we obtained. The dust luminosity integrated under the fitted modified blackbody in the range $2-2000 \mu \mathrm{m}$ is $1.6 \times 10^{6} L_{\odot}$, assuming a distance of $8.9 \mathrm{kpc}$. The luminosity would be $\sim 8 \times 10^{5} L_{\odot}$ for a distance of $6.2 \mathrm{kpc}$, in agreement with our estimated $L_{\mathrm{bol}}$. As for the mass, Kirk et al. (2010) estimate a mass of $1500 M_{\odot}$, assuming a distance of $8.9 \mathrm{kpc}$, using the fitted dust temperature and the SCUBA $850 \mu$ m flux density (Thompson et al. 2006). The mass would be $\sim 730 M_{\odot}$ for a distance of $6.2 \mathrm{kpc}$. This value is a factor $\sim 4$ smaller than the one that we obtained from the SED fitting. Besides the different method used to estimate the mass, this difference could be accounted for, in part, by the different opacity coefficient $\left(0.01 \mathrm{~cm}^{2} / \mathrm{g}\right.$ at $\left.850 \mu \mathrm{m}\right)$ and dust emissivity index $(\beta=1.7)$ used by these authors.

\subsection{Source physical parameters}

Figure 5 shows the distribution of radii, masses, surface densities, temperatures, luminosities and luminosity-to-mass ratios of the sources. Table 4 shows the mean and median values for the same physical quantities. Beltrán et al. (2006) observed a sample of southern hemisphere high-mass protostellar candidates at $1.2 \mathrm{~mm}$ with the SEST antenna. In the following we will confront the physical parameters obtained for the sources in the G29-SFR cloud with those of Beltrán et al. (2006) because them carried out a detailed comparison of the values of their sources with those estimated in other millimeter continuum surveys. The mean and median values of $0.36 \mathrm{pc}$ for the radius of the Hi-GAL sources associated with the G29-SFR cloud suggest that these sources are probably clumps (e.g. Giannini et al. 2012) that will not form individual stars but multiple star systems or star clusters. Unfortunately, the Herschel observations do not have enough spatial resolution to resolve these clumps into individual cores or stars. These values of the radius are consistent with the mean and median values of 0.25 and $0.2 \mathrm{pc}$ found by Beltrán et al. (2006). The mean and median values of the mass are also consistent with the mean and median values of 320 and $102 M_{\odot}$ found by Beltrán et al. (2006) for their sample, and indicates that the sources associated with the G29-SFR cloud and detected by Herschel are mostly massive objects. The mean temperature is in agreement with the mean temperature of $28 \mathrm{~K}$ found by Beltrán et al. (2006), and with the value of $32 \mathrm{~K}$ found by Molinari et al. (2000) for a sample of luminous high-mass protostellar candidates in the northern hemisphere.
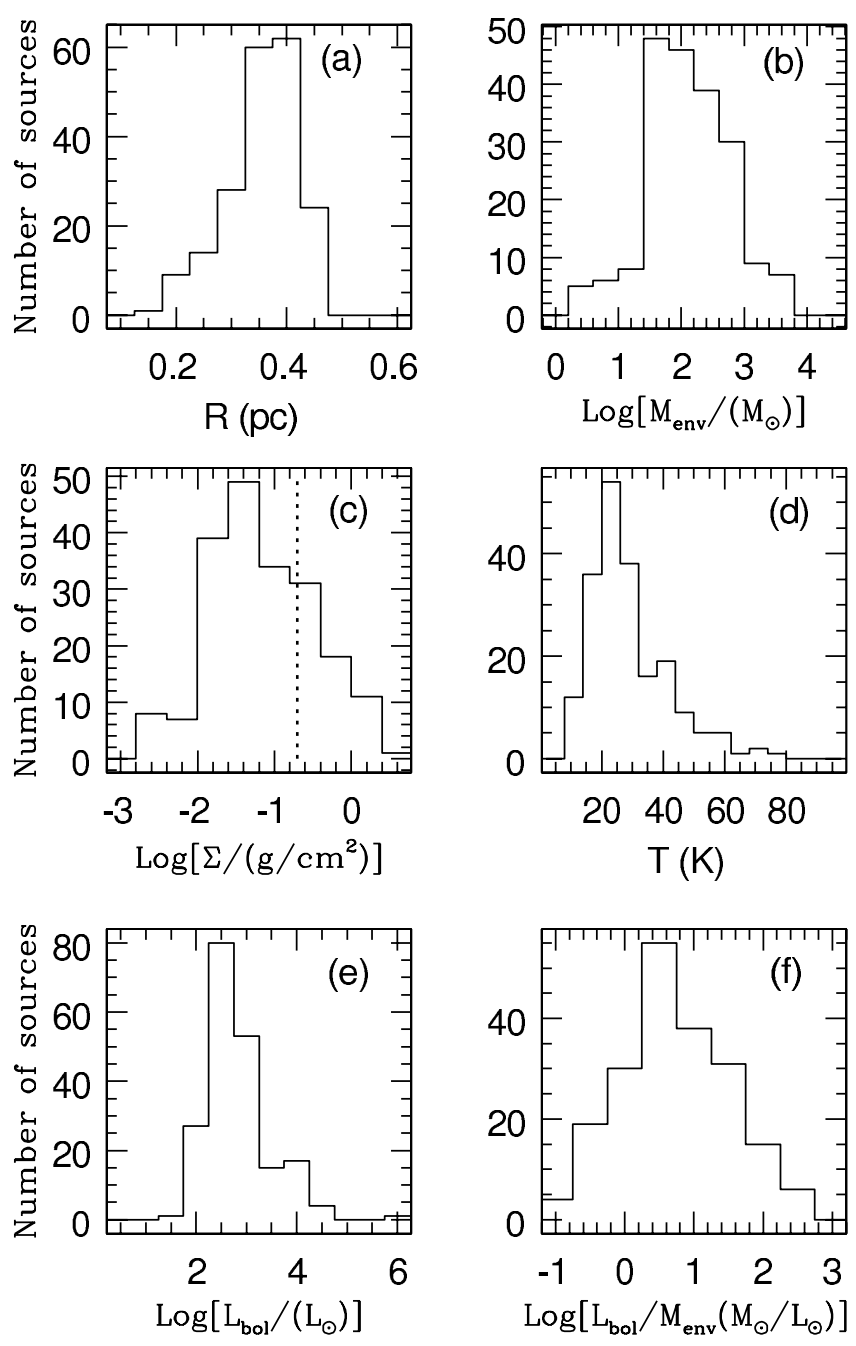

Fig. 5. Histograms of some parameters of the Hi-GAL sources detected toward G29: a) radius of the sources; b) mass; c) $\mathrm{H}_{2}$ surface density; d) temperature; e) luminosity; and f) luminosity-to-mass ratio. The dotted line in panel $c$ indicates the minimum surface density needed to form massive stars according to theory (Butler \& Tan 2012).

The average and median values of the surface density, of 0.24 and $0.06 \mathrm{~g} \mathrm{~cm}^{-2}$, are similar to the mean and median values of 0.4 and $0.14 \mathrm{~g} \mathrm{~cm}^{-2}$ estimated by Beltrán et al. (2006). These values are slightly lower than the minimum surface density needed, according to theory (Krumholz \& McKee 2008), to form massive stars. In a recent work, Butler \& Tan (2012) find typical mass surface densities of $0.15 \mathrm{~g} \mathrm{~cm}^{-2}$ for cores, and of $0.3 \mathrm{~g} \mathrm{~cm}^{-2}$ for clumps in infrared dark clouds, some of which are likely to form massive stars. Butler \& Tan (2012) consider the cores as structures of about $100 M_{\odot}$ embedded in clumps. These cores, which are virialized and in approximate pressure 


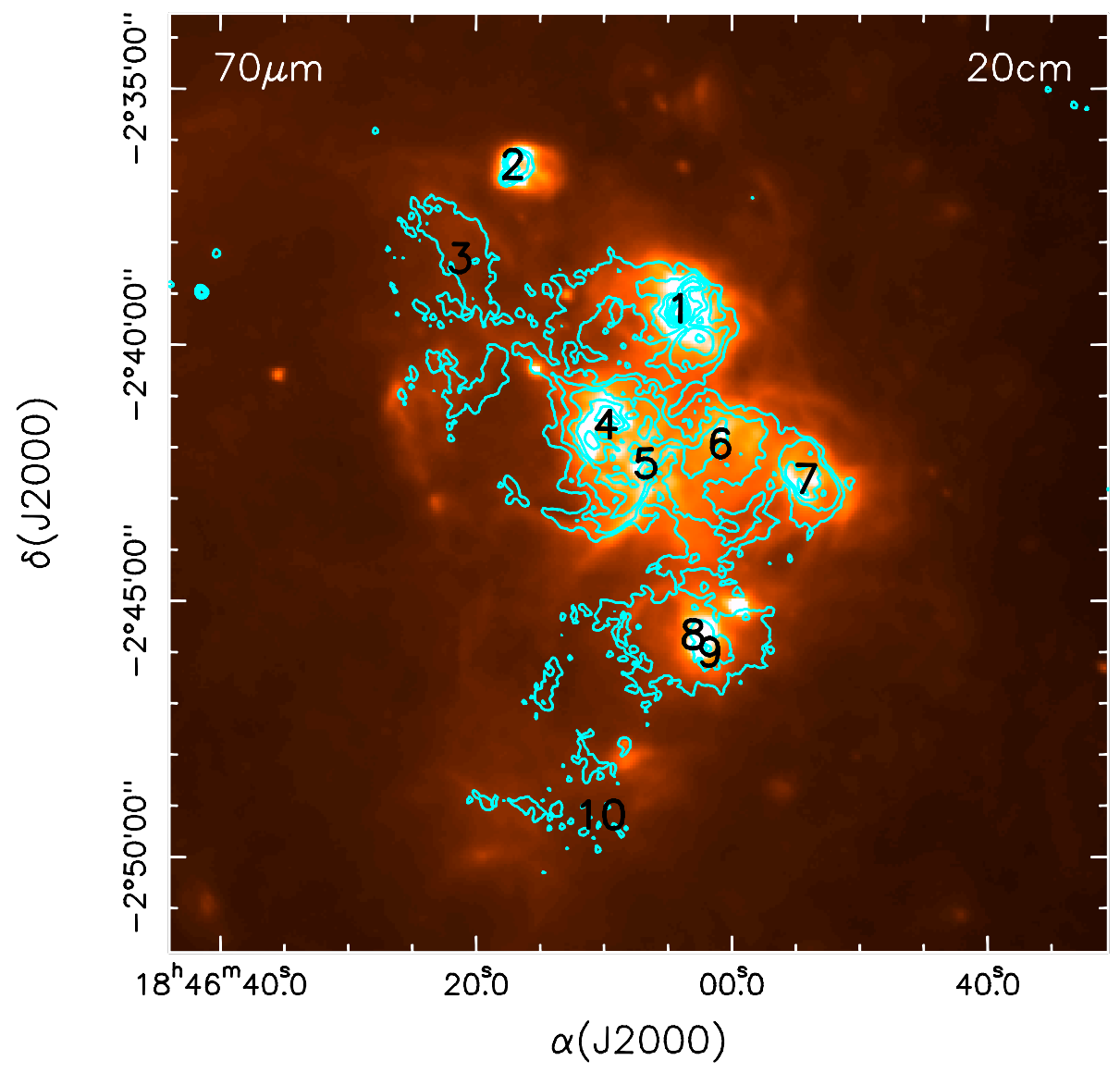

Fig. 6. $20 \mathrm{~cm}$ continuum emission from the MAGPIS survey (cyan contours) of the G29SFR cloud overlaid on the Hi-GAL $70 \mu \mathrm{m}$ emission (colors). Contour levels are 3, 6, 12, 24, 48, 96, and 192 times $1 \mathrm{mJy}^{\text {beam }}{ }^{-1}$. The numbers indicate the positions of the 10 NVSS sources. The synthesized beam of the $20 \mathrm{~cm}$ MAGPIS observations is $66^{\prime \prime} 2 \times 5^{\prime \prime} .4$ at PA $=0^{\circ}$. equilibrium with the surrounding clump environment, are undergoing global collapse to feed a central accretion disk. On the other hand, the clump is defined as the gas cloud that fragments to form a star cluster. These authors propose that fragmentation in these clumps could be inhibited by magnetic fields rather than radiative heating and that the initial conditions of local massive star formation in the Galaxy may be better characterized by surface density values of $\sim 0.2 \mathrm{~g} \mathrm{~cm}^{-2}$ rather than $1 \mathrm{~g} \mathrm{~cm}^{-2}$. This would imply smaller accretion rates and longer formation timescales $\left(>10^{5} \mathrm{yr}\right)$ for massive stars than those predicted my McKee \& Tan (2003).

The mean luminosity estimated, $6.2 \times 10^{3} L_{\odot}$, would correspond to a main-sequence star of spectral type B1 following Table 1 of Mottram et al. (2011), and thus, it also indicates that the Hi-GAL sources associated with the G29-SFR cloud are mostly high-mass sources. Note, however, that this value is an order of magnitude smaller than the average value of $6.7 \times 10^{4} L_{\odot}$ obtained by Beltrán et al. (2006) for a sample of massive protostellar candidates. This is not surprising, taking into account that the bolometric luminosities calculated by these authors are to be considered upper limits because estimated from the IRAS flux densities. The IRAS beam is so large $\left(\sim 2^{\prime}\right)$ that when integrating the flux density for a single protostellar candidate, there might be an important contribution not only from other sources that may fall into such a large beam, but also from inter-clump diffuse emission. The latter contribution is subtracted out when doing the source extraction but is included if one simulates what would be seen with a larger beam like that of IRAS.

The luminosity-to-mass ratio, $L_{\mathrm{bol}} / M_{\mathrm{env}}$, is an important parameter for establishing the age of a source. This ratio is expected to increase with time as more gas is incorporated into the star that becomes more luminous. The mean and median $L_{\mathrm{bol}} / M_{\mathrm{env}}$ values for the sources in the G29-SFR cloud are 23 and $5 L_{\odot} / M_{\odot}$, respectively, which are significantly lower than the average and median values of $99 L_{\odot} / M_{\odot}$ obtained by Beltrán et al. (2006). However, as already mentioned, this discrepancy could be due to the fact that the bolometric luminosities of the sources in the Beltrán et al. sample are likely upper limits because they were estimated from the IRAS fluxes.

\subsection{Centimeter emission associated with the G29-SFR cloud}

Figure 6 shows a zoom-in toward the central region of the G29-SFR cloud. In this figure, the $20 \mathrm{~cm}$ emission of the MultiArray Galactic Plane Imaging Survey (MAGPIS: Helfand et al. 2006) is overlaid on the Hi-GAL $70 \mu$ m emission. The angular resolution of both sets of data is similar, which makes the comparison straightforward. The positions of the ten $21-\mathrm{cm}$ sources associated with the cloud from the NRAO/VLA Sky Survey (NVSS: Condon et al. 1998) catalog at $1.4 \mathrm{GHz}$ are also indicated in the figure. NVSS source \#1 is associated with the G29-UC region and with Hi-GAL source \#242. Table 5 gives the coordinates, flux densities at $21 \mathrm{~cm}$, and major and minor axes of the NVSS sources after deconvolving with the restoring beam of $45^{\prime \prime}$ of the NVSS images. The source fluxes have been obtained from the NVSS catalog instead of estimating them directly from the MAGPIS map at $20 \mathrm{~cm}$ because of the better surface brightness sensitivity of NVSS. The deconvolved sizes for five out of ten sources are upper limits, which indicates that either the source is unresolved or that the emission is too large to properly fit it with just one Gaussian (Condon et al. 1998). The latter is the case for NVSS source \#10, which, as seen in Fig. 6, is very extended and with a very low-level emission, and therefore difficult to fit with a Gaussian. Figure 7 shows the $20 \mathrm{~cm}$ continuum emission overlaid on the 70-350 $\mu \mathrm{m}$ color 


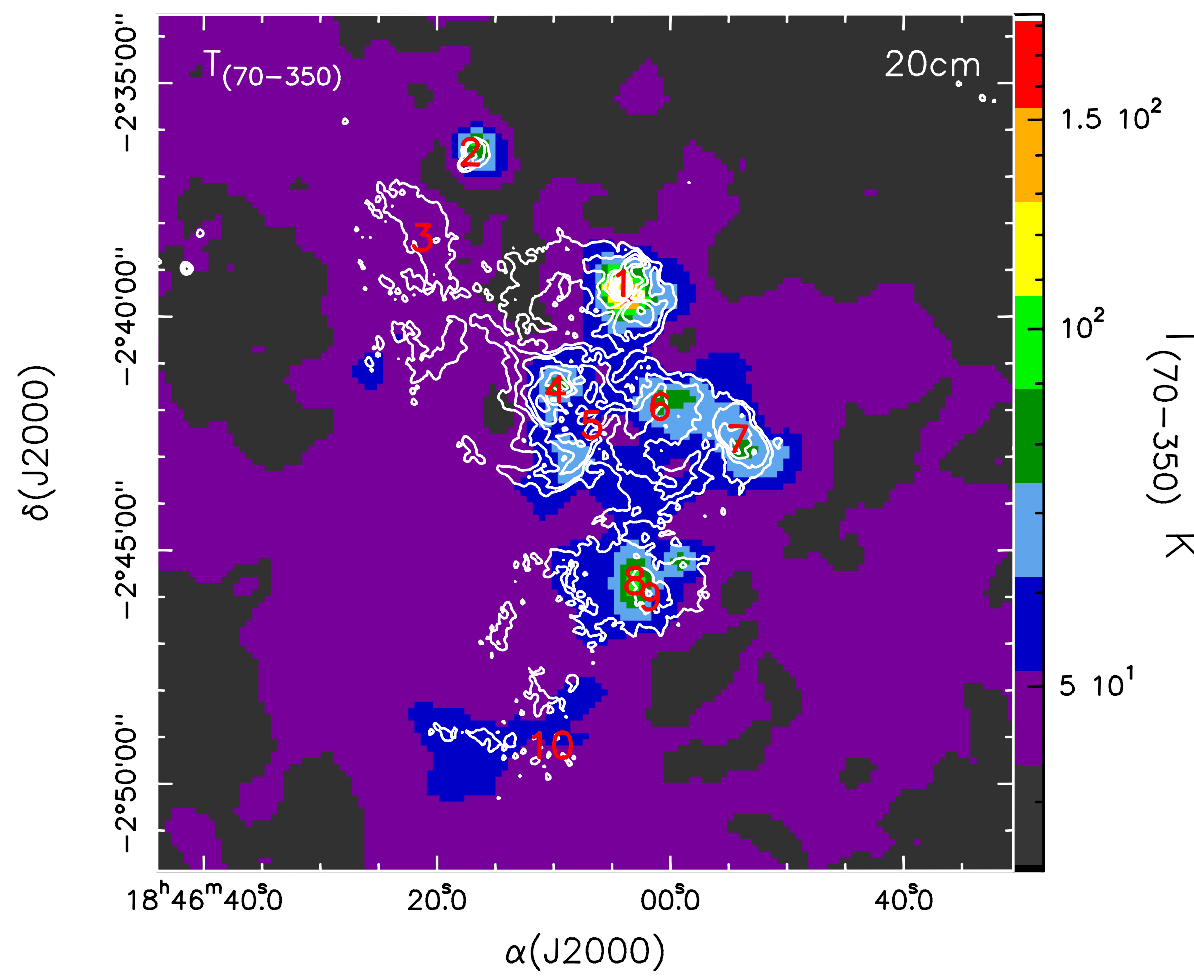

Fig. 7. $20 \mathrm{~cm}$ continuum emission from the MAGPIS survey (blue contours) of the G29-SFR cloud overlaid on the 70-350 $\mu \mathrm{m}$ color temperature map in logarithmic scale (colors). Contour levels and numbers are the same of Fig. 6. temperature, $T_{(70-350)}$, map. To calculate the color-color temperature map, we first smoothed the $70 \mu \mathrm{m}$ map (that with the highest angular resolution: 9.'2) to the resolution of the $350 \mu \mathrm{m}$ map $\left(25^{\prime \prime}\right)$, and then reprojected both maps to the same pixel and map size. These two wavelengths happen to bracket the peak of the SED and are hence most sensitive to temperature changes. Clearly, the color temperature is a proxy for the dust temperature, but may differ significantly from the temperature estimate obtained by fitting the whole SED with a modified blackbody. As seen in Fig. 7, the positions of the NVSS sources, except for the very diffuse NVSS sources \#3 and 10, coincide with local maxima of the color-color temperature.

As seen in Fig. 6, the centimeter emission is well correlated with the $70 \mu \mathrm{m}$ emission, even at the low level emission. Note how both the centimeter and the FIR emission trace the arcs seen eastwards of NVSS sources \#4 and 5. These arcs are shock fronts where hydrogen is ionized, and gives rise to the radio continuum. It is also possible that important shock gas coolants like the [OI $63 \mu \mathrm{m}$ ] line could be in part contaminating the PACS $70 \mu \mathrm{m}$ emission. The fact that the centimeter emission is so extended and well correlated with the dust emission would suggest that it is associated with a group of HII regions that are ionizing and disrupting the cloud. Assuming that the centimeter continuum emission comes from homogeneous optically thin HII regions, we calculated the physical parameters of the 10 NVSS sources (using the formalism of Mezger \& Henderson 1967; and Rubin 1968) and list them in Table 6. Column 1 gives the NVSS number of the source (Table 5), Col. 2 the spatial radius $R$ of the HII region, which was determined from the deconvolved source size (Table 5), Col. 3 the source averaged brightness $T_{\mathrm{B}}$, Col. 4 the electron density $n_{\mathrm{e}}$, Col. 5 the emission measure $E M$, Col. 6 the number of Lyman-continuum photons per second $N_{\text {Ly }}$, Col. 7 the mass of ionized gas $M_{\text {ion }}$, which was calculated assuming a spherical homogeneous distribution, and Col. 8 the spectral type of the ionizing source. The spectral type was computed from the estimated $N_{\text {Ly }}$ and using the tables of Davies et al. (2011) and Mottram et al. (2011), which are for zero age main
Table 5. Position, fluxes, and sizes of the NVSS sources associated with the G29-SFR cloud.

\begin{tabular}{lccccc}
\hline \hline \# Id. & $\begin{array}{c}\alpha(\mathrm{J} 2000) \\
(\mathrm{h} \mathrm{m} \mathrm{s})\end{array}$ & $\begin{array}{c}\delta(\mathrm{J} 2000) \\
\left({ }^{\prime}{ }^{\prime \prime}\right)\end{array}$ & $\begin{array}{c}S_{21 \mathrm{~cm}} \\
(\mathrm{Jy})\end{array}$ & $\begin{array}{c}\text { Major axis } \\
\left({ }^{\prime \prime}\right)\end{array}$ & $\begin{array}{c}\text { Minor axis } \\
\left({ }^{\prime \prime}\right)\end{array}$ \\
\hline $1^{a}$ & 184604.09 & -23919.1 & 2.38 & 26.6 & 20.4 \\
2 & 184617.11 & -23630.0 & 0.025 & $<26.3$ & $<18.4$ \\
3 & 184621.24 & -23820.3 & 0.035 & 63.5 & 31.2 \\
4 & 184609.83 & -24134.9 & 3.06 & 49.3 & 44.7 \\
5 & 184606.69 & -24220.8 & 1.55 & 127.3 & 50.1 \\
6 & 184600.89 & -24157.4 & 0.137 & $<14.2$ & $<14.1$ \\
7 & 184554.17 & -24239.0 & 0.435 & 35.6 & 24.5 \\
8 & 184603.00 & -24541.0 & 0.097 & $<80.3$ & $<21.6$ \\
9 & 184601.67 & -24601.6 & 0.052 & $<15.5$ & $<15.3$ \\
10 & 184610.25 & -24912.3 & 0.010 & $<125.9$ & $<125.0$ \\
\hline
\end{tabular}

Notes. ${ }^{(a)}$ G29-UC and Hi-GAL source \#242.

sequence (ZAMS) stars. Note that if the $21 \mathrm{~cm}$ emission is optically thick, then $T_{\mathrm{B}}, n_{\mathrm{e}}, E M, N_{\mathrm{Ly}}, M_{\mathrm{ion}}$, and therefore, the spectral type should be considered as lower limits. For the sources with upper limits for the deconvolved sizes (Table 5), $R$ and $M_{\text {ion }}$ should be taken as upper limits, while $T_{\mathrm{B}}, n_{\mathrm{e}}$ and $E M$ as lower limits. As seen in Table 6, most of the sources are early B or late $\mathrm{O}$ types. However, the cloud would also contain 3 sources, with one of them being the G29-UC region (NVSS source \#1), with spectral types O5-O6.5. Therefore, it is possible that these massive sources, with their strong winds and radiation pressure, are disrupting and shaping the cloud. This effect may contribute to underestimate the number of ionizing photons and, in turn, the luminosities of the stars. Note that sources \#4 and 5, located at the head of the large arc-like structure seen toward the east, have spectral types $\mathrm{O} 5$ and 06.5 , respectively.

\subsection{Physical parameters as a function of the distance to the NVSS sources in the G29-SFR cloud}

To check whether there is a variation of the Hi-GAL source physical parameters as a function of the distance to the most 
Table 6. Physical parameters of the HII regions in the G29-SFR cloud.

\begin{tabular}{lccccccc}
\hline \hline \# Id. & $\begin{array}{c}R \\
(\mathrm{pc})\end{array}$ & $\begin{array}{c}T_{\mathrm{B}} \\
(\mathrm{K})\end{array}$ & $\begin{array}{c}n_{\mathrm{e}} \\
\left(\mathrm{cm}^{-3}\right)\end{array}$ & $\begin{array}{c}E M \\
\left(10^{5} \mathrm{~cm}^{-6} \mathrm{pc}\right)\end{array}$ & $\begin{array}{c}N_{\mathrm{Ly}} \\
\left(10^{47} \mathrm{~s}^{-1}\right)\end{array}$ & $\begin{array}{c}M_{\text {ion }} \\
\left(M_{\odot}\right)\end{array}$ & $\begin{array}{c}\text { Spectral } \\
\text { Type }\end{array}$ \\
\hline $1^{a}$ & 0.35 & 274 & 2028 & 19 & 84 & 9.0 & O6 \\
2 & $<0.33$ & $>32$ & $>226$ & $>0.23$ & 0.88 & $<0.85$ & $\mathrm{~B} 0$ \\
3 & 0.67 & 11 & 93 & 0.08 & 1.2 & 2.9 & $\mathrm{~B} 0$ \\
4 & 0.71 & 865 & 803 & 6 & 108 & 29 & O5 \\
5 & 1.2 & 152 & 258 & 1 & 55 & 46 & O6.5 \\
6 & $<0.21$ & $>426$ & $>1027$ & $>3$ & 4.8 & $<1.0$ & O9.5 \\
7 & 0.44 & 311 & 607 & 2 & 15 & 5.5 & O8.5 \\
8 & $<0.63$ & $>35$ & $>171$ & $>0.24$ & 3.4 & $<4.3$ & 09.5 \\
9 & $<0.23$ & $>136$ & $>557$ & $>0.96$ & 1.8 & $<0.71$ & B0 \\
10 & $<1.9$ & $>0.4$ & $>10$ & $>0.003$ & 0.35 & $<7.2$ & B0.5 \\
\hline
\end{tabular}

Notes. ${ }^{(a)}$ G29-UC and Hi-GAL source \#242.

massive sources in the G29-SFR cloud, we plotted the distribution of masses, surface densities, luminosities, temperatures, and luminosity-to-mass ratios of the Hi-GAL sources as a function of the distance to the NVSS sources \#1, 4, 5, 6, and 8 (Fig. 8). The NVSS sources \#2, 3, 7, and 10 have not been taken into account because are located close to the border of the cloud. NVSS source \#9 is located very close to NVSS source \#8, and therefore, the distributions should be very similar. The data have been binned in intervals of $\sim 80^{\prime \prime}$. For NVSS source \#1 (G29-UC), only one Hi-GAL source (\#242) is found in the first interval of $\sim 80^{\prime \prime}$, which means that the first point in the plots takes into account only the physical parameters of this source.

The physical parameters of NVSS source \#1 (G29-UC) and its immediate surroundings have the highest values of all the centimeter sources in the G29-SFR cloud. This is evident in Fig. 8 when comparing NVSS source \#1 to sources \#4, 5, 6, and 8, but it is also true for the rest of NVSS sources not shown in this plot. Given the large error bars in Fig. 8, one can only see a marginal trend of the mass and surface density, which seem to decrease with the distance from NVSS source \#1. The surface density is above the minimum value of $0.2 \mathrm{~g} \mathrm{~cm}^{-2}$ needed to form massive stars according to theory (Butler \& Tan 2012), up to a distance of $\sim 150^{\prime \prime}$ from the G29-UC region. A similar marginal trend is seen for NVSS source \#4. Although in this case, the decrease in $M_{\text {env }}$ is even less obvious, and the surface density is slightly above $0.2 \mathrm{~g} \mathrm{~cm}^{-2}$ only in a small region $\left(\lessgtr 80^{\prime \prime}\right)$ surrounding the source. Regarding the luminosity, temperature and luminosityto-mass ratio, again, the highest values are found toward the NVSS source \#1 (G29-UC).

The fact that the most massive and luminous Hi-GAL sources in the cloud are located close to the strongest source in the G29-SFR cloud (\#242 or G29-UC) suggest that there is a privileged area for massive star formation in the cloud. Based on the central location of the G29-UC region inside the G29-SFR cloud (Fig. 2), this indicates that high-mass stars form preferentially at the center of the cloud, as expected. An inhomogeneous density distribution of the cloud, with higher density toward the center of the cloud (maybe already present as an initial condition), could be responsible for this source distribution. This is consistent with the findings of most millimeter continuum surveys.

\section{6. $24 \mu \mathrm{m}$-dark versus $24 \mu \mathrm{m}$-bright sources}

Because star formation does not occur simultaneously all over a cloud, one would expect to find young stellar objects in different evolutionary stages associated with the G29-SFR cloud.
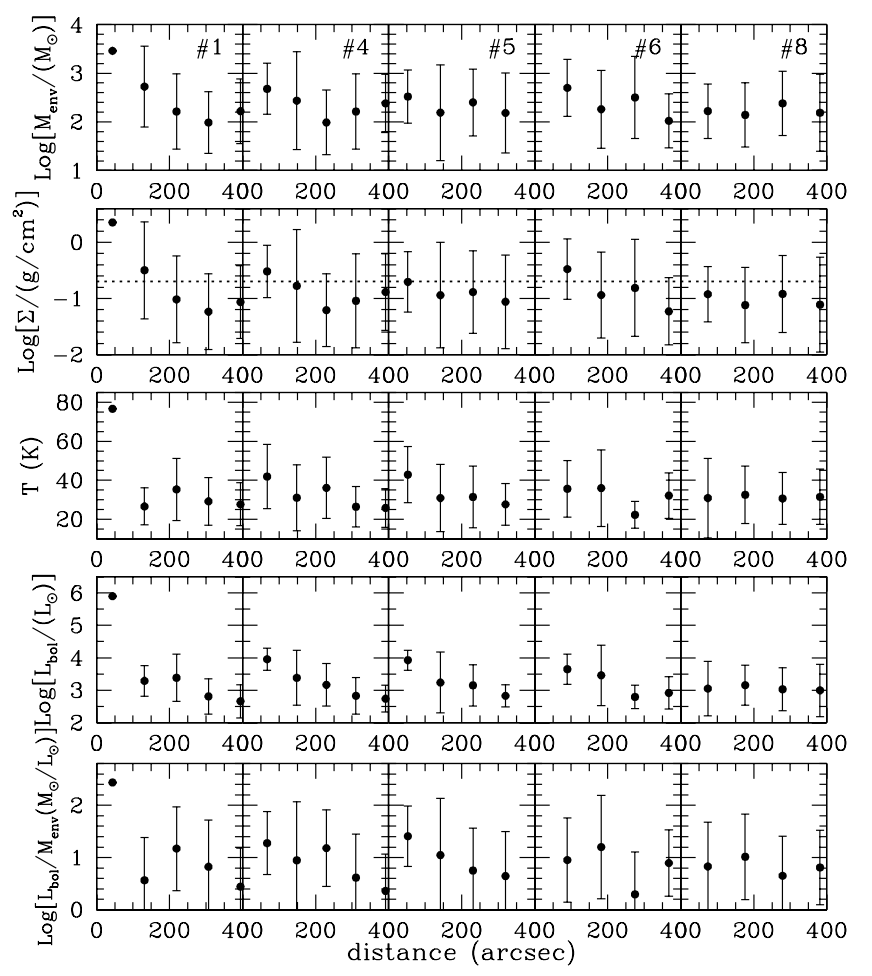

Fig. 8. Distributions of mass, surface density, temperature, luminosity, and luminosity-to-mass ratio as a function of the distance to the NVSS sources in the G29-SFR cloud. The NVSS number (Tables 5 and 6) is indicated in the lefthand upper corner of the upper panels. The dotted line in the surface density distributions indicates the minimum value needed needed to form massive stars according to theory (Butler \& Tan 2012). The first bin contains only one point and, thus, the standard deviation is zero.

To search for differences in the evolutionary stage of the sources, we cross-correlated our Hi-GAL sources with the Spitzer MIPSGAL $24 \mu \mathrm{m}$ catalog. The last column in Table 1 indicates whether a source is associated or not with $24 \mu \mathrm{m}$ emission. Obviously, we counted as associated those sources saturated at $24 \mu \mathrm{m}$, like for example the Hi-GAL source \#242 (G29-UC). Based on this association, we divided the sources into two groups: those without a $24 \mu \mathrm{m}$ counterpart, that we call $24 \mu \mathrm{m}$-dark, and those with a $24 \mu \mathrm{m}$ counterpart, that we call $24 \mu \mathrm{m}$-bright. The former are expected to be the youngest Hi-GAL sources in the cloud. As a result of this cross-correlation we discovered $81 \mathrm{Hi}-\mathrm{GAL}$ sources not associated with $24 \mu \mathrm{m}$ emission and $117 \mathrm{Hi}-\mathrm{GAL}$ sources associated with it. As shown 
M. T. Beltrán et al.: Hi-GAL sources in the G29.96-0.02 cloud

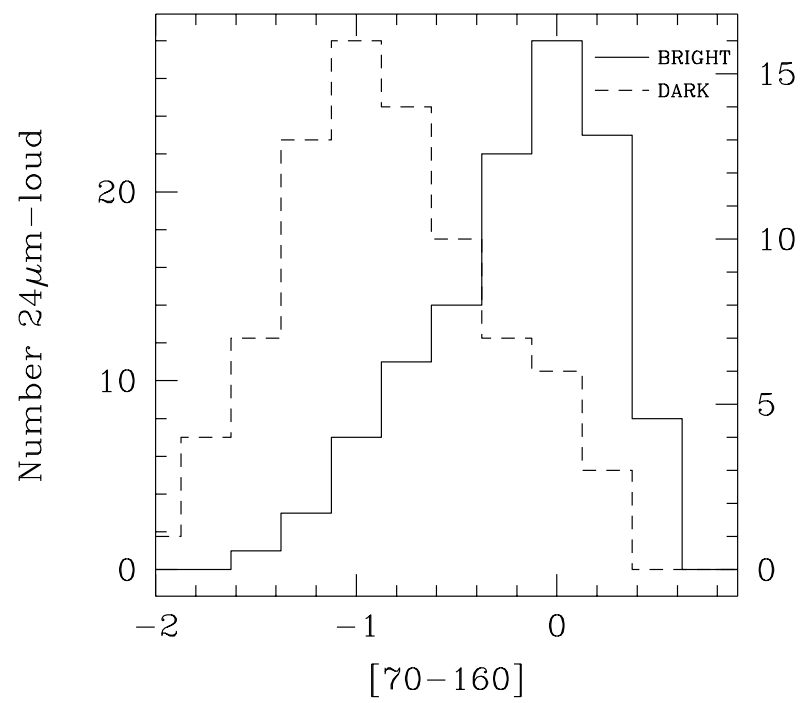

Fig. 9. Histogram of the [70-160] color for $24 \mu \mathrm{m}$-bright (solid line) and $24 \mu \mathrm{m}$-dark (dashed line) sources.

in Fig. 2, both kind of young stellar objects (YSOs) are uniformly distributed over the cloud.

All the sources in our sample have been selected to be detected in all 5 photometric Hi-GAL bands. Therefore, by definition, all the sources have been detected at $70 \mu \mathrm{m}$, which would suggest that most of them, if not all, are protostellar. However, this does not mean that there are no prestellar sources in the G29-SFR cloud (see Pillai et al. 2011). The analysis of the sources not detected at $70 \mu \mathrm{m}$, and likely prestellar, although being highly interesting, goes beyond the scope of the present study. To check whether $24 \mu \mathrm{m}$-dark and $24 \mu \mathrm{m}$-bright sources show any difference in their $70 \mu \mathrm{m}$ fluxes, we plotted and histogram of the [70-160] color for both kind of sources (Fig. 9). As seen in this figure, the [70-160] color of $24 \mu \mathrm{m}$-dark sources is clearly smaller than those of the $24 \mu \mathrm{m}$-bright ones. This indicates that the possible different evolutionary phase of the sources is also supported by the Hi-GAL data.

Figure 10 shows the distribution of radii, masses, surface densities, temperatures, luminosities and luminosity-to-mass ratios for $24 \mu \mathrm{m}$-dark and $24 \mu \mathrm{m}$-bright sources. Table 4 shows the mean and median values for the same physical quantities. One sees that the distributions of the two types of objects are different. A closer inspection of the data using the KolmogorovSmirnov (KS) statistical test shows that, except for the radius distributions, the probability of the mass, surface density, temperature, luminosity, and luminosity-to-mass ratio distributions being the same for $24 \mu \mathrm{m}$-dark and $24 \mu \mathrm{m}$-bright sources is very low ( $P \lessgtr 0.004)$. Therefore, the physical properties of the two groups are statistically different. The temperature, luminosity, and, in particular, the luminosity-to-mass ratio are smaller for the $24 \mu \mathrm{m}$-dark than for the $24 \mu \mathrm{m}$-bright objects, while the mass and the surface density are higher. That $T_{\mathrm{d}}, L_{\mathrm{bol}}$, and $L_{\mathrm{bol}} / M_{\mathrm{env}}$ are smaller for $24 \mu \mathrm{m}$-dark than for $24 \mu \mathrm{m}$-bright sources is consistent with the former being in an earlier evolutionary phase. Figure 10 also shows that a relatively large number of $24 \mu \mathrm{m}$-dark and $24 \mu \mathrm{m}$-bright sources have surface densities high enough to form massive stars according to theory (Butler \& Tan 2012).

The most significant difference between the two groups is found in the value of $L_{\mathrm{bol}} / M_{\mathrm{env}}$. In fact, the mean and median value of $L_{\text {bol }} / M_{\text {env }}$ is $\sim 6$ and $\sim 5$ times lower for the $24 \mu \mathrm{m}$-dark sources compared to the $24 \mu \mathrm{m}$-bright ones, which supports our
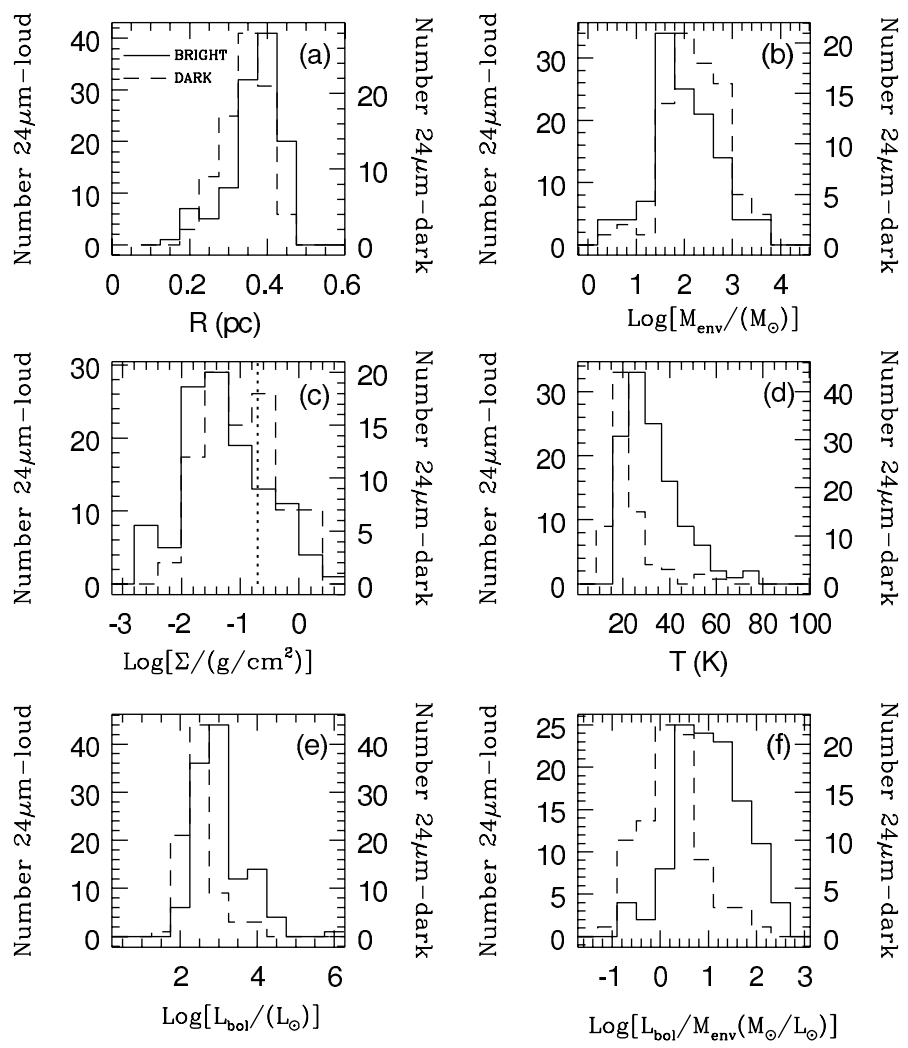

Fig. 10. Histograms of a) radius of the sources; b) mass; c) $\mathrm{H}_{2}$ surface density; d) temperature; e) luminosity; and f) luminosity-to-mass ratio, for $24 \mu \mathrm{m}$-bright (solid line) and $24 \mu \mathrm{m}$-dark (dashed line) sources. The dotted line in panel c) indicates the minimum surface density needed to form massive stars according to theory (Butler \& Tan 2012).

assumption that the sources not associated with $24 \mu \mathrm{m}$ emission are in an earlier evolutionary phase.

We also investigated whether the radii, masses, surface densities, temperatures, luminosities and luminosity-to-mass ratios of the two types of sources show any correlation as a function of the distance to the G29-UC region. Figure 11 indicates that both groups show the same trends, that is, the mass, surface density, and luminosity of the sources marginally decrease when moving away from the G29-UC region, while the size, temperature and luminosity-to-mass ratio, except for the high values close to the G29-UC region, do no significantly change.

\section{Discussion}

\subsection{Evolutionary phase of the sources}

To investigate the stability of the sources, we calculated their Jeans masses, $M_{\mathrm{J}}$, and virial masses, $M_{\text {virial }}$. $M_{\mathrm{J}}$ was calculated following the expression $M_{\mathrm{J}}=[T / 10 \mathrm{~K}]^{3 / 2} \times\left[n_{\mathrm{H}_{2}} / 1 \times\right.$ $\left.10^{4} \mathrm{~cm}^{-3}\right]^{-1 / 2}$, where the dust temperature $T$ was obtained from the SED fitting and the $\mathrm{H}_{2}$ volume density $n_{\mathrm{H}_{2}}$ was calculated assuming that the sources have spherical symmetry (the size of the sources is that obtained from the source extraction process). $M_{\text {virial }}$ was estimated from the line width, $\Delta V$, of ${ }^{13} \mathrm{CO}(1-0)$ toward the position of each source following the expression of MacLaren et al. (1988), $M_{\text {virial }}=0.509 \times d \times \theta \times \Delta V^{2}$, where $d$ is the distance in kpc, $\theta$ is the size of the source in arcsec obtained from the source extraction process, and $\Delta V$ is in $\mathrm{km} \mathrm{s}^{-1}$. The choice of ${ }^{13} \mathrm{CO}(1-0)$ to estimate the virial masses, which could be partially optically thick and therefore overestimate the line 

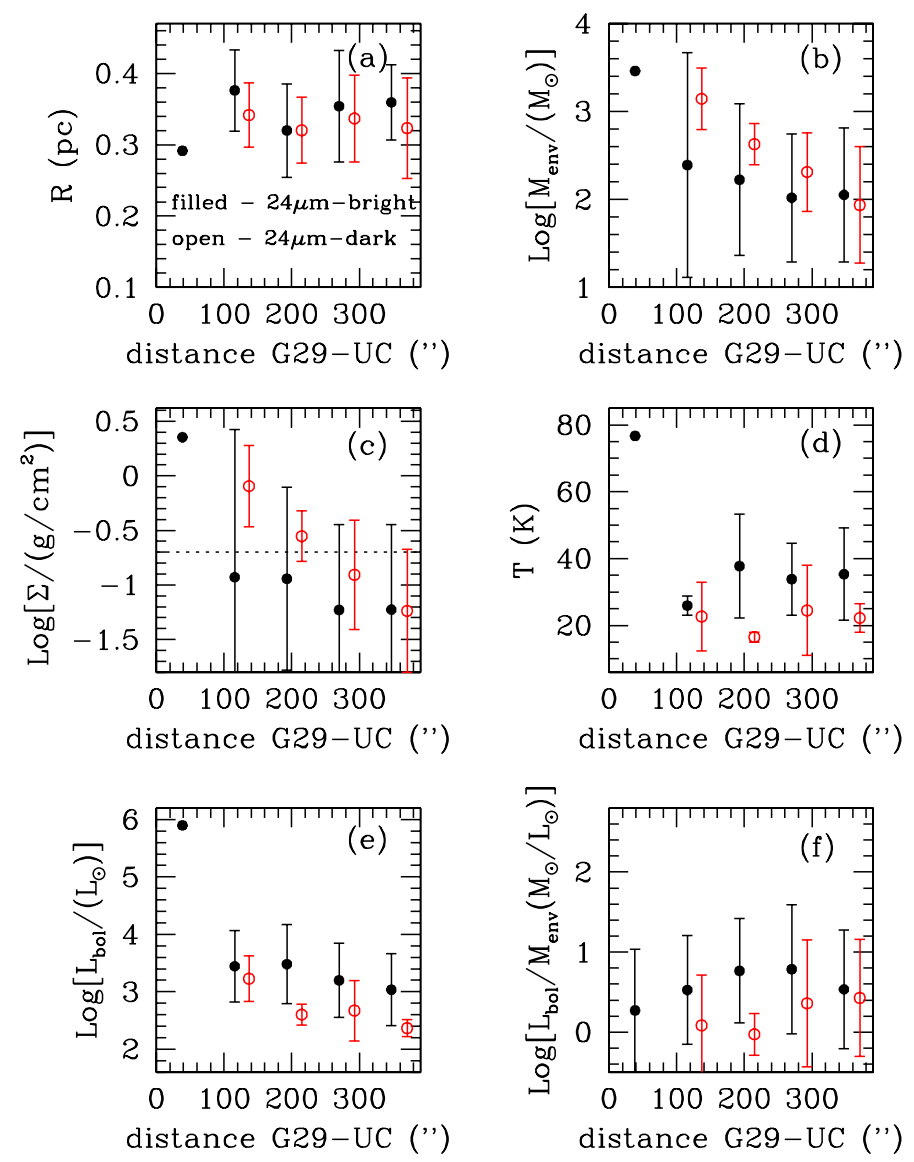

Fig. 11. Distributions of a) radius of the sources; b) mass; c) $\mathrm{H}_{2}$ surface density; d) temperature; e) luminosity; and f) luminosity-to-mass ratio as a function of the distance to the G29-UC region for $24 \mu \mathrm{m}$-bright (filled circles) and $24 \mu \mathrm{m}$-dark (open red circles) sources. The dotted line in the surface density distributions indicates the minimum value needed to form massive stars according to theory (Butler \& Tan 2012). The first bin contains only one point and, thus, the standard deviation is zero.

width, is based on the fact that it is the only molecular tracer covering the whole cloud. To have an idea of how large the overestimate of the line widths could be, we checked the value toward source \#242 (associated with the G29-UC region and G29-HMC core), which has been extensively observed in different molecular tracers. The line width estimated with ${ }^{13} \mathrm{CO}$ is $6.2 \mathrm{~km} \mathrm{~s}^{-1}$ and is similar to the values of $\sim 5.5 \mathrm{~km} \mathrm{~s}^{-1}$ estimated in CS (5-4) and (7-6), and $\mathrm{HCO}^{+}$(3-2) with the JCMT and the IRAM 30-m telescopes (Olmi et al. 1999; Churchwell et al. 2010). $M_{\text {virial }}$ depends on the density profile, and for a power-law density distribution of the type $\rho \propto r^{-p}$, the virial mass should be multiplied by a factor $3(5-2 p) / 5(3-p)$, which is $\leq 1$ for $p<3$. Thus, the values estimated should be taken as upper limits.

Figure 12 shows the $M_{\mathrm{env}}-M_{\mathrm{J}}$ ratio and the $M_{\mathrm{env}}-M_{\text {virial }}$ ratio for all the sources, $24 \mu \mathrm{m}$-bright and $24 \mu \mathrm{m}$-dark. As seen in this plot, almost all the sources have masses well above $M_{\mathrm{J}}$. In particular, $90 \%$ of the $24 \mu \mathrm{m}$-bright sources and $96 \%$ of the $24 \mu \mathrm{m}$ dark ones have masses well above $M_{\mathrm{J}}$. In fact, the mean and median values of the $M_{\mathrm{env}}-M_{\mathrm{J}}$ ratio are 296 and 14 for $24 \mu \mathrm{m}$-bright sources, and 735 and 86 for $24 \mu \mathrm{m}$-dark sources. This indicates that most of the sources in the G29-SFR cloud would be gravitationally supercritical if only supported by thermal pressure, in which case, they should be collapsing. The $M_{\text {env }}-M_{\text {virial }}$ ratio confirms that an additional supporting agent, such as turbulence, is likely acting against gravity in these sources, because only $5 \%$
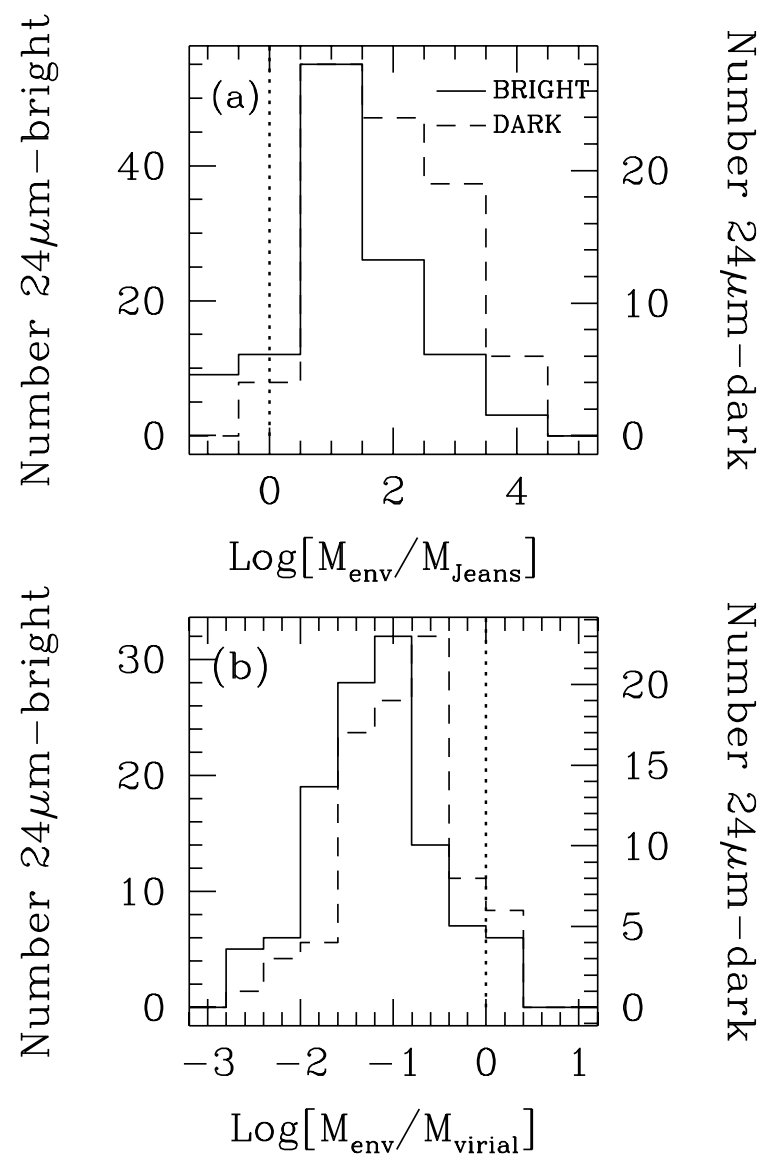

Fig. 12. Histogram of a) the mass-to-Jeans mass ratio and b) mass-tovirial mass ratio for $24 \mu \mathrm{m}$-bright (solid line) and $24 \mu \mathrm{m}$-dark (dashed line) sources. The dotted vertical line indicates a) $M_{\mathrm{env}}=M_{\mathrm{J}}$ and b) $M_{\text {env }}=M_{\text {virial }}$.

(6 out of 117 sources) of the $24 \mu \mathrm{m}$-bright sources and $7 \%$ (6 out of 81 sources) of the $24 \mu \mathrm{m}$-dark ones have masses above the virial mass. The mean and median values of the $M_{\text {env }}-M_{\text {virial }}$ ratio are 0.2 and 0.07 for $24 \mu \mathrm{m}$-bright sources, and 0.3 and 0.1 for $24 \mu \mathrm{m}$-dark sources.

This result seems to be in contrast with the results of other studies of high-mass star-forming clumps, where the mass of the clumps is found to be larger than the virial mass (e.g. Hofner et al. 2000; Fontani et al. 2002). López-Sepulcre et al. (2010) findings for a sample of 29 IR-bright and 19 IR-dark high-mass cluster-forming clumps are similar to ours, although on average their objects are closer to virial equilibrium. What are the sources of uncertainty in our estimate of the $M_{\mathrm{env}}-M_{\text {virial }}$ ratio? The major problem is that very likely the ${ }^{13} \mathrm{CO}$ emission is not tracing the same volume of gas as the $1.2 \mathrm{~mm}$ continuum emission. This means that the ${ }^{13} \mathrm{CO}$ line width may not be representative of the gas contributing to $M_{\mathrm{env}}$. However, to allow for a mean value of $M_{\text {env }} / M_{\text {virial }}=1$, one should shift the distributions in Fig. $12 \mathrm{~b}$ by an order of magnitude, which implies a decrease of the line width by a factor $\sim 3$. This seems too much, as observations of different tracers with different resolutions in high-mass star forming regions reveal changes by only a few $\mathrm{km} \mathrm{s}^{-1}$, for line widths of several $\mathrm{km} \mathrm{s}^{-1}$. Another source of error could be the temperature estimate, which enters almost linearly into the calculation of $M_{\text {env }}$. It is thus difficult to believe that this effect may contribute by more than 20-30\%, by far less than the factor 10 required to match $M_{\text {env }}$ to $M_{\text {virial }}$. Finally, density gradients may affect the estimate of $M_{\text {virial }}$. Assuming 
a power-law density profile as steep as $\rho \propto R^{-2}$, with $R$ radius of the clump, our values of $M_{\text {virial }}$ should decrease only by a factor 0.6 (see MacLaren et al. 1988), still not sufficient to justify the observed ratio $M_{\text {env }}-M_{\text {virial }}$.

We conclude that none of the previous effects can explain the distributions in Fig. 12b. However, it is possible that all of them contribute to the result. While this is certainly possible for a limited number of sources (especially those with $M_{\text {env }} / M_{\text {virial }} \lesssim 1$ ), it seems likely that $M_{\text {env }} / M_{\text {virial }}$ is indeed $<1$ for the majority of the objects.

Assuming that this is the case, it is interesting to note that of the 36 sources located at $\$ 4^{\prime}$ of the G29-UC region, $14 \%$ (5 sources including source \#242: G29-UC) have $M_{\text {env }}>M_{\text {virial }}$. On the other hand, of the remaining 162 sources, which are located at $>4^{\prime}$, only $4 \%$ ( 7 sources), have $M_{\text {env }}>M_{\text {virial }}$. Despite the poor statistics, this result seems to suggest that the sources that should be undergoing collapse and forming stars are preferentially concentrated toward the dominant source in the G29-UC cloud.

The fact that the sources associated with the G29-SFR cloud appear to be in different evolutionary stages is also suggested by the association or not with Spitzer $24 \mu \mathrm{m}$ emission, as already discussed in Sect. 3.6.

To check the validity of this evolutionary phase difference for the sources in the G29-SFR cloud, we decided to use the evolutionary sequence tool of Molinari et al. (2008). These authors have developed an empirical model to describe the premain sequence evolution of YSOs in the high-mass regime based on an $L_{\mathrm{bol}}-M_{\mathrm{env}}$ diagram, where $L_{\mathrm{bol}}$ is the bolometric luminosity of the sources, and $M_{\text {env }}$ the total envelope mass. Based on the model of collapse in turbulence supported cores of McKee \& Tan (2003), which describes the free-fall accretion of material onto a central source as a time-dependent process, Molinari et al. (2008) have constructed evolutionary tracks in the $L_{\mathrm{bol}}-M_{\mathrm{env}}$ diagram. According to this evolutionary sequence, sources in different phases should occupy different regions of the $L_{\mathrm{bol}}-M_{\mathrm{env}}$ diagram. For the high-mass regime, the bolometric luminosity of a YSO evolving toward the ZAMS increases by several orders of magnitude during the accretion phase. Therefore, one would expect $24 \mu \mathrm{m}$-dark sources to have a lower $L_{\text {bol }}$ than the $24 \mu \mathrm{m}$-bright ones for similar $M_{\text {env }}$. Elia et al. (2010) prefer to use the $L_{\text {bol }} / M_{\text {env }}$ ratio versus $M_{\text {env }}$ as a diagnostic, based on the fact that an earlier evolutionary stage source should have smaller $L_{\mathrm{bol}} / M_{\mathrm{env}}$ ratio than more evolved ones.

As seen in Fig. 13, $24 \mu \mathrm{m}$-dark and $24 \mu \mathrm{m}$-bright sources occupy different regions of the $L_{\mathrm{bol}}-M_{\mathrm{env}}$ and $L_{\mathrm{bol}} / M_{\mathrm{env}}-M_{\mathrm{env}}$ diagrams, with $24 \mu \mathrm{m}$-dark sources having lower $L_{\mathrm{bol}}$ and $L_{\mathrm{bol}} / M_{\mathrm{env}}$ for similar $M_{\mathrm{env}}$, as expected. This confirms that the sources not associated with $24 \mu \mathrm{m}$ emission are indeed in an earlier evolutionary phase than those associated. In fact, almost all the $24 \mu \mathrm{m}-$ dark sources occupy a lower part of the accretion phase of the Molinari et al. evolutionary tracks, while the $24 \mu \mathrm{m}$-bright ones are located closer to the ZAMS, as indicated by the end of the ascending tracks.

\subsection{Embedded population in the G29-SFR cloud}

As discussed in the previous section, most of the sources in the G29-SFR cloud seem to be in the main accretion pre-main sequence phase or early ZAMS phase (Fig. 13). This seems to indicate that the population in the G29-SFR cloud, mostly massive sources, should be highly embedded. In a recent work, Faimali et al. (2012) analyze Hi-GAL data on another massive star-forming region G305 and propose a far-IR color criterion to
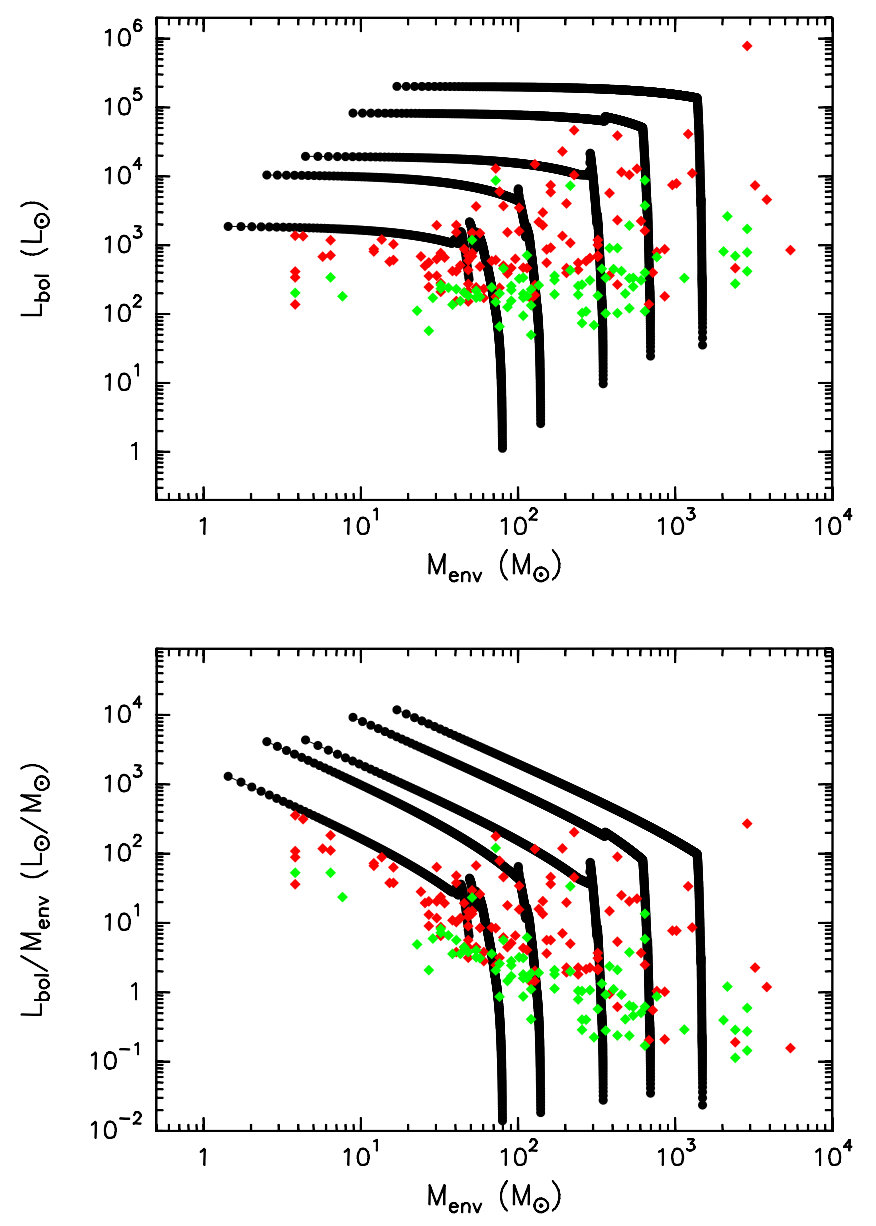

Fig. 13. $L_{\mathrm{bol}}-M_{\mathrm{env}}$ (upper panel) and $L_{\mathrm{bol}} / M_{\mathrm{env}}-M_{\mathrm{env}}$ (lower panel) plots for $24 \mu \mathrm{m}$-bright (red diamonds) and $24 \mu \mathrm{m}$-dark (green diamonds) sources in the G29-SFR cloud. Black lines represent the evolutionary tracks of Molinari et al. (2008) (see Sect. 4.1). The different models are for different initial masses of 80, 140, 350, 700 and $1500 M_{\odot}$ (from left to right).

select massive embedded sources. According to these authors, the [70-500] and the [160-350] colors should be most sensitive to the embedded population. Based on the fact that the embedded massive protostars in G305, associated with typical signposts of massive star formation such as free-free emission, water and/or methanol masers, and $24 \mu \mathrm{m}$ emission, are confined to an area

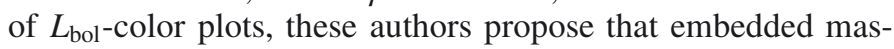
sive star-forming sources, both prestellar and protostellar, should have $[70-500] \geq 1$ and $[160-350] \geq 1.6$ for $L_{\text {bol }}>10^{3} L_{\odot}$. To check whether these selection criteria for embedded massive sources are valid for our sources, we plot the luminosity versus color in Fig. 14. The distribution of sources is very similar to that found by Faimali et al. (2012) for the sources in G305. In the G29-SFR cloud, we found $4624 \mu \mathrm{m}$-bright and $724 \mu \mathrm{m}$-dark sources that satisfy the criterion for embedded massive star candidates, a number similar to that found by Faimali et al. (2012) in G305. This would indicate that only $\sim 27 \%$ of the population in the G29-SFR cloud would be embedded massive star candidates. However, as previously mentioned, most of the sources in the G29-SFR cloud seem to be pre-main sequence sources in the main accretion phase or early ZAMS phase, and therefore, embedded. One possible explanation for this discrepancy could be that most of these sources in the G29-SFR cloud have $L_{\text {bol }}<10^{3} L_{\odot}$, and therefore lie by definition outside the selection criterion area. However, by doing this, the selection criterion 

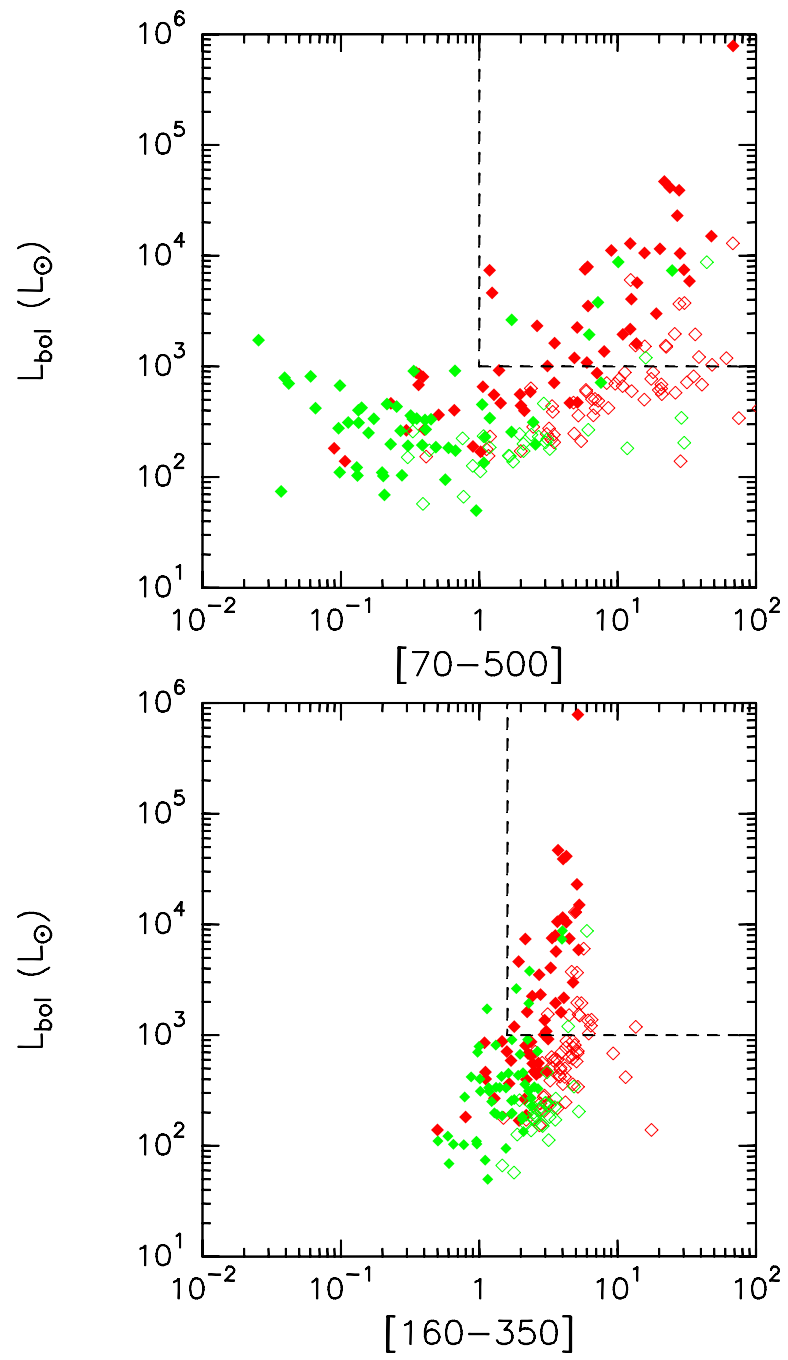

Fig. 14. Luminosity-color plots for the Hi-GAL $24 \mu \mathrm{m}$-bright (red diamonds) and $24 \mu \mathrm{m}$-dark (green diamonds) sources in the G29-SFR cloud. The empty red and green diamonds indicate sources with $M_{\text {env }}<$ $100 M_{\odot}$. Dashed lines indicate the threshold of the area defined by Faimali et al. (2012) for selecting embedded massive YSOs, at a luminosity $>10^{3} L_{\odot}$.

would miss those young massive embedded protostars in a very early evolutionary phase that have not yet reached their final luminosity (see Fig. 13).

The second problem with the Faimali et al. (2012) selection criterion is that, as shown in Fig. 14, there are a few sources that are clearly not massive $\left(M_{\text {env }}<100 M_{\odot}\right)$ and have $L_{\text {bol }}>10^{3} L_{\odot}$ (see Fig. 14) that would fall inside the massive embedded population area. If we lower the limit to $M_{\text {env }}<50 M_{\odot}$, there are still 8 $24 \mu \mathrm{m}$-bright sources that would satisfy the criterion. Therefore, all this suggests that the far-IR color selection criterion for embedded massive YSOs of Faimali et al. (2012) cannot be applied in all the massive star forming regions.

\subsection{The star formation efficiency and rate}

Observations of OB associations and Giant Molecular Clouds indicate that the overall star formation efficiency, $\mathrm{SFE}=$ $M_{\text {stars }} /\left(M_{\text {stars }}+M_{\text {cloud }}\right)$, is very low, $3-4 \%$ (Evans \& Lada 1991 ; Lada 1999). To estimate the SFE in the G29-SFR cloud, we first need the total gass mass of the cloud, $M_{\text {cloud }}$, and the mass of the stars, $M_{\text {stars }}$. The former can be estimated from the Hi-GAL data, while $M_{\text {stars }}$ can be calculated by assuming that the emission in the G29-SFR cloud is consistent with that of a stellar cluster. To check this, we calculated the Lyman continuum, $N_{\text {Ly }}$, of the cloud by measuring the radio flux at $20 \mathrm{~cm}$, and compared this value with the bolometric luminosity, $L_{\mathrm{bol}}$, of the cloud. $L_{\mathrm{bol}}$ was calculated integrating the Hi-GAL emission, inside the same area used to estimate the centimeter flux, in the $5 \mathrm{Hi}-\mathrm{GAL}$ bands and fitting the SED with a modified blackbody. The total radio flux at centimeter wavelengths is $30.6 \mathrm{Jy}$, which corresponds to $N_{\text {Ly }}=1.08 \times 10^{50} \mathrm{~s}^{-1}$. The total $L_{\text {bol }}$ is $2.2 \times 10^{6} L_{\odot}$. For comparison, the sum of $L_{\mathrm{bol}}$ of all the sources that fall inside the area used to estimate the radio flux at $20 \mathrm{~cm}$ is $1.1 \times 10^{6} L_{\odot}$. These values are consistent with the expected $N_{\mathrm{Ly}}$ and $L_{\mathrm{bol}}$ of a stellar cluster according to the simulations of a large collection $\left(10^{6}\right)$ of clusters with sizes ranging from 5 to 500000 stars each (Testi, priv. comm.; see Sánchez-Monge et al. 2013 for a description of the cluster generation). For each cluster simulated, the total mass, bolometric luminosity, maximum stellar mass and integrated Lyman continuum are computed. For a bolometric luminosity of $2.2 \times 10^{6} L_{\odot}, 90 \%$ of the simulated clusters have a total stellar mass $M_{\text {stars }}$ between 600 and $4170 M_{\odot}$. The total gas mass of the cloud, estimated by fitting a modified blackbody to the integrated emission of the cloud, inside the same area used to estimate the radio flux at $20 \mathrm{~cm}$, at the Herschel wavelengths, is $8 \times 10^{4} M_{\odot}$. Therefore, the overall SFE of the G29-SFR cloud ranges from 0.7 to $5 \%$, as low as that estimated in other molecular clouds (Evans \& Lada 1991). For comparison, the sum of the masses of all the sources that fall inside the area used to estimate the centimeter flux is slightly smaller $3 \times 10^{4} M_{\odot}$, and the SFE slightly higher, from 2 to $12 \%$.

The star formation rate of the cloud can be estimated as $S F R=\left(M_{\text {cloud }} \times \mathrm{SFE}\right) / t$, where $t$ is the star formation timescale needed for the protostars to reach the ZAMS. To compare our study of the G29-SFR cloud with that of Faimali et al. (2012), we assume the same timescale of $0.5 \mathrm{Myr}$ used by these authors, which is based on a steady-state star formation model (Offner \& McKee 2011). The SFR obtained for the G29-SFR cloud ranges from 0.001 to $0.008 M_{\odot} \mathrm{yr}^{-1}$. These values are smaller than those of $0.01-0.02 M_{\odot} \mathrm{yr}^{-1}$ estimated by Faimali et al. (2012) for the G305 cloud, but consistent with the values of $\sim 0.0002-0.001 M_{\odot} \mathrm{yr}^{-1}$ estimated by Veneziani et al. (2013) for the whole $l=30^{\circ}$ SDP field, and with the SFRs of $\sim 0.0005$ to $\sim 0.008 M_{\odot} \mathrm{yr}^{-1}$ estimated for Galactic HII regions by Chomiuk \& Povich (2011). The fact that the SFR of the Milky Way is of about $2 M_{\odot} \mathrm{yr}^{-1}$ (Chomiuk \& Povich 2011), indicates that hundreds to a few thousands of molecular clouds similar to the G29-SFR cloud are needed to account for the Galactic star formation rate.

\subsection{The clump mass function}

Figure 15 shows the mass spectrum of the sources in the G29-SFR cloud. Olmi et al. (2013) have analyzed the whole $l=30^{\circ} \mathrm{SDP}$ field and estimated a statistical mass completeness limit, from the $160 \mu \mathrm{m}$ maps at the $80 \%$ confidence level, of $73 M_{\odot}$ for a temperature of $20 \mathrm{~K}$, a dust mass absorption coefficient $\kappa_{0}=11 \mathrm{~cm}^{2} \mathrm{~g}^{-1}$, evaluated at $\nu_{0}=c / 250 \mu \mathrm{m}$, and a gas-to-dust ratio of 100 (Martin et al. 2012), a dust emissivity index of 2, and a median distance for the whole field of $7.6 \mathrm{kpc}$. Assuming a distance of $6.2 \mathrm{Kpc}$ for the G29-SFR cloud, the mass completeness limit is of $\sim 49 M_{\odot}$.

If the source mass distribution can be represented by a power law of the type $\mathrm{d} N / \mathrm{d} M \propto M_{\text {env }}^{-\alpha}$, then the histogram of the mass spectrum can be fitted with a straight line of slope $-\alpha$. The solid line in the figures corresponds to $\alpha=2.35$, i.e., the 

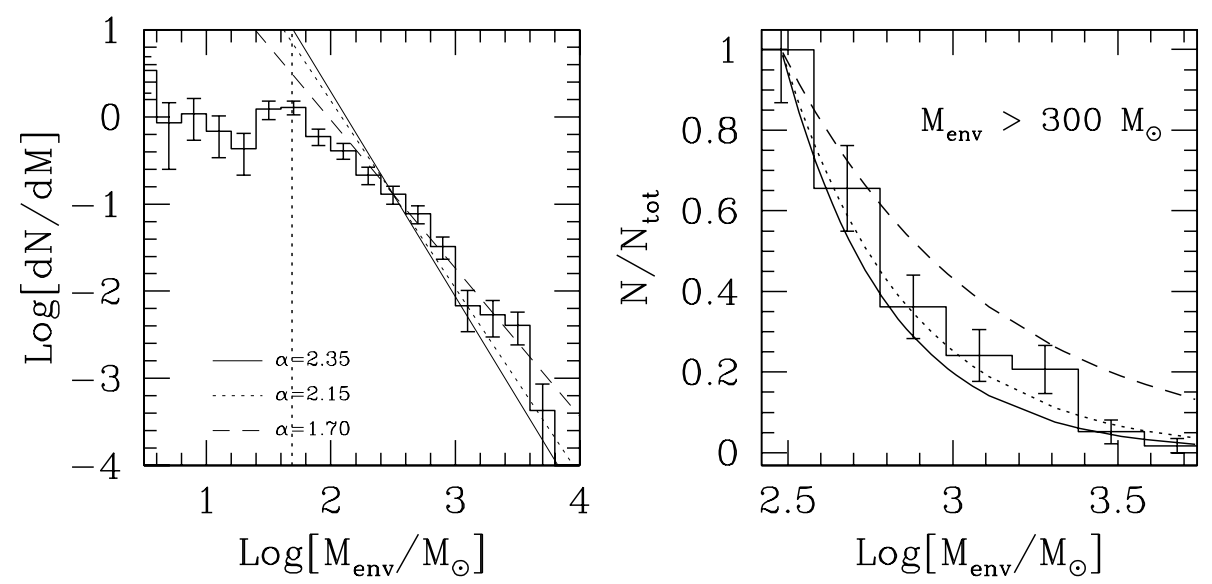

Fig. 15. Left panel: the mass spectrum of the sources in the G29-SFR cloud. The solid line represents the Salpeter IMF, $\mathrm{d} N / \mathrm{d} M \propto M_{\mathrm{env}}^{-2.35}$, the dotted line is a -2.15 power law, and the dashed line is a -1.7 power law. The vertical dotted line indicates the completeness limit of $49 M_{\odot}$ for a temperature of $20 \mathrm{~K}$. Right panel: the normalized cumulative mass distribution of sources with masses above $300 M_{\odot}$ (well above the completeness limit: see Sect. 4.4). The solid, dotted, and dashed lines are the same as in the left panel.

Salpeter (1955) Initial Mass Function (IMF), and the dashed line to $\alpha=1.70$, corresponding to the mass function of molecular clouds derived from gas, mainly $\mathrm{CO}$, observations (e.g. Kramer et al. 1998). The dotted line corresponds to the best-fit powerlaw index of $\alpha=2.15 \pm 0.30$ obtained with a procedure that implements both the discrete and continuous maximum likelihood estimator for fitting the power-law distribution to data, along with a goodness-of-fit based approach to estimating the lower cutoff of the data (see Clauset et al. 2009; and Olmi et al. 2013 , for a detailed description of this method). This lower cutoff will be indicated here as $M_{\text {inf }}$, which will thus represent the value below which the behavior of the distribution departs from a power-law. Following Clauset et al. (2009), we have chosen the value of $M_{\text {inf }}$ that makes the probability distributions of the measured data and the best-fit power-law model as similar as possible above $M_{\text {inf }}$. In order to quantify the difference between these probability distributions, the Kolmogorov-Smirnov statistics is used. The value of $M_{\mathrm{inf}}$ for the sources in the G29-SFR cloud is $\sim 300 \pm 130 M_{\odot}$. This is well above the mass completeness limit. The right panel shows the normalized cumulative mass distribution of the 58 sources with masses above $M_{\text {inf }}$.

The best-fit power-law index $\alpha$ of 2.15 obtained for the G29-SFR cloud is the same obtained by Olmi et al. (2013) for the whole $l=30^{\circ}$ SDP field. $M_{\text {inf }}$ is consistent within the errors with the value of $200 \pm 79 M_{\odot}$ obtained for the whole field. The power-law index is also consistent with the value of 2.20 obtained by the same authors for $l=59^{\circ}$, the second SDP field. $M_{\text {inf }}$ for this field, $7.3 \pm 2.2 M_{\odot}$, is much lower than the value of $\sim 300 M_{\odot}$ estimated for the G29-SFR cloud, but this is not surprising taking into account that the $l=59^{\circ}$ region contains mostly low- to intermediate-mass sources (the median mass for this field is of about $2.1 M_{\odot}$ : Olmi et al. 2013). These values of the power-law index $\alpha$ agree with the typical values found by Swift \& Beaumont (2010), for CMFs of both low- and highmass star-forming regions. This suggests that from the shape of the CMF it is not possible to foresee a different evolution toward the IMF for high- and low-mass star-forming clumps (Olmi et al. 2013).

The value of $\alpha=2.15 \pm 0.30$ is also consistent within the errors with the value of 2.35 of the stellar IMF (Salpeter 1955). The observational similarity between the CMF and the IMF, first noted by Motte et al. (1998) for the low-mass star-forming region $\rho$ Ophiuchi, has been since then observed in many other low-mass star-forming regions (e.g. Simpson et al. 2008, and references therein). This similar behavior has inspired the idea that gravitational fragmentation plays a key role in determining the final mass of the stars, that is, the IMF, in clustered regions (Motte et al. 1998). That the CMF of high-mass star-forming regions mimics the stellar IMF (this work; Beltrán et al. 2006) seems to suggest that also in this case, the fragmentation of massive clumps may determine the IMF and the masses of the final stars. In other words, the processes that determine the clump mass spectrum might be self-similar across a broad range of clump and parent cloud masses.

\section{Conclusions}

We have conducted a far-infrared (FIR) study of the G29-SFR cloud using the Hi-GAL data at 70, 160, 250, 350, and $500 \mu \mathrm{m}$ aimed at identifying the sources associated with this high-mass star-forming region and estimate their physical properties.

A total of 198 sources have been detected in all 5 Hi-GAL bands. The mean and median values of their physical properties are 0.36 and $0.36 \mathrm{pc}$ for the radius, 379 and $115 M_{\odot}$ for the mass, 0.24 and $0.06 \mathrm{~g} \mathrm{~cm}^{-2}$ for the surface density, 29 and $25 \mathrm{~K}$ for the temperature, $6.2 \times 10^{3}$ and $470 L_{\odot}$ for the luminosity, and 23 and $5 L_{\odot} / M_{\odot}$ for the luminosity-to-mass ratio.

The G29-SFR cloud is associated with 10 NVSS sources and with extended centimeter continuum emission well correlated with the $70 \mu \mathrm{m}$ emission. This suggests that the cloud would contain a group of HII regions that are ionizing and disrupting the cloud. Assuming that the centimeter continuum emission comes from homogeneous optically thin regions, we estimated that most of the NVSS sources would be early B or late O types. The cloud would also contain 3 sources, with one of them being that associated with the G29-UC region, with spectral types O5-O6.5. The study of the distribution of masses, surface densities, luminosities, temperatures, and luminosity-to-mass ratios of the Hi-GAL sources as a function of the distance to the NVSS sources indicates that the most massive and luminous sources in the cloud are located close to the G29-UC region. This could suggest that there is a privileged area for massive star formation toward the center of the G29-SFR cloud.

There are 117 Hi-GAL sources associated with $24 \mu \mathrm{m}$ emission, called $24 \mu \mathrm{m}$-bright, and 87 sources not associated, called $24 \mu \mathrm{m}$-dark. Both groups are uniformly distributed over the cloud. The radius of $24 \mu \mathrm{m}$-dark and $24 \mu \mathrm{m}$-bright sources is similar, the temperature and luminosity are smaller for the $24 \mu \mathrm{m}$-dark than for the $24 \mu \mathrm{m}$-bright objects, and the mass and surface density are higher. The luminosity-to-mass ratio is 25-6 times lower for $24 \mu \mathrm{m}$-dark sources. The $24 \mu \mathrm{m}$-dark and $24 \mu \mathrm{m}$-bright sources occupy different regions of the $L_{\mathrm{bol}}-M_{\mathrm{env}}$ and $L_{\mathrm{bol}} / M_{\mathrm{env}}-M_{\mathrm{env}}$ diagrams, with the $24 \mu \mathrm{m}$-dark sources having lower $L_{\mathrm{bol}}$ and $L_{\mathrm{bol}} / M_{\mathrm{env}}$ for similar $M_{\mathrm{env}}$, as expected. All this suggests that the sources not associated with $24 \mu$ m emission are in an earlier evolutionary phase than those associated. This 
is supported by the fact that the [70-160] color of $24 \mu \mathrm{m}$-dark sources is clearly smaller than that of the $24 \mu \mathrm{m}$-bright ones.

Almost all the Hi-GAL sources in the G29-SFR cloud have masses well above the Jeans mass and would be gravitationally supercritical if only supported by thermal pressure. However, only $\sim 6 \%$ of the sources have masses above the virial mass, which confirms that an additional supporting agent, such as turbulence, might be acting against gravity in these sources. The percentage of sources with masses larger than the virial mass is clearly higher for those located at $\$ 4^{\prime}$ of the G29-UC region. This suggests that the sources that should be undergoing collapse and forming stars are preferentially concentrated toward the dominant source in the cloud.

The overall SFE of the G29-SFR cloud ranges from 0.7 to $5 \%$, and it is as low as that estimated in other molecular clouds. The SFR ranges from 0.001 to $0.008 M_{\odot} \mathrm{yr}^{-1}$ and is consistent with the values estimated for Galactic HII regions. To account for the SFR of $2 M_{\odot} \mathrm{yr}^{-1}$ of the Milky Way, hundreds to a few thousands of molecular clouds similar to the G29-SFR cloud would be needed.

The mass spectrum of the Hi-GAL sources with masses above $300 M_{\odot}$, well above the completeness limit, can be wellfitted with a power law of slope $\alpha=2.15 \pm 0.30$, consistent with the values obtained by Olmi et al. (2013) for the whole $l=30^{\circ}$, associated with high-mass star formation, and $l=59^{\circ}$, associated with low- to intermediate-mass star formation, Hi-GAL SDP fields. The observational similarity of the CMF for lowand high-mass star-forming regions suggests that from the $\mathrm{CMF}$ itself is not possible to predict a different evolution of the clumps toward the IMF. The fact that the CMF of the G29-SFR cloud mimics, within the errors, the stellar IMF suggests a self-similar process which determines the shape of the mass spectrum over a broad range of masses, from stellar to cluster size scales.

Acknowledgements. It is a pleasure to thank Annie Zavagno for critically reading the manuscript. Hi-GAL data processing and analysis has been possible thanks to the Italian Space Agency support via contract I/038/080/0. SPIRE has been developed by a consortium of institutes led by Cardiff Univ. (UK) and including: Univ. Lethbridge (Canada); NAOC (China); CEA, LAM (France); IFSI, Univ. Padua (Italy); IAC (Spain); Stockholm Observatory (Sweden); Imperial College London, RAL, UCL-MSSL, UKATC, Univ. Sussex (UK); and Caltech, JPL, NHSC, Univ. Colorado (USA). This development has been supported by national funding agencies: CSA (Canada); NAOC (China); CEA, CNES, CNRS (France); ASI (Italy); MCINN (Spain); SNSB (Sweden); STFC, UKSA (UK); and NASA (USA). PACS has been developed by a consortium of institutes led by MPE (Germany) and including UVIE (Austria); KU Leuven, CSL, IMEC (Belgium); CEA, LAM (France); MPIA (Germany); INAFIFSI/OAA/OAP/OAT, LENS, SISSA (Italy); IAC (Spain). This development has been supported by the funding agencies BMVIT (Austria), ESAPRODEX (Belgium), CEA/CNES (France), DLR (Germany), ASI/INAF (Italy), and CICYT/MCYT (Spain). This publication makes use of data products from the Wide-field Infrared Survey Explorer, which is a joint project of the University of California, Los Angeles, and the Jet Propulsion Laboratory/California Institute of Technology, funded by the National Aeronautics and Space Administration. This research made use of data products from the Midcourse Space Experiment. Processing of the data was funded by the Ballistic Missile Defense Organization with additional support from NASA Office of Space Science. This research has also made use of the NASA/IPAC Infrared Science Archive, which is operated by the Jet Propulsion Laboratory, California Institute of Technology, under contract with the National Aeronautics and Space Administration.

\section{References}

Bally, J., Anderson, L. D., Battersby, C., et al. 2010, A\&A, 518, L90 Battersby, C., Bally, J., Ginsburg, A., et al. 2011, A\&A, 535, A128
Beltrán, M. T., Brand, J., Cesaroni, C., et al. 2006, A\&A, 447, 221 Beltrán, M. T., Cesaroni, C., Neri, R., \& Codella, C. 2011, A\&A, 525, A151 Beuther, H., Zhang, Q., Bergin, E. A., et al. 2007, A\&A, 468, 1045 Butler, J. B., \& Tan, J. C. 2012, ApJ, 745, 5

Cesaroni, R., Churchwell, E., Hofner, P., et al. 1994, A\&A, 288, 903 Cesaroni, R., Hofner, P., Walmsley, C. M., \& Churchwell, E. 1998, A\&A, 331, 709

Chapin, E. L., Ade, P. A. R., Bock, J. J., et al. 2008, ApJ, 681, 428

Chomiuk, L., \& Povich, M. S. 2011, AJ, 142, 197

Churchwell, E., Sievers, A., \& Thum, C. 2010, A\&A, 513, A9 Clauset, A., Shalizi, C. R., \& Newman, M. E. J. 2009, SIAM Rev., 51, 661

Condon, J. J., Cotton, W. D., Greisen, E. W., et al. 1998, AJ, 115, 1693 Davies, B., Hoare, M., Lumsden, S. L., et al. 2011, MNRAS, 416, 972

De Buizer, J. M., Watson, A. M., Radomski, J. T., Piña, R. K., \& Telesco, C. M. 2002, ApJ, 564, L101

Elia, D., Schisano, E., Molinari, S., et al. 2010, A\&A, 518, L97

Evans, N. J. II, \& Lada, E. A. 1991 in Fragmentation of Molecular Clouds and Star Formation, eds. E. Falgarone, \& G. Duvert (Dordretch: Kluwer Academic Publishers), IAU Symp., 147, 293

Faimali, A., Thompson, M. A., Hindson, L., et al. 2012, MNRAS, 426, 402 Fontani, F., Cesaroni, R., Caselli, P., \& Olmi, L. 2002, A\&A, 389, 603 Giannini, T., Elia, D., Lorenzetti, D., et al. 2012, A\&A, 539, A156

Helfand, D. J., Becker, R. H., White, R. L., Fallon, A., \& Tuttle, S. 2006, AJ, 131,2525

Hofner, P., Wyrowski, F., Walmsley, C. M., \& Churchwell, E. 2000, ApJ, 536, 393

Jackson, J. M., Rathborne, J. M., Shah, R. Y., et al. 2006, ApJS, 163, 145

Kirk, J. M., Polehampton, E., Anderson, L. D., et al. 2010, A\&A, 518, L82

Kramer, C., Stutzki, J., Röhring, R., \& Corneliussen, U. 1998, A\&A, 329, 249

Kramer, C., Richer, J., Mookerjea, B., Alves, J., \& Lada, C. 2003, A\&A, 399, 1073

Krumholz, M. R., \& McKee, C. F. 2008, Nature, 451, 1082

Lada, C. J. 1999, in The Origin of Stars and Planetary Systems, eds. C. J. Lada, \& N. D. Kylafis (Kluwer Academic Publishers), 143

López-Sepulcre, A., Cesaroni, R., \& Walmsley, C. M. 2010, A\&A, 517, A66 MacLaren, I., Richardson, K. M., \& Wolfendale, A. W. 1988, ApJ, 333, 821

Martin, P. G., Roy, A., Bontemps, S., et al. 2012, ApJ, 751, 28

Maxia, C., Testi, L., Cesaroni, R., \& Walmsley, C. M. 2001, A\&A, 371, 287

McKee, C. F., \& Tan, J. C. 2003, ApJ, 585, 850

Mezger, P. G., \& Henderson, A. P. 1967, ApJ, 147, 471

Molinari, S., Brand, J., Cesaroni, R., \& Palla, F. 2000, A\&A, 355, 617

Molinari, S., Pezzuto, S., Cesaroni, R., et al. 2008, A\&A, 481, 345

Molinari, S., Swinyard, B., Bally, J., et al. 2010, A\&A, 518, L100

Molinari, S., Schisano, E., Faustini, F., et al. 2011, A\&A, 530, A133

Motte, F., André, P., \& Neri, R. 1998, A\&A, 336, 150

Mottram, J. C., Hoare, M. G., Davies, B., et al. 2011, ApJ, 730, 33

Netterfield, C. B., Ade, P. A. R., Bock, J. J., et al. 2009, ApJ, 707, 1824

Offner, S. S. R., \& McKee, C. F. 2011, ApJ, 736, 56

Olmi, L., \& Cesaroni, R. 1999, A\&A, 352, 266

Olmi, L., Cesaroni, R., Hofner, P., et al. 2003, A\&A, 407, 225

Olmi, L., Ade, P. A. R., Anglés-Alcázar, D., et al. 2009, ApJ, 707, 1836

Olmi, L., Anglés-Alcázar, D., Elia, D., et al. 2013, A\&A, 551, A111

Pilbratt, G. L., Riedinger, J. R., Passvogel, T., et al. 2010, A\&A, 518, L1

Pillai, T., Kauffmann, J., Wyrowski, F., et al. 2011, A\&A, 530, 118

Pratap, P., Megeath, S. T., \& Bergin, E. A. 1999, ApJ, 517, 799

Price, S. D., Egan, M. P., Carey, S. J., Mizuno, D. R., \& Kuchar, T. A. 2001, AJ, 121,2819

Rubin, R. H. 1968, ApJ, 154, 391

Russeil, D., Pestalozzi, M., Mottram, J. C., et al. 2011, A\&A, 526, A151

Salpeter, E. E. 1955, ApJ, 121, 16

Sánchez-Monge, A., Beltrán, M. T., Cesaroni, R., et al. 2013, A\&A, 550, A21

Shenoy, S. S., Carey, S. J., Noriega-Crespo, A., et al. 2012, ApJ, submitted

Simpson, R. J., Nutter, D., \& Ward-Thompson, D. 2008, MNRAS, 391, 205

Swift, J. J., \& Beaumont, C. 2010, PASP, 122, 224

Thompson, M. A., Gibb, A. G., Hatchell, J. H., Wyrowski, F., \& Pillai, T. 2005, in The Dusty and Molecular Universe: A Prelude to Herschel and ALMA, 425

Thompson, M. A., Hatchell, J. H., Walsh, A. J., MacDonald, G. H., \& Millar, T. J. 2006, A\&A, 453, 1003

Veneziani, M., Elia, D., Noriega-Crespo, A., et al. 2013, A\&A, 549, A130

Wood, D. O. S., \& Churchwell, E. 1989, ApJS, 69, 831

Wright, E. L., Eisenhardt, P. R. M., Mainzer, A., et al. 2010, AJ, 140, 1868

Pages 15 to 21 are available in the electronic edition of the journal at http: //www . aanda. org 
M. T. Beltrán et al.: Hi-GAL sources in the G29.96-0.02 cloud

Table 1. Position, Hi-GAL fluxes, and MIPSGAL $24 \mu \mathrm{m}$ association for the sources detected by Herschel toward the G29.96-0.02 cloud.

\begin{tabular}{|c|c|c|c|c|c|c|c|c|c|c|}
\hline \# Id. ${ }^{a}$ & $\begin{array}{c}\alpha(\mathrm{J} 2000) \\
(\mathrm{h} \mathrm{m} \mathrm{s})\end{array}$ & $\begin{array}{c}\delta(\mathrm{J} 2000) \\
\left({ }^{\circ},{ }^{\prime \prime}\right)\end{array}$ & $\begin{array}{c}l \\
\left(^{\circ}\right)\end{array}$ & $\begin{array}{l}b \\
\left(^{\circ}\right)\end{array}$ & $\begin{array}{c}S_{70 \mu \mathrm{m}} \\
(\mathrm{Jy})\end{array}$ & $\begin{array}{c}S_{160 \mu \mathrm{m}} \\
(\mathrm{Jy})\end{array}$ & $\begin{array}{c}S_{250 \mu \mathrm{m}} \\
(\mathrm{Jy})\end{array}$ & $\begin{array}{c}S_{350 \mu \mathrm{m}} \\
(\mathrm{Jy})\end{array}$ & $\begin{array}{c}S_{500 \mu \mathrm{m}} \\
(\mathrm{Jy})\end{array}$ & $24 \mu \mathrm{m}$ MIPS \\
\hline 1 & 184606.05 & -24118.3 & 29.93 & -0.04 & $55 \pm 9$ & $58 \pm 8$ & $38 \pm 4$ & $25 \pm 3$ & $7.5 \pm 1.1$ & $\mathrm{~N}$ \\
\hline 2 & 184551.92 & -24223.8 & 29.89 & +0.00 & $99 \pm 12$ & $156 \pm 17$ & $95 \pm 11$ & $46 \pm 6$ & $17 \pm 2$ & Y \\
\hline 4 & 184611.67 & -23837.7 & 29.98 & -0.04 & $0.59 \pm 0.11$ & $24 \pm 5$ & $36 \pm 8$ & $24 \pm 5$ & $14 \pm 3$ & $\mathrm{~N}$ \\
\hline 5 & 184559.57 & -24310.4 & 29.89 & -0.03 & $0.70 \pm 0.13$ & $19 \pm 5$ & $14 \pm 3$ & $9.8 \pm 2.2$ & $2.8 \pm 0.7$ & $\mathrm{~N}$ \\
\hline 6 & 184557.49 & -24404.1 & 29.87 & -0.03 & $0.62 \pm 0.11$ & $15 \pm 4$ & $16 \pm 3$ & $10 \pm 2$ & $4.3 \pm 1.0$ & $\mathrm{~N}$ \\
\hline 7 & 184605.63 & -24432.8 & 29.88 & -0.06 & $0.72 \pm 0.13$ & $14 \pm 4$ & $12 \pm 3$ & $6.6 \pm 1.7$ & $2.2 \pm 0.6$ & $\mathrm{~N}$ \\
\hline 8 & 184606.38 & -24445.9 & 29.88 & -0.07 & $0.83 \pm 0.15$ & $12 \pm 1$ & $8.2 \pm 0.9$ & $4.8 \pm 0.7$ & $2.0 \pm 0.3$ & $\mathrm{~N}$ \\
\hline 9 & 184625.08 & -23752.2 & 30.02 & -0.08 & $0.34 \pm 0.06$ & $4.1 \pm 0.6$ & $6.2 \pm 0.8$ & $4.3 \pm 0.6$ & $1.7 \pm 0.3$ & $\mathrm{~N}$ \\
\hline 11 & 184626.19 & -23703.1 & 30.03 & -0.08 & $1.9 \pm 0.3$ & $6.5 \pm 0.8$ & $4.7 \pm 0.6$ & $2.0 \pm 0.3$ & $0.7 \pm 0.1$ & $\mathrm{~N}$ \\
\hline 12 & 184546.50 & -23314.1 & 30.01 & +0.09 & $0.36 \pm 0.07$ & $3.8 \pm 1.2$ & $9.5 \pm 1.2$ & $6.4 \pm 0.9$ & $2.8 \pm 0.4$ & $\mathrm{~N}$ \\
\hline 13 & 184548.53 & -23740.9 & 29.95 & +0.05 & $0.48 \pm 0.09$ & $8.9 \pm 1.3$ & $12 \pm 2$ & $7.2 \pm 1.1$ & $3.0 \pm 0.5$ & $\mathrm{~N}$ \\
\hline 14 & 184607.43 & -23406.0 & 30.04 & +0.01 & $3.8 \pm 0.43$ & $10 \pm 1$ & $8.6 \pm 1.0$ & $4.7 \pm 0.6$ & $1.8 \pm 0.2$ & $\mathrm{Y}$ \\
\hline 16 & 184641.10 & -23628.9 & 30.07 & -0.13 & $0.88 \pm 0.17$ & $5.5 \pm 0.6$ & $6.1 \pm 0.7$ & $3.8 \pm 0.5$ & $1.8 \pm 0.3$ & $\mathrm{~N}$ \\
\hline 17 & 184640.03 & -24149.3 & 29.99 & -0.17 & $2.7 \pm 0.3$ & $8.9 \pm 1.0$ & $11 \pm 1$ & $7.9 \pm 1.0$ & $4.0 \pm 0.6$ & $\mathrm{Y}$ \\
\hline 18 & 184641.04 & -23705.8 & 30.06 & -0.14 & $0.05 \pm 0.05$ & $2.8 \pm 1.8$ & $3.8 \pm 2.3$ & $2.6 \pm 1.6$ & $1.4 \pm 0.8$ & $\mathrm{~N}$ \\
\hline 19 & 184642.12 & -23728.4 & 30.06 & -0.14 & $0.71 \pm 0.13$ & $6.1 \pm 0.8$ & $5.7 \pm 0.7$ & $3.0 \pm 0.4$ & $1.2 \pm 0.2$ & $\mathrm{~N}$ \\
\hline 20 & 184640.78 & -23944.2 & 30.02 & -0.16 & $0.42 \pm 0.08$ & $3.5 \pm 0.4$ & $6.4 \pm 0.7$ & $4.5 \pm 0.6$ & $2.1 \pm 0.3$ & $\mathrm{~N}$ \\
\hline 21 & 184639.64 & -23514.3 & 30.08 & -0.12 & $0.85 \pm 0.15$ & $9.6 \pm 1.1$ & $6.0 \pm 0.7$ & $2.7 \pm 0.4$ & $1.1 \pm 0.2$ & $\mathrm{~N}$ \\
\hline 22 & 184642.42 & -24007.0 & 30.02 & -0.16 & $1.8 \pm 0.25$ & $7.7 \pm 0.9$ & $5.7 \pm 0.6$ & $2.5 \pm 0.3$ & $0.9 \pm 0.1$ & $\mathrm{~N}$ \\
\hline 24 & 184616.79 & -23456.4 & 30.05 & -0.03 & $0.85 \pm 0.11$ & $4.8 \pm 0.6$ & $5.3 \pm 0.6$ & $3.2 \pm 0.4$ & $0.7 \pm 0.1$ & $\mathrm{Y}$ \\
\hline 25 & 184631.69 & -23713.6 & 30.04 & -0.10 & $0.64 \pm 0.12$ & $6.9 \pm 0.8$ & $7.1 \pm 0.8$ & $4.0 \pm 0.5$ & $1.6 \pm 0.2$ & $\mathrm{~N}$ \\
\hline 26 & 184615.19 & -23427.6 & 30.05 & -0.02 & $0.48 \pm 0.09$ & $3.6 \pm 0.4$ & $6.1 \pm 0.7$ & $3.7 \pm 0.5$ & $1.7 \pm 0.2$ & $\mathrm{~N}$ \\
\hline 27 & 184640.23 & -23435.7 & 30.10 & -0.11 & $1.4 \pm 0.2$ & $7.2 \pm 1.0$ & $5.9 \pm 0.8$ & $3.0 \pm 0.4$ & $1.3 \pm 0.2$ & $\mathrm{~N}$ \\
\hline 31 & 184636.02 & -24240.3 & 29.97 & -0.16 & $0.38 \pm 0.07$ & $3.5 \pm 0.4$ & $6.9 \pm 0.8$ & $5.3 \pm 0.7$ & $2.9 \pm 0.4$ & $\mathrm{~N}$ \\
\hline 33 & 184543.88 & -23755.5 & 29.94 & +0.07 & $2.3 \pm 0.3$ & $7.3 \pm 0.821$ & $5.6 \pm 0.6$ & $2.5 \pm 0.3$ & $0.7 \pm 0.1$ & Y \\
\hline 34 & 184627.23 & -23406.7 & 30.08 & -0.06 & $0.71 \pm 0.13$ & $5.8 \pm 0.7$ & $5.3 \pm 0.6$ & $2.8 \pm 0.4$ & $1.0 \pm 0.1$ & $\mathrm{~N}$ \\
\hline 35 & 184549.20 & -23518.6 & 29.99 & +0.07 & $1.8 \pm 0.2$ & $6.7 \pm 0.7$ & $5.1 \pm 0.6$ & $2.2 \pm 0.3$ & $0.8 \pm 0.1$ & $\mathrm{~N}$ \\
\hline 37 & 184547.36 & -23605.6 & 29.97 & +0.07 & $1.1 \pm 0.2$ & $6.3 \pm 1.1$ & $5.0 \pm 0.9$ & $2.9 \pm 0.5$ & $1.2 \pm 0.2$ & Y \\
\hline 38 & 184652.32 & -23916.4 & 30.05 & -0.20 & $2.0 \pm 0.3$ & $5.2 \pm 0.6$ & $3.8 \pm 0.4$ & $1.8 \pm 0.2$ & $0.6 \pm 0.1$ & $\mathrm{~N}$ \\
\hline 40 & 184548.46 & -23447.7 & 29.99 & +0.08 & $2.2 \pm 0.4$ & $5.1 \pm 0.9$ & $3.8 \pm 0.6$ & $1.9 \pm 0.3$ & $0.7 \pm 0.1$ & $\mathrm{~N}$ \\
\hline 42 & 184622.46 & -23406.8 & 30.07 & -0.05 & $2.1 \pm 0.3$ & $5.5 \pm 0.6$ & $3.9 \pm 0.5$ & $1.8 \pm 0.2$ & $0.6 \pm 0.1$ & $\mathrm{Y}$ \\
\hline 44 & 184619.25 & -24640.4 & 29.88 & -0.13 & $3.4 \pm 0.5$ & $7.3 \pm 0.9$ & $5.0 \pm 0.6$ & $3.2 \pm 0.4$ & $1.4 \pm 0.2$ & $\mathrm{~N}$ \\
\hline 45 & 184559.56 & -24942.1 & 29.79 & -0.08 & $0.54 \pm 0.10$ & $6.1 \pm 0.7$ & $7.7 \pm 0.9$ & $4.5 \pm 0.6$ & $1.8 \pm 0.2$ & $\mathrm{~N}$ \\
\hline 48 & 184616.81 & -23347.2 & 30.06 & -0.02 & $3.3 \pm 0.4$ & $5.8 \pm 0.6$ & $3.5 \pm 0.4$ & $1.6 \pm 0.2$ & $0.5 \pm 0.1$ & $\mathrm{~N}$ \\
\hline 49 & 184655.28 & -23633.1 & 30.10 & -0.19 & $1.8 \pm 0.3$ & $5.1 \pm 0.6$ & $4.1 \pm 0.5$ & $2.0 \pm 0.3$ & $0.8 \pm 0.1$ & $\mathrm{~N}$ \\
\hline 54 & 184628.85 & -23114.1 & 30.12 & -0.05 & $2.0 \pm 0.2$ & $3.5 \pm 0.4$ & $2.7 \pm 0.3$ & $1.1 \pm 0.1$ & $0.2 \pm 0.03$ & $\mathrm{~N}$ \\
\hline 55 & 184658.48 & -23522.8 & 30.12 & -0.19 & $0.67 \pm 0.12$ & $4.1 \pm 0.5$ & $4.4 \pm 0.5$ & $2.6 \pm 0.3$ & $1.2 \pm 0.2$ & $\mathrm{~N}$ \\
\hline 61 & 184649.82 & -23342.6 & 30.13 & -0.14 & $0.45 \pm 0.11$ & $6.7 \pm 0.7$ & $5.1 \pm 0.6$ & $2.6 \pm 0.3$ & $1.0 \pm 0.1$ & $\mathrm{~N}$ \\
\hline 62 & 184628.18 & -25000.2 & 29.84 & -0.19 & $1.0 \pm 0.1$ & $4.0 \pm 0.4$ & $3.5 \pm 0.4$ & $1.9 \pm 0.2$ & $0.9 \pm 0.1$ & $\mathrm{~N}$ \\
\hline 64 & 184637.41 & -24528.8 & 29.93 & -0.19 & $1.1 \pm 0.1$ & $5.0 \pm 0.6$ & $4.6 \pm 0.5$ & $2.6 \pm 0.3$ & $1.0 \pm 0.1$ & Y \\
\hline 65 & 184556.00 & -24946.6 & 29.79 & -0.07 & $2.3 \pm 0.3$ & $6.4 \pm 0.7$ & $4.4 \pm 0.5$ & $2.0 \pm 0.3$ & $0.7 \pm 0.1$ & Y \\
\hline 66 & 184628.51 & -24915.7 & 29.86 & -0.18 & $1.4 \pm 0.2$ & $3.9 \pm 0.5$ & $3.3 \pm 0.4$ & $1.8 \pm 0.2$ & $0.8 \pm 0.1$ & $\mathrm{~N}$ \\
\hline 69 & 184617.94 & -25146.3 & 29.80 & -0.16 & $6.8 \pm 0.8$ & $9.6 \pm 1.1$ & $5.5 \pm 0.6$ & $2.5 \pm 0.3$ & $0.9 \pm 0.1$ & Y \\
\hline 70 & 184627.79 & -25134.9 & 29.82 & -0.20 & $0.32 \pm 0.06$ & $3.1 \pm 0.4$ & $7.7 \pm 0.9$ & $6.2 \pm 0.8$ & $3.3 \pm 0.5$ & $\mathrm{~N}$ \\
\hline 73 & 184627.25 & -25050.9 & 29.83 & -0.19 & $0.42 \pm 0.08$ & $2.1 \pm 0.3$ & $4.7 \pm 0.5$ & $3.5 \pm 0.5$ & $2.0 \pm 0.3$ & $\mathrm{~N}$ \\
\hline 74 & 184553.61 & -24927.9 & 29.79 & -0.06 & $0.28 \pm 0.19$ & $7.5 \pm 0.9$ & $5.5 \pm 0.6$ & $2.6 \pm 0.3$ & $0.9 \pm 0.1$ & $\mathrm{~N}$ \\
\hline 75 & 184629.85 & -24518.8 & 29.92 & -0.16 & $0.39 \pm 0.13$ & $4.3 \pm 0.5$ & $3.1 \pm 0.3$ & $1.4 \pm 0.2$ & $0.4 \pm 0.1$ & $\mathrm{~N}$ \\
\hline 77 & 184623.22 & -25044.3 & 29.82 & -0.18 & $1.9 \pm 0.3$ & $4.0 \pm 0.5$ & $3.3 \pm 0.4$ & $1.6 \pm 0.2$ & $0.6 \pm 0.1$ & $\mathrm{~N}$ \\
\hline 79 & 184612.13 & -25147.1 & 29.79 & -0.14 & $1.7 \pm 0.2$ & $8.1 \pm 0.9$ & $5.9 \pm 0.7$ & $2.7 \pm 0.4$ & $0.7 \pm 0.1$ & $\mathrm{Y}$ \\
\hline 81 & 184640.58 & -24545.8 & 29.93 & -0.20 & $2.8 \pm 0.4$ & $5.5 \pm 0.7$ & $3.8 \pm 0.5$ & $2.0 \pm 0.3$ & $0.8 \pm 0.1$ & Y \\
\hline 83 & 184700.90 & -23554.5 & 30.12 & -0.20 & $0.63 \pm 0.11$ & $2.8 \pm 0.3$ & $3.1 \pm 0.3$ & $1.9 \pm 0.3$ & $0.8 \pm 0.1$ & $\mathrm{~N}$ \\
\hline 86 & 184636.87 & -24622.0 & 29.91 & -0.19 & $0.13 \pm 0.05$ & $2.0 \pm 0.4$ & $2.1 \pm 0.4$ & $1.1 \pm 0.2$ & $0.3 \pm 0.1$ & $\mathrm{~N}$ \\
\hline
\end{tabular}

Notes. ${ }^{(a)}$ The identification numbers of the sources are not sequential. 
Table 1. continued.

\begin{tabular}{|c|c|c|c|c|c|c|c|c|c|c|}
\hline \# Id. ${ }^{a}$ & $\begin{array}{c}\alpha(\mathrm{J} 2000) \\
(\mathrm{h} \mathrm{m} \mathrm{s})\end{array}$ & $\begin{array}{c}\delta(\mathrm{J} 2000) \\
\left({ }^{\circ \prime \prime \prime \prime}\right)\end{array}$ & $\begin{array}{c}l \\
\left(^{\circ}\right)\end{array}$ & $\begin{array}{l}b \\
\left(^{\circ}\right)\end{array}$ & $\begin{array}{c}S_{70 \mu \mathrm{m}} \\
(\mathrm{Jy})\end{array}$ & $\begin{array}{c}S_{160 \mu \mathrm{m}} \\
(\mathrm{Jy})\end{array}$ & $\begin{array}{c}S_{250 \mu \mathrm{m}} \\
(\mathrm{Jy})\end{array}$ & $\begin{array}{c}S_{350 \mu \mathrm{m}} \\
(\mathrm{Jy})\end{array}$ & $\begin{array}{c}S_{500 \mu \mathrm{m}} \\
(\mathrm{Jy})\end{array}$ & $24 \mu \mathrm{m}$ MIPS \\
\hline 87 & 184634.97 & -24554.8 & 29.92 & -0.18 & $0.89 \pm 0.14$ & $3.8 \pm 0.5$ & $3.3 \pm 0.4$ & $1.6 \pm 0.2$ & $0.5 \pm 0.1$ & $\mathrm{~N}$ \\
\hline 88 & 184646.84 & -24332.0 & 29.98 & -0.21 & $0.89 \pm 0.16$ & $5.8 \pm 0.6$ & $4.6 \pm 0.5$ & $2.3 \pm 0.3$ & $0.7 \pm 0.1$ & $\mathrm{~N}$ \\
\hline 90 & 184627.24 & -24728.4 & 29.88 & -0.16 & $1.4 \pm 0.2$ & $4.3 \pm 0.6$ & $3.8 \pm 0.5$ & $2.0 \pm 0.3$ & $0.7 \pm 0.1$ & Y \\
\hline 98 & 184535.30 & -23833.8 & 29.91 & +0.10 & $1.6 \pm 0.2$ & $11 \pm 1$ & $10 \pm 1$ & $6.7 \pm 0.9$ & $3.4 \pm 0.5$ & $\mathrm{~N}$ \\
\hline 99 & 184537.76 & -23556.5 & 29.96 & +0.11 & $2.7 \pm 0.3$ & $3.5 \pm 0.4$ & $3.2 \pm 0.4$ & $2.0 \pm 0.3$ & $1.1 \pm 0.1$ & $\mathrm{~N}$ \\
\hline 100 & 184644.22 & -24344.2 & 29.97 & -0.20 & $2.6 \pm 0.5$ & $3.6 \pm 0.6$ & $2.0 \pm 0.4$ & $0.7 \pm 0.1$ & $0.09 \pm 0.02$ & $\mathrm{~N}$ \\
\hline 104 & 184617.83 & -22950.3 & 30.12 & +0.00 & $0.70 \pm 0.11$ & $5.3 \pm 0.6$ & $3.8 \pm 0.4$ & $1.9 \pm 0.2$ & $0.6 \pm 0.1$ & Y \\
\hline 109 & 184536.99 & -24125.1 & 29.87 & +0.07 & $0.97 \pm 0.18$ & $4.6 \pm 0.6$ & $3.5 \pm 0.4$ & $1.6 \pm 0.2$ & $0.6 \pm 0.1$ & $\mathrm{~N}$ \\
\hline 111 & 184535.83 & -24000.6 & 29.89 & +0.08 & $0.70 \pm 0.13$ & $3.7 \pm 0.6$ & $3.5 \pm 0.6$ & $1.9 \pm 0.3$ & $0.8 \pm 0.1$ & $\mathrm{~N}$ \\
\hline 113 & 184531.58 & -23933.8 & 29.89 & +0.10 & $0.81 \pm 0.15$ & $2.0 \pm 0.7$ & $2.4 \pm 0.8$ & $1.8 \pm 0.6$ & $0.8 \pm 0.3$ & $\mathrm{~N}$ \\
\hline 122 & 184609.87 & -24108.1 & 29.94 & -0.05 & $187 \pm 21$ & $219 \pm 25$ & $117 \pm 13$ & $44 \pm 6$ & $15 \pm 2$ & Y \\
\hline 123 & 184605.00 & -24223.6 & 29.91 & -0.04 & $46 \pm 8$ & $210 \pm 24$ & $171 \pm 19$ & $97 \pm 13$ & $38 \pm 5$ & Y \\
\hline 124 & 184608.76 & -24201.8 & 29.93 & -0.05 & $39 \pm 12$ & $77 \pm 22$ & $57 \pm 16$ & $28 \pm 8$ & $6 \pm 2$ & Y \\
\hline 125 & 184611.87 & -24130.7 & 29.94 & -0.06 & $184 \pm 27$ & $162 \pm 22$ & $79 \pm 11$ & $40 \pm 6$ & $20 \pm 3$ & Y \\
\hline 126 & 184612.87 & -23858.3 & 29.98 & -0.05 & $36 \pm 4$ & $128 \pm 14$ & $111 \pm 12$ & $66 \pm 9$ & $29 \pm 4$ & Y \\
\hline 127 & 184600.41 & -24114.9 & 29.92 & -0.02 & $122 \pm 15$ & $150 \pm 17$ & $85 \pm 10$ & $37 \pm 5$ & $12 \pm 2$ & $\mathrm{~N}$ \\
\hline 129 & 184559.01 & -24110.1 & 29.92 & -0.01 & $25 \pm 4$ & $63 \pm 9$ & $62 \pm 9$ & $34 \pm 5$ & $15 \pm 2$ & $\mathrm{~N}$ \\
\hline 130 & 184612.92 & -23929.6 & 29.97 & -0.05 & $0.65 \pm 0.12$ & $64 \pm 7$ & $92 \pm 10$ & $56 \pm 7$ & $25 \pm 4$ & $\mathrm{~N}$ \\
\hline 131 & 184606.45 & -23749.2 & 29.98 & -0.01 & $0.65 \pm 0.12$ & $36 \pm 2$ & $39 \pm 29$ & $27 \pm 20$ & $11 \pm 8$ & $\mathrm{~N}$ \\
\hline 132 & 184610.96 & -24328.2 & 29.91 & -0.07 & $52 \pm 8$ & $37 \pm 5$ & $19 \pm 3$ & $7.9 \pm 1.2$ & $1.7 \pm 0.3$ & Y \\
\hline 133 & 184613.16 & -23635.6 & 30.01 & -0.03 & $0.68 \pm 0.12$ & $33 \pm 8$ & $28 \pm 6$ & $17 \pm 3.9$ & $6.9 \pm 1.7$ & $\mathrm{~N}$ \\
\hline 134 & 184558.73 & -24032.7 & 29.93 & -0.01 & $0.58 \pm 0.10$ & $28 \pm 3$ & $42 \pm 5$ & $28 \pm 3.6$ & $15 \pm 2$ & $\mathrm{~N}$ \\
\hline 135 & 184613.04 & -24337.9 & 29.91 & -0.08 & $26 \pm 21$ & $8.4 \pm 7$ & $3.0 \pm 2.5$ & $1.4 \pm 1.1$ & $0.09 \pm 0.02$ & Y \\
\hline 136 & 184613.66 & -23729.1 & 30.00 & -0.04 & $0.51 \pm 0.09$ & $12 \pm 3$ & $14 \pm 2$ & $8.6 \pm 1.6$ & $2.9 \pm 0.6$ & $\mathrm{~N}$ \\
\hline 137 & 184555.11 & -23919.5 & 29.94 & +0.02 & $18 \pm 2$ & $61 \pm 7$ & $46 \pm 5$ & $22 \pm 2.8$ & $6.9 \pm 1.0$ & Y \\
\hline 138 & 184617.21 & -23817.4 & 30.00 & -0.06 & $7.4 \pm 1.1$ & $15 \pm 2$ & $12 \pm 2$ & $6.0 \pm 0.9$ & $0.88 \pm 0.21$ & Y \\
\hline 139 & 184623.72 & -24101.0 & 29.97 & -0.10 & $23 \pm 3$ & $23 \pm 3$ & $15 \pm 2$ & $7.2 \pm 0.9$ & $1.7 \pm 0.2$ & Y \\
\hline 141 & 184607.15 & -24458.5 & 29.88 & -0.07 & $5.5 \pm 2.7$ & $5.5 \pm 2.6$ & $2.6 \pm 1.2$ & $1.1 \pm 0.6$ & $0.19 \pm 0.16$ & $\mathrm{~N}$ \\
\hline 142 & 184552.10 & -24346.4 & 29.87 & -0.01 & $0.65 \pm 0.12$ & $20 \pm 3$ & $21 \pm 2$ & $9.8 \pm 1.3$ & $3.0 \pm 0.4$ & $\mathrm{~N}$ \\
\hline 143 & 184617.64 & -23806.9 & 30.00 & -0.06 & $2.7 \pm 1.8$ & $1.8 \pm 1.3$ & $0.89 \pm 0.79$ & $0.10 \pm 0.02$ & $0.09 \pm 0.02$ & $\mathrm{Y}$ \\
\hline 144 & 184622.17 & -23704.6 & 30.02 & -0.07 & $11 \pm 2$ & $9.8 \pm 2.2$ & $7.2 \pm 1.8$ & $3.7 \pm 1.1$ & $1.4 \pm 0.5$ & $\mathrm{~N}$ \\
\hline 147 & 184620.96 & -23857.5 & 30.00 & -0.08 & $16 \pm 2$ & $11 \pm 1$ & $5.9 \pm 0.7$ & $1.8 \pm 0.2$ & $0.33 \pm 0.06$ & Y \\
\hline 148 & 184554.33 & -23821.9 & 29.95 & +0.03 & $2.6 \pm 0.4$ & $30 \pm 3$ & $27 \pm 3$ & $14 \pm 2$ & $6.6 \pm 0.9$ & Y \\
\hline 149 & 184553.99 & -23852.9 & 29.94 & +0.02 & $3.6 \pm 0.5$ & $30 \pm 4$ & $25 \pm 3$ & $13 \pm 2$ & $5.4 \pm 0.8$ & $\mathrm{~N}$ \\
\hline 150 & 184555.02 & -24559.7 & 29.84 & -0.03 & $1.9 \pm 0.4$ & $30 \pm 4$ & $31 \pm 4$ & $17 \pm 2$ & $5.7 \pm 0.8$ & $\mathrm{~N}$ \\
\hline 151 & 184554.60 & -24542.7 & 29.84 & -0.03 & $0.69 \pm 0.13$ & $11 \pm 2$ & $13 \pm 2$ & $9.3 \pm 1.3$ & $5.1 \pm 0.8$ & $\mathrm{~N}$ \\
\hline 152 & 184603.88 & -24831.2 & 29.82 & -0.09 & $4.2 \pm 0.8$ & $32 \pm 4$ & $22 \pm 2$ & $10 \pm 1$ & $3.0 \pm 0.4$ & $\mathrm{Y}$ \\
\hline 153 & 184601.99 & -23529.2 & 30.01 & +0.02 & $23 \pm 3$ & $23 \pm 3$ & $13 \pm 1$ & $5.2 \pm 0.7$ & $1.5 \pm 0.2$ & $\mathrm{Y}$ \\
\hline 155 & 184546.45 & -24247.2 & 29.87 & +0.02 & $1.4 \pm 0.7$ & $11 \pm 2$ & $11 \pm 2$ & $9.2 \pm 2.0$ & $4.2 \pm 0.9$ & $\mathrm{~N}$ \\
\hline 159 & 184547.89 & -24439.4 & 29.85 & +0.00 & $2.8 \pm 0.4$ & $27 \pm 3$ & $30 \pm 3$ & $19 \pm 2$ & $7.8 \pm 1.1$ & $\mathrm{Y}$ \\
\hline 160 & 184555.86 & -23723.3 & 29.97 & +0.03 & $10 \pm 1$ & $17 \pm 2$ & $11 \pm 1$ & $4.0 \pm 0.5$ & $0.57 \pm 0.10$ & Y \\
\hline 161 & 184553.42 & -24527.1 & 29.85 & -0.02 & $0.87 \pm 0.16$ & $11 \pm 2$ & $9.5 \pm 1.3$ & $4.4 \pm 0.7$ & $0.81 \pm 0.17$ & $\mathrm{~N}$ \\
\hline 162 & 184615.53 & -24418.5 & 29.90 & -0.10 & $4.8 \pm 1.4$ & $9.8 \pm 1$ & $9.6 \pm 1.2$ & $5.7 \pm 0.8$ & $2.8 \pm 0.4$ & $\mathrm{~N}$ \\
\hline 163 & 184601.89 & -24700.7 & 29.84 & -0.07 & $0.65 \pm 0.12$ & $10 \pm 8$ & $10 \pm 8$ & $5.8 \pm 4.4$ & $2.4 \pm 1.8$ & $\mathrm{~N}$ \\
\hline 164 & 184551.09 & -24430.4 & 29.86 & -0.01 & $0.65 \pm 0.12$ & $6.1 \pm 1.5$ & $7.2 \pm 1.7$ & $4.7 \pm 1.2$ & $2.8 \pm 0.7$ & $\mathrm{~N}$ \\
\hline 165 & 184608.89 & -23516.5 & 30.03 & -0.00 & $5.7 \pm 0.7$ & $12 \pm 1$ & $12 \pm 1$ & $7.0 \pm 0.9$ & $2.4 \pm 0.3$ & $\mathrm{Y}$ \\
\hline 167 & 184611.40 & -24824.1 & 29.84 & -0.11 & $10 \pm 1$ & $12 \pm 2$ & $7.9 \pm 1.0$ & $3.2 \pm 0.5$ & $1.0 \pm 0.2$ & $\mathrm{Y}$ \\
\hline 168 & 184601.66 & -24749.7 & 29.83 & -0.07 & $3.5 \pm 0.6$ & $22 \pm 2$ & $17 \pm 2$ & $9.0 \pm 1.2$ & $3.3 \pm 0.5$ & $\mathrm{Y}$ \\
\hline 169 & 184544.69 & -24224.9 & 29.87 & +0.03 & $5.0 \pm 0.7$ & $9.6 \pm 1.1$ & $7.0 \pm 0.8$ & $3.7 \pm 0.6$ & $2.5 \pm 0.4$ & $\mathrm{Y}$ \\
\hline 170 & 184629.94 & -23627.1 & 30.05 & -0.09 & $4.5 \pm 0.6$ & $16 \pm 2$ & $12 \pm 1$ & $6.0 \pm 0.8$ & $2.2 \pm 0.3$ & Y \\
\hline 171 & 184559.89 & -24725.5 & 29.83 & -0.06 & $0.59 \pm 0.11$ & $13 \pm 1$ & $20 \pm 2$ & $12 \pm 2$ & $4.4 \pm 0.6$ & $\mathrm{~N}$ \\
\hline 172 & 184542.87 & -24253.5 & 29.86 & +0.03 & $4.3 \pm 0.6$ & $21 \pm 3$ & $27 \pm 3$ & $19 \pm 3$ & $12 \pm 2$ & Y \\
\hline 175 & 184631.77 & -23933.6 & 30.01 & -0.12 & $0.56 \pm 0.10$ & $7.6 \pm 0.9$ & $12 \pm 1$ & $9.7 \pm 1.3$ & $5.8 \pm 0.8$ & $\mathrm{~N}$ \\
\hline 177 & 184605.18 & -23009.6 & 30.09 & +0.05 & $11 \pm 1$ & $26 \pm 3$ & $18 \pm 2$ & $8.9 \pm 1.2$ & $3.6 \pm 0.5$ & $\mathrm{Y}$ \\
\hline
\end{tabular}


Table 1. continued.

\begin{tabular}{|c|c|c|c|c|c|c|c|c|c|c|}
\hline \# Id. ${ }^{a}$ & $\begin{array}{c}\alpha(\mathrm{J} 2000) \\
(\mathrm{h} \mathrm{m} \mathrm{s})\end{array}$ & $\begin{array}{c}\delta(\mathrm{J} 2000) \\
\left({ }^{\circ}, \prime \prime\right)\end{array}$ & $\begin{array}{c}l \\
\left({ }^{\circ}\right)\end{array}$ & $\begin{array}{c}b \\
\left({ }^{\circ}\right)\end{array}$ & $\begin{array}{c}S_{70 \mu \mathrm{m}} \\
(\mathrm{Jy})\end{array}$ & $\begin{array}{c}S_{160 \mu \mathrm{m}} \\
(\mathrm{Jy})\end{array}$ & $\begin{array}{c}S_{250 \mu \mathrm{m}} \\
(\mathrm{Jy})\end{array}$ & $\begin{array}{c}S_{350 \mu \mathrm{m}} \\
(\mathrm{Jy})\end{array}$ & $\begin{array}{c}S_{500 \mu \mathrm{m}} \\
(\mathrm{Jy})\end{array}$ & $24 \mu \mathrm{m}$ MIPS \\
\hline 178 & 184647.22 & -23936.4 & 30.03 & -0.18 & $3.7 \pm 0.5$ & $17 \pm 2$ & $14 \pm 2$ & $6.8 \pm 0.9$ & $2.9 \pm 0.4$ & $\mathrm{Y}$ \\
\hline 179 & 184642.73 & -23541.6 & 30.08 & -0.13 & $6.1 \pm 0.7$ & $6.5 \pm 0.7$ & $3.6 \pm 0.4$ & $1.7 \pm 0.2$ & $0.71 \pm 0.11$ & Y \\
\hline 180 & 184650.21 & -24136.5 & 30.01 & -0.21 & $7.6 \pm 0.8$ & $14 \pm 2$ & $8.5 \pm 0.9$ & $3.8 \pm 0.5$ & $1.3 \pm 0.2$ & Y \\
\hline 182 & 184556.20 & -24713.3 & 29.82 & -0.05 & $1.6 \pm 0.4$ & $27 \pm 3$ & $23 \pm 3$ & $12 \pm 2$ & $4.2 \pm 0.6$ & $\mathrm{Y}$ \\
\hline 184 & 184544.88 & -24331.6 & 29.86 & +0.02 & $1.6 \pm 0.5$ & $14 \pm 2$ & $17 \pm 2$ & $12 \pm 2$ & $7.1 \pm 1.1$ & Y \\
\hline 185 & 184623.02 & -24349.3 & 29.93 & -0.12 & $4.3 \pm 0.6$ & $7.5 \pm 0.9$ & $4.8 \pm 0.5$ & $1.9 \pm 0.2$ & $0.64 \pm 0.09$ & $\mathrm{Y}$ \\
\hline 186 & 184615.38 & -24944.0 & 29.82 & -0.14 & $4.0 \pm 0.6$ & $8.9 \pm 1.0$ & $4.6 \pm 0.5$ & $1.9 \pm 0.2$ & $0.77 \pm 0.11$ & $\mathrm{Y}$ \\
\hline 187 & 184549.75 & -23248.2 & 30.03 & +0.09 & $5.2 \pm 0.6$ & $19 \pm 2$ & $14 \pm 2$ & $5.6 \pm 0.7$ & $2.2 \pm 0.3$ & Y \\
\hline 188 & 184646.27 & -23620.0 & 30.08 & -0.15 & $2.1 \pm 0.3$ & $6.1 \pm 0.7$ & $4.0 \pm 0.5$ & $1.7 \pm 0.2$ & $0.62 \pm 0.09$ & $\mathrm{Y}$ \\
\hline 189 & 184646.57 & -23542.9 & 30.09 & -0.15 & $6.6 \pm 0.8$ & $8.7 \pm 1.1$ & $5.5 \pm 0.7$ & $2.8 \pm 0.4$ & $1.3 \pm 0.2$ & $\mathrm{Y}$ \\
\hline 191 & 184648.73 & -24017.9 & 30.03 & -0.19 & $3.8 \pm 0.4$ & $12 \pm 1$ & $8.8 \pm 1.0$ & $4.0 \pm 0.5$ & $1.3 \pm 0.2$ & $\mathrm{~N}$ \\
\hline 192 & 184642.78 & -23849.1 & 30.04 & -0.16 & $0.95 \pm 0.16$ & $9.0 \pm 1.1$ & $6.6 \pm 0.8$ & $2.9 \pm 0.4$ & $0.79 \pm 0.13$ & Y \\
\hline 193 & 184550.00 & -23049.7 & 30.06 & +0.10 & $0.54 \pm 0.10$ & $10 \pm 2$ & $13 \pm 3$ & $9.8 \pm 2.0$ & $4.7 \pm 1.0$ & $\mathrm{~N}$ \\
\hline 194 & 184545.93 & -23636.7 & 29.96 & +0.08 & $15 \pm 2$ & $18 \pm 2$ & $12 \pm 1$ & $5.8 \pm 0.7$ & $2.6 \pm 0.4$ & $\mathrm{Y}$ \\
\hline 195 & 184631.55 & -23234.5 & 30.11 & -0.07 & $0.30 \pm 0.08$ & $5.1 \pm 0.6$ & $7.7 \pm 0.9$ & $6.4 \pm 0.9$ & $3.3 \pm 0.5$ & Y \\
\hline 196 & 184547.56 & -23722.7 & 29.95 & +0.06 & $3.1 \pm 0.4$ & $15 \pm 2$ & $11 \pm 1$ & $5.6 \pm 0.7$ & $2.2 \pm 0.3$ & $\mathrm{Y}$ \\
\hline 197 & 184652.33 & -24002.5 & 30.04 & -0.20 & $9.9 \pm 1.2$ & $12 \pm 1$ & $6.7 \pm 0.8$ & $2.3 \pm 0.3$ & $0.31 \pm 0.06$ & Y \\
\hline 198 & 184550.16 & -23501.3 & 29.99 & +0.07 & $9.3 \pm 1.0$ & $14 \pm 2$ & $7.0 \pm 0.8$ & $2.8 \pm 0.4$ & $0.85 \pm 0.12$ & $\mathrm{Y}$ \\
\hline 199 & 184648.81 & -24117.3 & 30.01 & -0.20 & $12 \pm 1$ & $19 \pm 2$ & $10 \pm 1$ & $3.9 \pm 0.5$ & $1.0 \pm 0.1$ & Y \\
\hline 200 & 184610.48 & -24623.5 & 29.86 & -0.09 & $0.67 \pm 0.12$ & $101 \pm 1$ & $9.7 \pm 1.1$ & $5.2 \pm 0.7$ & $2.0 \pm 0.3$ & $\mathrm{~N}$ \\
\hline 201 & 184633.82 & -23423.3 & 30.09 & -0.09 & $5.4 \pm 0.6$ & $8.9 \pm 1.0$ & $5.1 \pm 0.6$ & $2.1 \pm 0.3$ & $0.77 \pm 0.11$ & $\mathrm{Y}$ \\
\hline 202 & 184645.27 & -2 3916.9 & 30.04 & -0.17 & $2.3 \pm 0.4$ & $7.2 \pm 1.1$ & $4.4 \pm 0.7$ & $1.7 \pm 0.3$ & $0.46 \pm 0.09$ & $\mathrm{Y}$ \\
\hline 203 & 184545.77 & -23900.4 & 29.93 & +0.05 & $1.5 \pm 0.2$ & $12 \pm 1$ & $12 \pm 1$ & $7.1 \pm 0.9$ & $3.0 \pm 0.4$ & $\mathrm{Y}$ \\
\hline 205 & 184652.16 & -24207.0 & 30.01 & -0.22 & $6.9 \pm 0.8$ & $13 \pm 1$ & $8.5 \pm 0.9$ & $3.7 \pm 0.5$ & $1.2 \pm 0.2$ & $\mathrm{Y}$ \\
\hline 207 & 184614.92 & -25017.6 & 29.81 & -0.14 & $13 \pm 2$ & $9.2 \pm 1.3$ & $4.6 \pm 0.6$ & $1.8 \pm 0.3$ & $0.61 \pm 0.10$ & $\mathrm{Y}$ \\
\hline 208 & 184559.55 & -24826.6 & 29.81 & -0.07 & $0.89 \pm 0.16$ & $6.6 \pm 0.8$ & $4.7 \pm 0.5$ & $1.8 \pm 0.3$ & $0.42 \pm 0.08$ & $\mathrm{~N}$ \\
\hline 209 & 184542.44 & -23126.2 & 30.03 & +0.12 & $0.52 \pm 0.09$ & $14 \pm 3$ & $23 \pm 5$ & $16 \pm 4$ & $7.8 \pm 2.0$ & $\mathrm{~N}$ \\
\hline 210 & 184649.13 & -23804.4 & 30.06 & -0.17 & $9.5 \pm 1.1$ & $9.3 \pm 1.0$ & $5.0 \pm 0.6$ & $2.0 \pm 0.3$ & $0.47 \pm 0.07$ & $\mathrm{Y}$ \\
\hline 212 & 184550.40 & -24758.4 & 29.80 & -0.03 & $1.3 \pm 0.2$ & $10 \pm 1$ & $12 \pm 1$ & $8 \pm 1$ & $3.6 \pm 0.5$ & $\mathrm{~N}$ \\
\hline 213 & 184651.47 & -23802.6 & 30.07 & -0.18 & $6.2 \pm 0.7$ & $11 \pm 1$ & $6.8 \pm 0.8$ & $2.7 \pm 0.4$ & $0.90 \pm 0.13$ & $\mathrm{Y}$ \\
\hline 214 & 184543.98 & -24511.7 & 29.83 & +0.01 & $5.3 \pm 0.6$ & $21 \pm 2$ & $23 \pm 3$ & $13 \pm 2$ & $5.0 \pm 0.7$ & $\mathrm{~N}$ \\
\hline 215 & 184556.64 & -23445.2 & 30.01 & +0.05 & $1.1 \pm 0.1$ & $7.5 \pm 0.9$ & $9.0 \pm 1.0$ & $5.8 \pm 0.8$ & $2.7 \pm 0.4$ & Y \\
\hline 217 & 184553.82 & -24655.7 & 29.82 & -0.04 & $0.79 \pm 0.14$ & $13 \pm 1$ & $10 \pm 1$ & $4.8 \pm 0.6$ & $1.9 \pm 0.3$ & $\mathrm{~N}$ \\
\hline 219 & 184601.96 & -23047.6 & 30.08 & +0.06 & $0.29 \pm 0.21$ & $6.9 \pm 0.9$ & $5.2 \pm 0.7$ & $2.5 \pm 0.4$ & $0.69 \pm 0.12$ & $\mathrm{Y}$ \\
\hline 220 & 184614.12 & -23210.8 & 30.08 & +0.00 & $4.0 \pm 0.4$ & $10 \pm 1$ & $7.0 \pm 0.8$ & $3.1 \pm 0.4$ & $1.2 \pm 0.2$ & $\mathrm{Y}$ \\
\hline 221 & 184610.14 & -25121.1 & 29.79 & -0.13 & $3.3 \pm 0.4$ & $12 \pm 1$ & $7.7 \pm 0.9$ & $3.2 \pm 0.4$ & $0.97 \pm 0.14$ & Y \\
\hline 228 & 184550.23 & -24825.2 & 29.80 & -0.03 & $0.49 \pm 0.15$ & $10 \pm 1$ & $9.9 \pm 1.1$ & $4.8 \pm 0.6$ & $1.6 \pm 0.2$ & Y \\
\hline 232 & 184535.61 & -23904.1 & 29.91 & +0.09 & $2.0 \pm 0.3$ & $11 \pm 1$ & $9.2 \pm 1.0$ & $4.4 \pm 0.6$ & $1.7 \pm 0.2$ & $\mathrm{~N}$ \\
\hline 242 & 184603.84 & -23921.2 & 29.96 & -0.02 & $7235 \pm 809$ & $1810 \pm 202$ & $498 \pm 56$ & $348 \pm 45$ & $105 \pm 15$ & Y \\
\hline 243 & 184559.45 & -24505.8 & 29.86 & -0.04 & $552 \pm 62$ & $228 \pm 25$ & $115 \pm 13$ & $56 \pm 7$ & $20 \pm 3$ & $\mathrm{Y}$ \\
\hline 245 & 184611.25 & -24156.2 & 29.93 & -0.06 & $605 \pm 68$ & $338 \pm 38$ & $184 \pm 21$ & $90 \pm 12$ & $28 \pm 4$ & $\mathrm{Y}$ \\
\hline 247 & 184617.08 & -23643.5 & 30.02 & -0.05 & $601 \pm 67$ & $280 \pm 31$ & $142 \pm 16$ & $65 \pm 8$ & $25 \pm 3$ & Y \\
\hline 251 & 184554.67 & -24253.2 & 29.86 & -0.01 & $222 \pm 25$ & $76 \pm 8$ & $40 \pm 4$ & $15 \pm 2$ & $3.2 \pm 0.5$ & Y \\
\hline 253 & 184601.75 & -24527.7 & 29.86 & -0.05 & $352 \pm 39$ & $186 \pm 21$ & $91 \pm 10$ & $36 \pm 5$ & $13 \pm 2$ & $\mathrm{Y}$ \\
\hline 254 & 184607.24 & -24220.7 & 29.92 & -0.05 & $99 \pm 14$ & $173 \pm 24$ & $116 \pm 16$ & $49 \pm 7$ & $16 \pm 3$ & $\mathrm{Y}$ \\
\hline 257 & 184606.94 & -24258.6 & 29.91 & -0.06 & $142 \pm 34$ & $97 \pm 24$ & $50 \pm 12$ & $25 \pm 6$ & $7.0 \pm 1.8$ & $\mathrm{Y}$ \\
\hline 258 & 184555.72 & -24231.1 & 29.89 & -0.01 & $177 \pm 22$ & $92 \pm 12$ & $48 \pm 6$ & $25 \pm 4$ & $11 \pm 2$ & Y \\
\hline 259 & 184607.95 & -24323.8 & 29.90 & -0.06 & $175 \pm 20$ & $78 \pm 10$ & $43 \pm 5$ & $18 \pm 3$ & $6.2 \pm 0.9$ & $\mathrm{Y}$ \\
\hline 262 & 184604.86 & -24244.1 & 29.91 & -0.05 & $81 \pm 10$ & $106 \pm 12$ & $58 \pm 7$ & $19 \pm 2$ & $6.5 \pm 0.9$ & $\mathrm{Y}$ \\
\hline 268 & 184545.64 & -23152.3 & 30.03 & +0.11 & $98 \pm 11$ & $48 \pm 5$ & $26 \pm 3$ & $13 \pm 2$ & $7.1 \pm 1.0$ & $\mathrm{Y}$ \\
\hline 269 & 184544.56 & -23218.4 & 30.02 & +0.11 & $215 \pm 24$ & $88 \pm 10$ & $41 \pm 5$ & $16 \pm 2$ & $4.5 \pm 0.6$ & Y \\
\hline 270 & 184609.73 & -24341.7 & 29.90 & -0.07 & $116 \pm 14$ & $60 \pm 7$ & $26 \pm 3$ & $10 \pm 1$ & $2.6 \pm 0.4$ & $\mathrm{~N}$ \\
\hline 274 & 184608.27 & -24804.0 & 29.83 & -0.10 & $104 \pm 12$ & $44 \pm 5$ & $26 \pm 3$ & $11 \pm 1$ & $4.2 \pm 0.6$ & $\mathrm{~N}$ \\
\hline
\end{tabular}


Table 1. continued.

\begin{tabular}{|c|c|c|c|c|c|c|c|c|c|c|}
\hline \# Id. ${ }^{a}$ & $\begin{array}{c}\alpha(\mathrm{J} 2000) \\
(\mathrm{h} \mathrm{m} \mathrm{s})\end{array}$ & $\begin{array}{c}\delta(\mathrm{J} 2000) \\
\left({ }^{\circ}{ }^{\prime \prime \prime}\right)\end{array}$ & $\begin{array}{c}l \\
\left({ }^{\circ}\right)\end{array}$ & $\begin{array}{c}b \\
\left({ }^{\circ}\right)\end{array}$ & $\begin{array}{c}S_{70 \mu \mathrm{m}} \\
(\mathrm{Jy})\end{array}$ & $\begin{array}{c}S_{160 \mu \mathrm{m}} \\
(\mathrm{Jy})\end{array}$ & $\begin{array}{c}S_{250 \mu \mathrm{m}} \\
(\mathrm{Jy})\end{array}$ & $\begin{array}{c}S_{350 \mu \mathrm{m}} \\
(\mathrm{Jy})\end{array}$ & $\begin{array}{c}S_{500 \mu \mathrm{m}} \\
(\mathrm{Jy})\end{array}$ & $24 \mu \mathrm{m}$ MIPS \\
\hline 275 & 184544.00 & -23200.3 & 30.03 & +0.11 & $123 \pm 14$ & $61 \pm 7$ & $30 \pm 3$ & $13 \pm 22$ & $4.1 \pm 0.6$ & $\mathrm{Y}$ \\
\hline 276 & 184608.37 & -24745.6 & 29.84 & -0.10 & $19 \pm 2$ & $16 \pm 2$ & $13 \pm 2$ & $9.0 \pm 1.2$ & $3.9 \pm 0.6$ & $\mathrm{Y}$ \\
\hline 278 & 184635.43 & -24034.6 & 30.00 & -0.14 & $33 \pm 4$ & $36 \pm 4$ & $26 \pm 3$ & $15 \pm 2$ & $6.5 \pm 0.9$ & Y \\
\hline 280 & 184626.29 & -24055.9 & 29.98 & -0.11 & $62 \pm 7$ & $46 \pm 5$ & $23 \pm 3$ & $8.9 \pm 1.2$ & $2.2 \pm 0.3$ & $\mathrm{Y}$ \\
\hline 281 & 184601.29 & -24623.4 & 29.85 & -0.06 & $63 \pm 7$ & $56 \pm 7$ & $36 \pm 4$ & $17 \pm 2$ & $5.0 \pm 0.7$ & Y \\
\hline 282 & 184551.24 & -2 3017.7 & 30.01 & +0.10 & $120 \pm 13$ & $50 \pm 6$ & $24 \pm 3$ & $9.5 \pm 1.2$ & $3.6 \pm 0.5$ & $\mathrm{Y}$ \\
\hline 285 & 184606.28 & -23013.5 & 30.10 & +0.04 & $24 \pm 3$ & $19 \pm 2$ & $11 \pm 1$ & $5.3 \pm 0.7$ & $2.2 \pm 0.3$ & $\mathrm{Y}$ \\
\hline 286 & 184559.66 & -22909.3 & 30.10 & +0.08 & $21 \pm 2$ & $32 \pm 4$ & $26 \pm 3$ & $14 \pm 2$ & $5.8 \pm 0.8$ & Y \\
\hline 288 & 184623.02 & -24305.6 & 29.94 & -0.12 & $46 \pm 5$ & $33 \pm 4$ & $17 \pm 2$ & $6.8 \pm 0.9$ & $2.4 \pm 0.3$ & $\mathrm{Y}$ \\
\hline 289 & 184622.69 & -24012.0 & 29.98 & -0.09 & $34 \pm 4$ & $34 \pm 4$ & $19 \pm 2$ & $8.2 \pm 1.1$ & $2.7 \pm 0.4$ & $\mathrm{Y}$ \\
\hline 291 & 184611.54 & -24415.3 & 29.90 & -0.08 & $26 \pm 3$ & $9.0 \pm 1.5$ & $4.0 \pm 0.8$ & $1.4 \pm 0.4$ & $0.14 \pm 0.12$ & $\mathrm{Y}$ \\
\hline 292 & 184614.13 & -24328.8 & 29.91 & -0.09 & $10 \pm 2$ & $9.4 \pm 1.9$ & $4.3 \pm 0.9$ & $1.9 \pm 0.4$ & $0.39 \pm 0.12$ & $\mathrm{Y}$ \\
\hline 293 & 184621.84 & -24030.7 & 29.97 & -0.09 & $35 \pm 4$ & $25 \pm 3$ & $13 \pm 1$ & $4.5 \pm 0.6$ & $0.95 \pm 0.17$ & $\mathrm{Y}$ \\
\hline 294 & 184625.27 & -24035.7 & 29.98 & -0.11 & $21 \pm 2$ & $18 \pm 2$ & $12 \pm 1$ & $5.9 \pm 0.8$ & $2.6 \pm 0.4$ & $\mathrm{Y}$ \\
\hline 301 & 184605.74 & -24828.1 & 29.82 & -0.09 & $30 \pm 3$ & $28 \pm 3$ & $22 \pm 2$ & $12 \pm 2$ & $4.7 \pm 0.7$ & $\mathrm{~N}$ \\
\hline 305 & 184617.13 & -24857.3 & 29.84 & -0.14 & $24 \pm 3$ & $17 \pm 2$ & $9.3 \pm 1.0$ & $4.3 \pm 0.6$ & $1.7 \pm 0.2$ & $\mathrm{Y}$ \\
\hline 306 & 184615.61 & -24921.4 & 29.83 & -0.14 & $12 \pm 1$ & $12 \pm 1$ & $6.8 \pm 0.8$ & $2.6 \pm 0.3$ & $0.70 \pm 0.10$ & $\mathrm{Y}$ \\
\hline 307 & 184622.52 & -24150.2 & 29.95 & -0.10 & $10 \pm 2$ & $8.3 \pm 1.8$ & $5.1 \pm 1.1$ & $2.3 \pm 0.5$ & $0.79 \pm 0.19$ & $\mathrm{Y}$ \\
\hline 309 & 184607.27 & -24857.0 & 29.82 & -0.10 & $16 \pm 2$ & $8.2 \pm 0.9$ & $4.5 \pm 0.6$ & $1.9 \pm 0.3$ & $1.0 \pm 0.1$ & $\mathrm{~N}$ \\
\hline 311 & 184603.84 & -23631.1 & 30.00 & +0.01 & $10 \pm 1$ & $11 \pm 1$ & $11 \pm 1$ & $7.1 \pm 0.9$ & $2.7 \pm 0.4$ & $\mathrm{Y}$ \\
\hline 318 & 184605.61 & -23517.5 & 30.02 & +0.01 & $12 \pm 1$ & $14 \pm 2$ & $11 \pm 1$ & $5.9 \pm 0.8$ & $1.7 \pm 0.2$ & $\mathrm{Y}$ \\
\hline 323 & 184603.43 & -23520.1 & 30.01 & +0.02 & $22 \pm 2$ & $15 \pm 2$ & $5.7 \pm 0.7$ & $1.1 \pm 0.2$ & $0.36 \pm 0.09$ & $\mathrm{Y}$ \\
\hline 324 & 184557.37 & -23650.2 & 29.98 & +0.03 & $21 \pm 3$ & $18 \pm 2$ & $8.2 \pm 0.9$ & $2.8 \pm 0.4$ & $0.53 \pm 0.10$ & $\mathrm{Y}$ \\
\hline 325 & 184556.12 & -24838.9 & 29.80 & -0.06 & $33 \pm 4$ & $24 \pm 3$ & $12 \pm 1$ & $4.6 \pm 0.6$ & $1.3 \pm 0.2$ & $\mathrm{Y}$ \\
\hline 326 & 184627.13 & -23948.3 & 29.99 & -0.11 & $6.3 \pm 0.8$ & $4.6 \pm 0.6$ & $2.6 \pm 0.4$ & $0.9 \pm 0.2$ & $0.08 \pm 0.02$ & $\mathrm{Y}$ \\
\hline 327 & 184619.36 & -24506.4 & 29.90 & -0.12 & $9.3 \pm 1.1$ & $6.1 \pm 0.7$ & $2.9 \pm 0.4$ & $1.4 \pm 0.2$ & $0.45 \pm 0.08$ & $\mathrm{Y}$ \\
\hline 329 & 184605.85 & -23033.3 & 30.09 & +0.04 & $25 \pm 3$ & $23 \pm 3$ & $12 \pm 1$ & $4.4 \pm 0.6$ & $1.1 \pm 0.2$ & $\mathrm{Y}$ \\
\hline 332 & 184621.60 & -25008.4 & 29.83 & -0.16 & $9.5 \pm 1.1$ & $4.4 \pm 0.5$ & $3.4 \pm 0.4$ & $1.5 \pm 0.2$ & $0.45 \pm 0.07$ & $\mathrm{Y}$ \\
\hline 335 & 184556.04 & -23747.5 & 29.96 & +0.02 & $8.7 \pm 1.2$ & $7.4 \pm 1.2$ & $3.3 \pm 0.6$ & $0.6 \pm 0.2$ & $0.08 \pm 0.02$ & $\mathrm{Y}$ \\
\hline 338 & 184649.17 & -23609.5 & 30.09 & -0.16 & $11 \pm 1$ & $14 \pm 2$ & $7.6 \pm 0.8$ & $3.2 \pm 0.4$ & $1.1 \pm 0.2$ & $\mathrm{Y}$ \\
\hline 339 & 184620.46 & -24634.1 & 29.88 & -0.13 & $7.9 \pm 0.9$ & $8.0 \pm 0.9$ & $4.9 \pm 0.6$ & $2.3 \pm 0.3$ & $1.2 \pm 0.2$ & $\mathrm{Y}$ \\
\hline 340 & 184640.22 & -23811.5 & 30.04 & -0.14 & $6.2 \pm 0.7$ & $8.8 \pm 1.0$ & $6.8 \pm 0.8$ & $3.5 \pm 0.5$ & $1.4 \pm 0.2$ & $\mathrm{Y}$ \\
\hline 341 & 184640.16 & -23534.1 & 30.08 & -0.12 & $13 \pm 1$ & $9.4 \pm 1.1$ & $3.2 \pm 0.4$ & $1.0 \pm 0.1$ & $0.31 \pm 0.05$ & $\mathrm{Y}$ \\
\hline 342 & 184542.77 & -23722.4 & 29.95 & +0.08 & $21 \pm 2$ & $17 \pm 2$ & $8.3 \pm 0.9$ & $3.2 \pm 0.4$ & $0.94 \pm 0.13$ & $\mathrm{Y}$ \\
\hline 343 & 184615.14 & -25114.3 & 29.80 & -0.15 & $5.9 \pm 0.7$ & $9.1 \pm 1.0$ & $6.6 \pm 0.7$ & $3.3 \pm 0.4$ & $1.2 \pm 0.17$ & $\mathrm{Y}$ \\
\hline 344 & 184625.04 & -24846.4 & 29.86 & -0.17 & $8.0 \pm 0.9$ & $5.1 \pm 0.6$ & $2.6 \pm 0.3$ & $1.2 \pm 0.2$ & $0.51 \pm 0.07$ & Y \\
\hline 349 & 184649.98 & -24249.3 & 29.99 & -0.21 & $13 \pm 1$ & $4.7 \pm 0.5$ & $2.0 \pm 0.3$ & $0.96 \pm 0.15$ & $0.35 \pm 0.06$ & $\mathrm{Y}$ \\
\hline 352 & 184613.30 & -23235.8 & 30.07 & +0.00 & $2.4 \pm 0.3$ & $4.4 \pm 0.5$ & $3.3 \pm 0.4$ & $1.6 \pm 0.2$ & $0.46 \pm 0.06$ & $\mathrm{Y}$ \\
\hline 357 & 184558.60 & -23502.0 & 30.01 & +0.04 & $0.31 \pm 0.09$ & $2.8 \pm 0.7$ & $5.9 \pm 1.3$ & $5.5 \pm 1.2$ & $2.9 \pm 0.7$ & $\mathrm{Y}$ \\
\hline
\end{tabular}


Table 2. Mid-infrared fluxes used for the SED fitting.

\begin{tabular}{llcc}
\hline \hline & & $\lambda$ & $S$ \\
\# Id. $^{a}$ & Instrument & $\begin{array}{c}\lambda \mathrm{m}) \\
(\mathrm{Jy})\end{array}$ \\
\hline 132 & MIPSGAL & 24 & 0.44 \\
135 & MIPSGAL & 24 & 0.27 \\
143 & MIPSGAL & 24 & 0.024 \\
147 & MIPSGAL & 24 & 0.047 \\
242 & MSX & 21 & 1340 \\
243 & MSX & 21 & 19 \\
245 & MSX & 21 & 26 \\
247 & WISE & 22 & 16 \\
251 & MIPSGAL & 24 & 4.6 \\
253 & WISE & 22 & 9.9 \\
257 & WISE & 22 & 4.3 \\
258 & MIPSGAL & 24 & 1.5 \\
259 & MIPSGAL & 24 & 1.2 \\
268 & MIPSGAL & 24 & 2.0 \\
269 & MIPSGAL & 24 & 3.9 \\
270 & WISE & 22 & 1.3 \\
274 & WISE & 22 & 2.7 \\
275 & MIPSGAL & 24 & 1.02 \\
282 & MIPSGAL & 24 & 1.1 \\
285 & MIPSGAL & 24 & 0.74 \\
288 & MIPSGAL & 24 & 0.52 \\
291 & MIPSGAL & 24 & 0.021 \\
305 & MIPSGAL & 24 & 0.31 \\
309 & WISE & 22 & 0.49 \\
327 & MIPSGAL & 24 & 0.20 \\
342 & MIPSGAL & 24 & 0.16 \\
344 & MSX & 21 & 8.0 \\
349 & MIPSGAL & 24 & 0.39 \\
\hline & & & \\
& &
\end{tabular}

Notes. ${ }^{(a)}$ The number corresponds to the Hi-GAL identification number (Table 1).
Table 3. Properties of the Hi-GAL sources in the G29.96-0.02 cloud from the SED fitting.

\begin{tabular}{|c|c|c|c|c|c|c|}
\hline \# Id. & $\beta$ & $\begin{array}{c}\theta \\
\left({ }^{\prime \prime}\right)\end{array}$ & $\begin{array}{c}T \\
(\mathrm{~K})\end{array}$ & $\begin{array}{l}M_{\text {env }} \\
\left(M_{\odot}\right)\end{array}$ & $\begin{array}{c}\Sigma \\
\left(\mathrm{g} \mathrm{cm}^{-2}\right)\end{array}$ & $\begin{array}{l}L_{\text {bol }} \\
\left(L_{\odot}\right)\end{array}$ \\
\hline 1 & 0.8 & 19.44 & 41.9 & 645 & 0.50 & 3823 \\
\hline 2 & 1.4 & 27.48 & 27.4 & 966 & 0.38 & 7577 \\
\hline 4 & 1.2 & 21.81 & 15.8 & 2426 & 1.5 & 705 \\
\hline 5 & 2.2 & 18.75 & 15.8 & 216 & 0.18 & 438 \\
\hline 6 & 1.8 & 18.30 & 15.8 & 457 & 0.40 & 427 \\
\hline 7 & 2.2 & 17.67 & 15.8 & 172 & 0.16 & 363 \\
\hline 8 & 1.4 & 18.91 & 18.7 & 257 & 0.21 & 272 \\
\hline 9 & 2.0 & 18.19 & 12.9 & 272 & 0.24 & 111 \\
\hline 11 & 1.8 & 23.06 & 21.6 & 41 & 0.02 & 233 \\
\hline 12 & 2.6 & 22.26 & 10.0 & 513 & 0.31 & 123 \\
\hline 13 & 1.0 & 25.56 & 18.7 & 543 & 0.25 & 253 \\
\hline 14 & 0.8 & 26.24 & 27.4 & 204 & 0.09 & 400 \\
\hline 16 & 0.6 & 23.91 & 24.5 & 323 & 0.17 & 188 \\
\hline 17 & 0.4 & 27.48 & 27.4 & 724 & 0.28 & 405 \\
\hline 18 & 1.2 & 17.28 & 15.8 & 257 & 0.25 & 75 \\
\hline 19 & 1.6 & 24.07 & 18.7 & 115 & 0.06 & 185 \\
\hline 20 & 1.8 & 26.33 & 12.9 & 363 & 0.15 & 103 \\
\hline 21 & 1.8 & 27.48 & 18.7 & 91 & 0.04 & 226 \\
\hline 22 & 1.6 & 26.38 & 21.6 & 68 & 0.03 & 247 \\
\hline 24 & 1.8 & 24.39 & 18.7 & 72 & 0.04 & 180 \\
\hline 25 & 1.4 & 26.15 & 21.6 & 172 & 0.07 & 197 \\
\hline 26 & 2.0 & 24.27 & 12.9 & 257 & 0.13 & 105 \\
\hline 27 & 1.2 & 23.59 & 24.5 & 122 & 0.06 & 232 \\
\hline 31 & 2.6 & 21.48 & 10.0 & 431 & 0.28 & 104 \\
\hline 33 & 1.8 & 23.80 & 21.6 & 48 & 0.03 & 277 \\
\hline 34 & 1.6 & 26.87 & 18.7 & 108 & 0.04 & 175 \\
\hline 35 & 1.8 & 24.00 & 24.5 & 36 & 0.02 & 243 \\
\hline 37 & 1.2 & 19.97 & 21.6 & 129 & 0.10 & 190 \\
\hline 38 & 1.4 & 23.16 & 24.5 & 46 & 0.03 & 208 \\
\hline 40 & 1.2 & 18.57 & 27.4 & 54 & 0.05 & 222 \\
\hline 42 & 1.4 & 26.38 & 24.5 & 46 & 0.02 & 208 \\
\hline 44 & 0.4 & 25.37 & 33.2 & 172 & 0.08 & 315 \\
\hline 45 & 1.2 & 23.28 & 21.6 & 243 & 0.13 & 193 \\
\hline 48 & 1.4 & 22.49 & 27.4 & 32 & 0.02 & 269 \\
\hline 49 & 1.0 & 20.18 & 27.4 & 77 & 0.06 & 201 \\
\hline 54 & 2.4 & 22.40 & 21.6 & 7.7 & 0.005 & 183 \\
\hline 55 & 2.4 & 21.59 & 12.9 & 108 & 0.07 & 96 \\
\hline 61 & 2.4 & 27.20 & 15.8 & 54 & 0.02 & 176 \\
\hline 62 & 0.6 & 23.19 & 27.4 & 122 & 0.07 & 136 \\
\hline 64 & 1.0 & 19.60 & 24.5 & 122 & 0.09 & 170 \\
\hline 65 & 1.4 & 24.80 & 24.5 & 54 & 0.03 & 247 \\
\hline 66 & 0.8 & 24.30 & 27.4 & 91 & 0.05 & 158 \\
\hline 69 & 1.2 & 25.05 & 30.3 & 58 & 0.03 & 494 \\
\hline 70 & 2.4 & 24.43 & 10.0 & 645 & 0.32 & 111 \\
\hline 73 & 2.6 & 24.41 & 10.0 & 305 & 0.15 & 70 \\
\hline 74 & 2.2 & 24.87 & 15.8 & 72 & 0.03 & 152 \\
\hline 75 & 2.6 & 23.23 & 15.8 & 23 & 0.01 & 114 \\
\hline 77 & 1.0 & 21.61 & 27.4 & 58 & 0.04 & 181 \\
\hline 79 & 2.0 & 27.48 & 24.5 & 32 & 0.01 & 287 \\
\hline 81 & 0.8 & 21.63 & 30.3 & 77 & 0.05 & 244 \\
\hline 83 & 2.4 & 23.39 & 12.9 & 77 & 0.04 & 67 \\
\hline 86 & 2.2 & 12.81 & 15.8 & 27 & 0.05 & 58 \\
\hline 87 & 1.6 & 25.50 & 21.6 & 38 & 0.02 & 139 \\
\hline
\end{tabular}


Table 3. continued.

\begin{tabular}{|c|c|c|c|c|c|c|}
\hline \# Id. & $\beta$ & $\begin{array}{c}\theta \\
\left({ }^{\prime \prime}\right)\end{array}$ & $\begin{array}{c}T \\
(\mathrm{~K}) \\
\end{array}$ & $\begin{array}{l}M_{\text {env }} \\
\left(M_{\odot}\right)\end{array}$ & $\begin{array}{c}\Sigma \\
\left(\mathrm{g} \mathrm{cm}^{-2}\right) \\
\end{array}$ & $\begin{array}{l}L_{\text {bol }} \\
\left(L_{\odot}\right) \\
\end{array}$ \\
\hline 88 & 2.0 & 27.48 & 18.7 & 48 & 0.02 & 187 \\
\hline 90 & 1.2 & 27.11 & 24.5 & 61 & 0.02 & 173 \\
\hline 98 & 0.8 & 27.48 & 24.5 & 513 & 0.20 & 336 \\
\hline 99 & 0.4 & 22.96 & 33.2 & 108 & 0.06 & 199 \\
\hline 100 & 2.4 & 16.04 & 24.5 & 3.8 & 0.004 & 206 \\
\hline 104 & 2.0 & 25.49 & 18.7 & 41 & 0.02 & 157 \\
\hline 109 & 1.6 & 19.07 & 21.6 & 43 & 0.04 & 156 \\
\hline 111 & 1.2 & 21.09 & 21.6 & 86 & 0.06 & 127 \\
\hline 113 & 2.0 & 19.03 & 12.9 & 122 & 0.10 & 50 \\
\hline 122 & 1.8 & 26.27 & 27.4 & 575 & 0.25 & 12985 \\
\hline 123 & 1.4 & 24.86 & 21.6 & 3234 & 1.54 & 7446 \\
\hline 124 & 2.6 & 13.86 & 39.0 & 102 & 0.16 & 3544 \\
\hline 125 & 0.6 & 23.61 & 41.9 & 1288 & 0.68 & 11232 \\
\hline 126 & 0.6 & 19.55 & 27.4 & 3844 & 3.0 & 4647 \\
\hline 127 & 1.4 & 28.26 & 30.3 & 645 & 0.24 & 8845 \\
\hline 129 & 0.4 & 21.03 & 30.3 & 2161 & 1.4 & 2651 \\
\hline 130 & 2.2 & 26.26 & 12.9 & 2883 & 1.2 & 1739 \\
\hline 131 & 1.4 & 20.69 & 15.8 & 2041 & 1.4 & 821 \\
\hline 132 & 1.2 & 24.30 & 41.9 & 81 & 0.04 & 3765 \\
\hline 133 & 1.8 & 19.34 & 15.8 & 767 & 0.60 & 677 \\
\hline 134 & 1.2 & 21.90 & 15.8 & 2883 & 1.8 & 798 \\
\hline 135 & 2.0 & 18.32 & 39.0 & 4.3 & 0.004 & 1369 \\
\hline 136 & 1.8 & 21.60 & 15.8 & 363 & 0.23 & 339 \\
\hline 137 & 1.8 & 28.28 & 21.6 & 431 & 0.16 & 2338 \\
\hline 138 & 2.2 & 21.19 & 21.6 & 48 & 0.03 & 713 \\
\hline 139 & 1.4 & 24.27 & 36.1 & 86 & 0.04 & 1555 \\
\hline 141 & 2.4 & 12.37 & 24.5 & 6.5 & 0.01 & 345 \\
\hline 142 & 2.0 & 27.48 & 15.8 & 343 & 0.13 & 461 \\
\hline 143 & 1.0 & 14.80 & 44.8 & 3.8 & 0.005 & 140 \\
\hline 144 & 0.6 & 26.62 & 39.0 & 115 & 0.05 & 720 \\
\hline 147 & 1.6 & 24.34 & 36.1 & 16 & 0.008 & 1041 \\
\hline 148 & 1.4 & 26.16 & 18.7 & 767 & 0.33 & 811 \\
\hline 149 & 1.8 & 22.43 & 18.7 & 384 & 0.23 & 919 \\
\hline 150 & 2.2 & 25.70 & 15.8 & 431 & 0.19 & 915 \\
\hline 151 & 1.6 & 28.24 & 21.6 & 609 & 0.23 & 312 \\
\hline 152 & 2.2 & 30.00 & 18.7 & 162 & 0.05 & 930 \\
\hline 153 & 1.6 & 30.00 & 36.1 & 51 & 0.02 & 1528 \\
\hline 155 & 0.4 & 24.92 & 24.5 & 1147 & 0.54 & 337 \\
\hline 159 & 1.4 & 26.74 & 18.7 & 861 & 0.35 & 885 \\
\hline 160 & 2.6 & 25.77 & 30.3 & 12 & 0.005 & 894 \\
\hline 161 & 2.6 & 23.14 & 21.6 & 32 & 0.02 & 237 \\
\hline 162 & 1.6 & 24.09 & 21.6 & 243 & 0.12 & 258 \\
\hline 163 & 2.0 & 16.97 & 21.6 & 136 & 0.14 & 265 \\
\hline 164 & 0.6 & 17.39 & 21.6 & 484 & 0.47 & 200 \\
\hline 165 & 0.8 & 24.47 & 30.3 & 272 & 0.13 & 594 \\
\hline 167 & 1.6 & 23.25 & 27.4 & 51 & 0.03 & 702 \\
\hline 168 & 1.4 & 30.00 & 21.6 & 288 & 0.09 & 661 \\
\hline 169 & 0.4 & 17.80 & 33.2 & 243 & 0.23 & 445 \\
\hline 170 & 1.4 & 25.20 & 27.4 & 153 & 0.07 & 563 \\
\hline 171 & 1.6 & 21.54 & 15.8 & 645 & 0.41 & 405 \\
\hline 172 & 0.4 & 25.67 & 27.4 & 5432 & 2.4 & 860 \\
\hline 175 & 0.4 & 17.39 & 21.6 & 2426 & 2.4 & 279 \\
\hline 177 & 1.0 & 30.00 & 27.4 & 323 & 0.11 & 1017 \\
\hline 178 & 1.4 & 25.93 & 21.6 & 243 & 0.11 & 559 \\
\hline
\end{tabular}

Table 3. continued.

\begin{tabular}{|c|c|c|c|c|c|c|}
\hline \# Id. & $\beta$ & $\begin{array}{c}\theta \\
\left({ }^{\prime \prime}\right)\end{array}$ & $\begin{array}{c}T \\
(\mathrm{~K})\end{array}$ & $\begin{array}{l}M_{\text {env }} \\
\left(M_{\odot}\right)\end{array}$ & $\begin{array}{c}\Sigma \\
\left(\mathrm{g} \mathrm{cm}^{-2}\right)\end{array}$ & $\begin{array}{l}L_{\text {bol }} \\
\left(L_{\odot}\right)\end{array}$ \\
\hline 179 & 1.0 & 22.50 & 36.1 & 38 & 0.02 & 425 \\
\hline 180 & 1.4 & 22.70 & 30.3 & 72 & 0.04 & 617 \\
\hline 182 & 2.2 & 29.25 & 15.8 & 323 & 0.11 & 686 \\
\hline 184 & 0.4 & 28.02 & 24.5 & 2426 & 0.91 & 469 \\
\hline 185 & 1.8 & 24.95 & 24.5 & 31 & 0.01 & 363 \\
\hline 186 & 1.8 & 27.68 & 27.4 & 27 & 0.01 & 362 \\
\hline 187 & 1.8 & 30.00 & 27.4 & 97 & 0.03 & 639 \\
\hline 188 & 1.4 & 24.14 & 24.5 & 48 & 0.02 & 220 \\
\hline 189 & 0.6 & 24.57 & 36.1 & 108 & 0.05 & 476 \\
\hline 191 & 1.8 & 25.75 & 21.6 & 81 & 0.04 & 466 \\
\hline 192 & 2.0 & 24.94 & 18.7 & 61 & 0.03 & 234 \\
\hline 193 & 2.2 & 16.71 & 21.6 & 288 & 0.30 & 314 \\
\hline 194 & 0.6 & 28.03 & 39.0 & 216 & 0.08 & 1094 \\
\hline 195 & 0.6 & 18.93 & 18.7 & 861 & 0.71 & 184 \\
\hline 196 & 1.4 & 30.00 & 21.6 & 204 & 0.07 & 470 \\
\hline 197 & 2.6 & 27.99 & 30.3 & 6 & 0.002 & 724 \\
\hline 198 & 2.0 & 28.68 & 24.5 & 34 & 0.01 & 668 \\
\hline 199 & 2.0 & 29.81 & 24.5 & 46 & 0.02 & 891 \\
\hline 200 & 2.2 & 26.78 & 21.6 & 91 & 0.04 & 260 \\
\hline 201 & 1.4 & 30.00 & 30.3 & 41 & 0.01 & 422 \\
\hline 202 & 2.0 & 24.03 & 21.6 & 27 & 0.01 & 249 \\
\hline 203 & 1.0 & 25.12 & 21.6 & 384 & 0.18 & 368 \\
\hline 205 & 1.4 & 30.00 & 30.3 & 68 & 0.02 & 596 \\
\hline 207 & 1.4 & 25.40 & 36.1 & 24 & 0.01 & 694 \\
\hline 208 & 2.2 & 22.35 & 18.7 & 29 & 0.02 & 173 \\
\hline 209 & 0.4 & 23.35 & 18.7 & 2883 & 1.6 & 423 \\
\hline 210 & 2.0 & 29.79 & 27.4 & 16 & 0.005 & 618 \\
\hline 212 & 0.8 & 17.82 & 21.6 & 543 & 0.50 & 340 \\
\hline 213 & 1.8 & 29.56 & 24.5 & 43 & 0.02 & 513 \\
\hline 214 & 2.6 & 29.64 & 12.9 & 407 & 0.14 & 456 \\
\hline 215 & 0.8 & 25.20 & 21.6 & 431 & 0.20 & 270 \\
\hline 217 & 2.4 & 30.00 & 15.8 & 102 & 0.03 & 331 \\
\hline 219 & 2.4 & 28.04 & 15.8 & 48 & 0.02 & 154 \\
\hline 220 & 1.4 & 30.00 & 24.5 & 86 & 0.03 & 391 \\
\hline 221 & 2.0 & 28.99 & 21.6 & 48 & 0.02 & 441 \\
\hline 228 & 2.2 & 27.80 & 15.8 & 129 & 0.05 & 267 \\
\hline 232 & 1.6 & 30.00 & 24.5 & 108 & 0.04 & 344 \\
\hline 242 & 0.8 & 19.39 & 76.7 & 2883 & 2.3 & 791095 \\
\hline 243 & 1.4 & 14.84 & 70.9 & 431 & 0.58 & 39363 \\
\hline 245 & 2.6 & 23.75 & 73.8 & 229 & 0.12 & 47243 \\
\hline 247 & 0.6 & 23.65 & 56.4 & 1215 & 0.64 & 41592 \\
\hline 251 & 1.6 & 21.38 & 56.4 & 72 & 0.05 & 13062 \\
\hline 253 & 1.8 & 27.42 & 62.2 & 193 & 0.08 & 23156 \\
\hline 254 & 1.4 & 22.55 & 27.4 & 1023 & 0.59 & 7999 \\
\hline 257 & 0.6 & 19.57 & 59.3 & 457 & 0.35 & 11617 \\
\hline 258 & 0.8 & 21.06 & 44.8 & 513 & 0.34 & 10648 \\
\hline 259 & 1.2 & 23.91 & 41.9 & 229 & 0.12 & 10559 \\
\hline 262 & 2.4 & 22.82 & 44.8 & 77 & 0.04 & 6097 \\
\hline 268 & 0.4 & 16.48 & 53.5 & 384 & 0.42 & 5751 \\
\hline 269 & 1.4 & 26.91 & 44.8 & 129 & 0.05 & 15111 \\
\hline 270 & 1.4 & 25.58 & 53.5 & 72 & 0.03 & 8826 \\
\hline 274 & 0.6 & 25.40 & 56.4 & 216 & 0.10 & 7396 \\
\hline 275 & 1.2 & 29.45 & 41.9 & 162 & 0.06 & 7511 \\
\hline 276 & 0.4 & 11.75 & 39.0 & 323 & 0.69 & 1204 \\
\hline
\end{tabular}


M. T. Beltrán et al.: Hi-GAL sources in the G29.96-0.02 cloud

Table 3. continued.

\begin{tabular}{ccccccc}
\hline \hline & & $\theta$ & $T$ & $M_{\text {env }}$ & $\Sigma$ & $\begin{array}{c}L_{\text {bol }} \\
\left(\mathrm{g} \mathrm{cm}^{-2}\right)\end{array}$ \\
\# Id. & $\beta$ & $\left({ }_{\odot}\right)$ & $(\mathrm{K})$ & $\left(M_{\odot}\right)$ & 2268 \\
\hline 278 & 0.4 & 13.71 & 39.0 & 609 & 0.96 & 3690 \\
280 & 1.8 & 22.85 & 41.9 & 54 & 0.03 & 3698 \\
281 & 1.4 & 27.87 & 41.9 & 204 & 0.08 & 4078 \\
282 & 1.0 & 27.69 & 44.8 & 162 & 0.06 & 5954 \\
285 & 0.4 & 15.01 & 53.5 & 144 & 0.19 & 1966 \\
286 & 0.6 & 14.34 & 36.1 & 645 & 0.92 & 1637 \\
288 & 0.8 & 23.29 & 47.7 & 144 & 0.08 & 3018 \\
289 & 1.2 & 26.84 & 39.0 & 136 & 0.06 & 2195 \\
291 & 2.6 & 24.19 & 30.3 & 3.8 & 0.002 & 1390 \\
292 & 2.0 & 18.77 & 27.4 & 15 & 0.01 & 584 \\
293 & 2.2 & 27.86 & 27.4 & 31 & 0.01 & 1965 \\
294 & 0.6 & 23.29 & 41.9 & 193 & 0.11 & 1375 \\
301 & 0.4 & 30.00 & 41.9 & 513 & 0.17 & 1957 \\
305 & 0.6 & 30.00 & 47.7 & 102 & 0.03 & 1619 \\
306 & 1.6 & 23.90 & 30.3 & 32 & 0.02 & 779 \\
307 & 1.0 & 21.63 & 36.1 & 48 & 0.03 & 604 \\
309 & 0.4 & 24.86 & 59.3 & 51 & 0.02 & 1206 \\
311 & 0.4 & 13.35 & 36.1 & 323 & 0.53 & 717 \\
318 & 0.8 & 15.52 & 36.1 & 153 & 0.19 & 875 \\
323 & 2.6 & 27.19 & 27.4 & 6.5 & 0.003 & 1195 \\
324 & 2.6 & 24.65 & 24.5 & 14 & 0.007 & 1224 \\
325 & 1.6 & 27.05 & 39.0 & 41 & 0.02 & 1978 \\
326 & 2.6 & 24.05 & 24.5 & 3.8 & 0.002 & 344 \\
327 & 0.6 & 22.58 & 50.6 & 31 & 0.02 & 634 \\
329 & 2.0 & 26.47 & 27.4 & 41 & 0.02 & 1554 \\
332 & 0.8 & 26.97 & 44.8 & 27 & 0.01 & 565 \\
335 & 2.4 & 26.01 & 27.4 & 3.8 & 0.002 & 421 \\
338 & 1.4 & 30.00 & 33.2 & 51 & 0.02 & 778 \\
339 & 0.6 & 25.09 & 39.0 & 81 & 0.04 & 509 \\
340 & 0.8 & 21.85 & 33.2 & 115 & 0.07 & 471 \\
341 & 2.2 & 30.00 & 33.2 & 5.8 & 0.002 & 688 \\
342 & 1.0 & 25.91 & 41.9 & 58 & 0.03 & 1512 \\
343 & 1.0 & 26.35 & 30.3 & 91 & 0.04 & 474 \\
344 & 0.8 & 25.27 & 44.8 & 26 & 0.01 & 505 \\
349 & 1.0 & 30.00 & 50.6 & 12 & 0.004 & 821 \\
352 & 1.4 & 17.62 & 27.4 & 32 & 0.03 & 213 \\
357 & 0.4 & 9.66 & 21.6 & 683 & 2.2 & 140 \\
\hline & & & & & &
\end{tabular}

